\title{
A review on the green and sustainable synthesis of silver nanoparticles and one-dimensional silver nanostructures
}

\author{
Sina Kaabipour and Shohreh Hemmati ${ }^{\star}$
}

\author{
Review \\ Address: \\ School of Chemical Engineering, Oklahoma State University, \\ Stillwater, Oklahoma, 74078, USA \\ Email: \\ Sina Kaabipour - skaabip@okstate.edu; \\ Shohreh Hemmati ${ }^{*}$ - shohreh.hemmati@okstate.edu \\ * Corresponding author \\ Keywords: \\ green nanotechnology; green reagents; green synthesis; \\ one-dimensional silver nanostructure; silver nanostructures
}

Beilstein J. Nanotechnol. 2021, 12, 102-136.

https://doi.org/10.3762/bjnano.12.9

Received: 01 September 2020

Accepted: 14 December 2020

Published: 25 January 2021

Associate Editor: C. T. Yavuz

(C) 2021 Kaabipour and Hemmati; licensee Beilstein-Institut. License and terms: see end of document.

\begin{abstract}
The significance of silver nanostructures has been growing considerably, thanks to their ubiquitous presence in numerous applications, including but not limited to renewable energy, electronics, biosensors, wastewater treatment, medicine, and clinical equipment. The properties of silver nanostructures, such as size, size distribution, and morphology, are strongly dependent on synthesis process conditions such as the process type, equipment type, reagent type, precursor concentration, temperature, process duration, and $\mathrm{pH}$. Physical and chemical methods have been among the most common methods to synthesize silver nanostructures; however, they possess substantial disadvantages and short-comings, especially compared to green synthesis methods. On the contrary, the number of green synthesis techniques has been increasing during the last decade and they have emerged as alternative routes towards facile and effective synthesis of silver nanostructures with different morphologies. In this review, we have initially outlined the most common and popular chemical and physical methodologies and reviewed their advantages and disadvantages. Green synthesis methodologies are then discussed in detail and their advantages over chemical and physical methods have been noted. Recent studies are then reviewed in detail and the effects of essential reaction parameters, such as temperature, $\mathrm{pH}$, precursor, and reagent concentration, on silver nanostructure size and morphology are discussed. Also, green synthesis techniques used for the synthesis of one-dimensional (1D) silver nanostructures have been reviewed, and the potential of alternative green reagents for their synthesis has been discussed. Furthermore, current challenges regarding the green synthesis of 1D silver nanostructures and future direction are outlined. To sum up, we aim to show the real potential of green nanotechnology towards the synthesis of silver nanostructures with various morphologies (especially $1 \mathrm{D}$ ones) and the possibility of altering current techniques towards more environmentally friendly, more energy-efficient, less hazardous, simpler, and cheaper procedures.
\end{abstract}




\section{Review}

\section{Introduction}

Nanotechnology has been ubiquitously applied in almost every scientific discipline. Nanomaterials have been utilized in innumerable applications due to their unique characteristics. Novel, successful applications of nanomaterials and nanostructures can be seen in drug delivery [1-6], nanomedicine [7-10], food packaging [11-13], aseptic procedures [14-16], correlative microscopy [17], imaging [18-22], optics [23,24], microelectronics [2527], three dimensional (3D) printing [27-31], renewable energy [32-36], wastewater remediation [37,38], and catalysis [39-43], to name a few. The success of nanotechnology has been established and the promising outcomes cannot be overlooked; however, the main principles behind the production of nanomaterials are yet to be examined more closely in terms of economy as well as effects on health and environment.

Among metal nanostructures, silver nanostructures have demonstrated promising potential in many applications and have contributed significantly to the advancement of nanoscience. The concept of using silver is not unprecedented; silver was used broadly by many nations and dynasties throughout ancient history. The early applications of silver date back to 4000 B.C.E, by the Caldeans [44], and throughout ancient history, Persians, Romans, Egyptians, and Greeks utilized silver for food storage purposes [45]. Silver was also extensively used in utensils for eating and drinking, most probably due to the antimicrobial activities of silver which were discovered from experience during ancient centuries [45]. Later on, there were further instances where silver was used for medical purposes. For instance, Avi-Cenna applied silver filings for blood purification and to inhibit heart palpitations [44]. Silver was used in forms of aqueous solutions, coins, and plates for aseptic and antibacterial applications, as well as in food and dairy preservation up until the 19th century, as it was agreed that food and dairy products kept in silver containers lasted longer than those in other types of containers [44]. Since then, silver has been and is still in use in accordance with these old traditions even today. Although silver has proved its potential and advantages, the mechanisms behind its functionality were not well understood in the past. Nanotechnology has emerged as a means to delve further into the usefulness of this precious element.

Silver nanostructures can be categorized based on their shape and morphology. Different shapes and morphologies of silver nanostructures have been synthesized, including cubes [46,47], spheres [48-50], triangles [51-53], prisms [54,55], sheets [56$58]$, disks $[59,60]$, rods $[61,62]$, bars [63,64], and wires [65-68]

Silver nanostructures have received significant attention in the last decades due to their improved properties compared to bulk silver and their unique intrinsic characteristics including antimicrobial activity, electrical conductivity, thermal conductivity, optical characteristics, and mechanical properties. The antimicrobial characteristic of silver nanoparticles (AgNPs) has made them highly applicable in the biomedical and therapeutic fields [69-71]. Currently, antimicrobial AgNPs can potentially act as alternatives for current antibiotics due to increased bacterial resistance [71]. In addition, the development of new generations of antibiotics is costly, which prevents pharmaceutical companies from manufacturing new classes of antibiotics [72,73]. Furthermore, the physicochemical characteristics of AgNPs can be tuned in a way to avoid cellular toxicity [71,74,75], which facilitates their biomedical applications. The small size of AgNPs $(<100 \mathrm{~nm})$ allows them to accumulate on the extracellular membrane of the bacteria and penetrate inside, which alters the membrane permeability and leads to bacterial death $[71,76]$. Another therapeutic approach lies in the bactericidal activity of functionalized silver nanoparticles coated on surfaces. This method is applicable in developing aseptic catheters to prevent catheter-related infections such as urinary tract and venous infections, and also inhibit the growth of bacterial biofilms [14-16].

AgNPs were also used in developing strong thermally conductive materials. They were used in polymer composites to increase thermal conductivity $(\mathrm{K})[77,78]$ for cooling applications in electronic equipment. Furthermore, AgNPs have demonstrated unique electrical properties. AgNPs coated on polycarbonate substrates were previously used to increase the electrical conductivity of polycarbonate composites [79]. AgNPs have also demonstrated minimum or no adverse effects on mechanical strength when embedded in polymeric materials or composites [80-82]. For instance, the utilization of AgNPs in bone cement is meant to prevent bacterial infection while sustaining the mechanical strength of the cement connected to the prosthesis [82]. AgNPs have also demonstrated significant optical properties. They possess substantial surface plasmon resonance (SPR) and generally have a broad absorption spectrum [83]. This enables applications in optoelectronics and surface-enhanced Raman scattering [84,85]. AgNPs were also applied effectively in solar cell matrices [32,86,87]. AgNPs can enhance the current density in solar cells due to their far-field effect and localized surface plasmon resonance (LSPR) [32].

There are several applications in which use of 1D silver nanostructures such as nanowires (NWs) and nanorods (NRs) (at the same concentration) are preferred to other nanostructures due to stronger conductivity. For instance, 1D silver nanostructures can provide desired electrical characteristics in conductive 
adhesives at lower concentrations compared to other silver nanostructures and micrometer-sized ones [88]. The research being conducted on the synthesis of silver nanowires (AgNWs) is currently gaining a lot of attention due to their promising applications in electronics. This is a result of the outstanding electrical, optical, and mechanical properties of AgNWs. 1D silver nanostructures, such as AgNWs are more advantageous compared to other silver nanostructures due to several reasons. They can enable free movement of electrons in one direction [89], and can form networks of wires that facilitate the passage of electrical current. They can also improve transmittance characteristics due to their high aspect ratio [90]. Novel applications can be seen in transparent conductive films (TCFs) [91], wireless technology [92,93], touchscreen devices [94], organic light emitting diodes (OLED) [95], transparent conductive electrodes [96,97], artificial skin [98], liquid crystal display (LCD) $[99,100]$, and smart windows $[101,102]$. AgNWs can be embedded in flexible touch-screen substrates and electronic displays to provide an enhanced decrease in sheet resistance and to increase touch sensitivity [89]. Furthermore, AgNWs can be used to prepare AgNW-based conductive inks that have remarkable rheological characteristics such as thixotropic shear thinning and thus, can be simply used for screen printing without the addition of polymeric rheological additives [103]. AgNW-coated conductive films have been considered as a promising alternative over conventional indium tin oxide (ITO)-coated conductive films [89].

The synthesis of silver nanostructures, and generally all types of nanostructures, can be categorized as one of two approaches [104]. The first one is the top-down approach where particles are produced from the bulk material, and the second one is the bottom-up approach where nucleation sites are formed and finally grow into a nanometer-sized particle. The first approach consists of a set of techniques also known as "physical" synthesis methods. Several physical methods for top-down synthesis of silver nanostructures including ball milling [105-107], laser ablation [108-112], evaporation-condensation [113,114], electromagnetic levitation gas condensation (ELGC) [115], ultrasonication [116-119], lithography [120,121], spray pyrolysis [122-124], radiolysis [125-128], arc discharge [129-133], and photoirradiation [134-136] have been utilized to synthesize various morphologies of silver nanostructures with varied size and size distribution. The physical synthesis method is primarily used for large-scale production in a short amount of time [137].

The bottom-up approach, however, mostly relies on the use of reducing agents for the production of silver nanoparticles. This approach is also categorized into two distinguishable, but not completely disparate, set of methods. The first category consists of techniques that use chemical reagents to reduce $\mathrm{Ag}$ cations into zero-charged Ag atoms, which then mount on top of the nuclei, serving as templates for crystal growth into particles at the nanoscale $[138,139]$. This set of techniques is also known as the "chemical" synthesis method. These techniques are usually accompanied by the addition of stabilizers to provide stability, prevent aggregation, control morphology, and provide physiologically compatible properties [140-142]. Chemical methods were previously used to produce silver nanoparticles including sol-gel processes [143-146], conventional chemical reduction [147-151], reverse micelle [152-154], co-precipitation [155], chemical vapor deposition [156-158], solvothermal [159-161], and electrochemical reduction [162-165]. Chemical synthesis methods are currently among the most widely used approaches $[104,166]$. The second category in bottom-up synthesis methods consists of a set of techniques that incorporate the use of nonchemical reagents for the synthesis of silver nanostructures. Those techniques rely on the use of biological agents or bioextracted compounds. The term used for addressing these methods is also referred to as "biological" synthesis. Previous studies have used bacteria [167-170], fungi [171-174], viruses [175,176], yeasts [177-179], plants [180-183] and plant extracts [166,184-188], microalgae [189-193], enzymes [194-196], saccharides [197-201], and vitamins [202-204] to synthesize Ag nanostructures with controlled size and morphology. These methods can also be referred to as a subcategory of green synthesis.

Numerous works in the literature have focused on synthesis of AgNPs without giving much attention to the disadvantages such as cost, time, and hazards. Nevertheless, other essential factors such as cost-effectiveness, eco-friendliness, energy consumption, and human well-being can easily be overlooked. For instance, hydrazine, as a strong and common reductant [205-207] for the synthesis of AgNPs [149,208-211] is a highly toxic, cancerous, and lethal chemical [205]. Although some works have incorporated green synthesis techniques $[111,112,135,164,212,213]$, they do not completely eliminate the need for chemicals, nor do they rule out high-energy consumption in physical synthesis methods, thereby increasing costs greatly $[137,214]$.

Due to the disadvantages of physical and chemical methods, the focus has been directed towards the use of facile methods and materials that are less detrimental and more cost-effective. A common subject of interest in this area has been the selection of naturally occurring processes, widely known as "green processes". However, green synthesis of silver nanostructures is a general term defined as the production of silver nanostructures by using environmentally friendly techniques. Therefore, this method is not limited to synthesis by biological agents. For 
instance, alternative reagents such as ascorbic acid and sodium citrate are considered to synthesize AgNPs in a green and environmentally friendly manner [215]. The green synthesis of silver nanostructures has been receiving significant attention and is expected to rule out the use of hazardous chemical substances to a great extent [45,216-218], and also to disregard the use of high-energy consuming devices for the top-down synthesis of silver nanostructures [104,185]. Green synthesis of silver nanostructures has demonstrated great advances in the last decade, yet there are still issues regarding stability, size distribution, morphology, unknown biological functions [140], and the consideration that some biological processes cannot become industrially feasible due to strict and time-consuming aseptic procedures [185].

In this review, we first aim to discuss the most common AgNP synthesis methodologies and to compare them based on their cost, eco-friendliness, and energy consumption to show how green chemistry can improve the process and act as an alternative compared to physical and chemical synthesis. The physical and chemical synthesis methods are discussed only in terms of the process structure, advantages, and disadvantages. Therefore, a detailed study of physical and chemical methods for the synthesis of AgNPs is out of the scope for this review. Among the green synthesis procedures, significant attention has been recently given to plant-mediated synthesis because of its simple culturing procedures and potential for scale-up [181,219]. Various green synthesis studies from the literature were gathered and compared, giving one a clear and broad overview of the green synthesis processes. We then show how plant-mediated synthesis can emerge as a novel and alternative methodology towards the synthesis of 1D silver nanostructures, which have several applications in electronics. Finally, novel future directions including application of in situ characterization techniques in the course of reaction, continuous green and sustainable synthesis of silver nanostructures adaptable for in situ characterization, and incorporation of artificial intelligence (AI) in green silver nanostructures synthesis are discussed.

\section{Physical and chemical synthesis methodologies of silver nanoparticles}

In this section, chemical and physical synthesis methodologies of silver nanostructures are reviewed and their advantages and disadvantages are discussed. This section is reviewed to provide a comparison between chemical/physical synthesis and green synthesis of silver nanostructures. Since the main focus of this article is on reviewing the green synthesis of silver nanostructures as an alternative over chemical and physical methods, only the most common physical and chemical methods have been reviewed and the major part of our review has been dedicated to green synthesis of silver nanostructures.

\subsection{Physical synthesis}

Physical synthesis of silver nanoparticles includes methods that produce particles with dimensions of 1-100 nm from bulk silver, typically in the solid phase. It is also referred to as the top-down synthesis of silver nanoparticles. Unlike chemical and biological methods, physical techniques do not necessarily require using a reducing agent or stabilizer; however, they may be incorporated with other techniques.

2.1.1 Ball milling process. As one of the conventional processes, the ball milling process (mostly used as mechanochemical ball milling) is a method that is used commonly to produce AgNPs in a solid state [220]. Previously, AgNPs were produced using high-energy planetary ball milling [105,106,221]. Khayati et al. [105] utilized planetary ball milling in a mechanochemical process by adding organic process control agents (PCAs). In this work, depending on the type of PCA used, particle sizes varied from 14 to $34 \mathrm{~nm}$, and were of crystallite shape. In another work, AgNP crystallites were produced with an average size of $10-12 \mathrm{~nm}$ using the mechanochemical ball milling process by utilizing polyethylene glycol as the stabilizing agent [107]. The relatively small size of the produced AgNPs allowed effective antibacterial activity against Gram-positive and Gram-negative bacteria. The ball milling method is an inexpensive approach for the synthesis of AgNPs in a solid state [140], and can be used for the synthesis of AgNPs in ambient temperature, with a fair control over particle size [107]. It is a useful technique and synthesized nanoparticles may be used for antimicrobial applications [107]; however, there are several downsides with this method. The most common approach, provided by the literature, for the synthesis of NPs - especially AgNPs - is by laboratory-based planetary ball milling, which is insignificant for large-scale production [222]. In addition, the milling process itself may result in the creation of agglomerated products, especially during long processes, due to the large specific surface area of the produced NPs [222,223]. Furthermore, this technique is associated with substantial energy consumption - considering the milling period - compared to alternative methods [222].

2.1.2 Evaporation-condensation process. Evaporation-condensation is another conventional method used for the synthesis of AgNPs. This technique is applied by using a furnace processing chamber where the metal of interest is vaporized into a low-density gas phase, becomes supersaturated by decreasing temperature, and then is condensed to form nuclei which then grow into nanoparticles $[113,137]$. The chamber gas usually contains an inert gas such as helium or argon [113,224]. AgNPs synthesized using this technique were of crystalline shape with sizes from 7 to $55 \mathrm{~nm}[113,224]$. One of the advantages of this method is that it can potentially produce NPs on a large-scale 
[137], and can be used for long-term experiments [104,225]; however, there are significant disadvantages to using this technique. The required equipment is rigorous, very costly, and occupies a significantly large space [104,137,214]. Furthermore, due to the high operation temperature, a significant preheating time is required, and the process needs considerable time to achieve thermal stability [214,225]. There are also safety issues due to the high processing temperature which will elevate the surrounding environment temperature [137,226] Additionally, the process consumes a significant amount of energy $[214,225]$ due to the very high operating temperatures; thus, making it uneconomical.

2.1.3 Arc discharge process. Another physical method widely used for the synthesis of AgNPs is the arc discharge method. In this method, two electrodes - a cathode and an anode - are connected in a high current DC circuit and submerged in a solvent - mostly deionized water - to run the process $[129,131]$ These electrodes can be either composed of an inert metal, such as titanium, or any metal of interest of which the nanoparticles will be produced, for instance, silver for the synthesis of AgNPs $[129,131]$. In the case of titanium electrodes, $\mathrm{AgNO}_{3}$ is used as the precursor, an electric discharge takes place between cathode and anode, and an electron exchange takes place in the plasma region where silver ions are reduced [129]. In the case of silver electrodes, silver will be melted and vaporized from the electrode ends, and as a result, nanoparticles are formed from the silver condensates [131]. Tien et al. [227] synthesized AgNPs from 5 to $45 \mathrm{~nm}$ using the same method. In more recent works by Tseng et al. [132,133], AgNPs were produced using a microelectrical discharge machining system and by adding polyvinyl alcohol (PVA) as the capping agent. They obtained AgNPs with a diameter of 50-100 nm when PVA was not used, and a diameter in the range of 25-75 nm when PVA was used [132] The arc discharge method is advantageous in terms of the simplicity of the apparatus and equipment, low impurity due to the mere use of water, and fewer production steps [129]. In addition, this process can reach high NP synthesis rates in a short time [129,131]. However, the size distribution is large $[129,132,133]$, and the NPs produced have a fairly large size distribution compared to NPs produced by chemical methods.

2.1.4 Laser ablation process. A promising physical synthesis method used widely in recent years is laser ablation. This method is typically used for the synthesis of stable silver colloids either in solutions or in open air without utilizing any additional reagents $[108,112]$. AgNPs synthesized with this method are maintained in high purity due to the absence of chemical stabilizers and ligands, which provide NPs with unique surface characteristics [228]. Therefore, for safe biological applications (i.e. in the medical and food industry), this ap- proach would be preferred as an alternative for methods that necessarily require the use of chemical stabilizers [108,112]. In addition, the small NP size, low agglomeration rate, and narrow size distribution can be achieved by this method [108]. The process uses a laser beam at high energy to ablate pure $\mathrm{Ag}$ from which the separated AgNPs, either in liquid or vapor form, are attained and confined in the surrounding ambient [109]. The formation of nanoparticles by laser ablation depends on the thermal and optical properties of the utilized metal and the surrounding ambient conditions [228,229]. Despite its significant advantages, laser ablation presents some disadvantages that limit their use. In general, this method does not have high productivity and the utilization of laser ablation at an industrial scale is difficult. To achieve desired concentrations, highenergy lasers should be used, which increase the costs significantly [112].

2.1.5 Spray pyrolysis process. Spray pyrolysis is another method used to produce AgNPs with an average size of $10 \mathrm{~nm}$ embedded into amorphous calcium phosphate particles for enhanced adhesive applications [124]. The spray pyrolysis process requires using an atomizer, a tube furnace, a reaction tube, a collection filter, and a vacuum pump [124]. This method is also often used for production of metal powders and demonstrates less agglomeration, higher purity, and higher crystallinity compared to those produced by chemical methods [230]. The method is simple and reproducible [230]; however, the process runs using high operating temperatures, and more specifically, the center of the reaction tube may not reach the setpoint temperature due to the short residence time inside the reactor and finite heat transfer from the wall [124].

In general, although physical methods can produce nanoparticles with high purity, most of them are very expensive and may lead to agglomeration of products [140]. Based on all the disadvantages explained here, the mere use of physical methods may not be adequate for most cases to produce AgNPs with desired size, morphology, size distribution, and characteristics. Moreover, most physical approaches should be accompanied with chemical or green methodologies to compensate for deficiencies.

\subsection{Chemical synthesis}

Chemical reduction, or conventional chemical synthesis, is the most common approach for the synthesis of AgNPs [214]. This is performed by the presence of a metal precursor such as $\mathrm{AgNO}_{3}$, a reducing agent such as hydrazine, sodium borohydride, ethylene glycol, or dimethylformamide (DMF) as well as the presence of a stabilizer such as polyvinylpyrrolidone (PVP) or polyvinylalcohol (PVA). The chemical synthesis of nanoparticles is one of the bottom-up techniques due to the fact that par- 
ticles are formed from collective atoms in a nucleus rather than from the bulk [104].

2.2.1 Sol-gel process. The sol-gel method is one of the most common techniques to synthesize AgNPs. The sol-gel process is considered to be a multifaceted approach for the synthesis of nanoparticles in various forms - especially complex compounds - such as metal-complex oxides, inorganic nanocomposites, and chalcogenides [144]. In the sol-gel method, a gellike mixture is first prepared by mixing the silver precursor solution with a metal complex compound (i.e. comprised of $\mathrm{Ca}$, $\mathrm{Ti}, \mathrm{Sr}$, etc.) $[143,144]$ in a solvent such as water or alcohol Then the product is heated in order for the nucleation and reaction to take place [143,231]. In most cases, AgNPs are synthesized in metal oxide thin films such as $\mathrm{TiO}_{2}, \mathrm{SiO}_{2}$, and $\mathrm{ZrO}_{2}$ where the average particle size is almost $10 \mathrm{~nm}$ when the heating temperature is $600{ }^{\circ} \mathrm{C}$ in the case of $\mathrm{SiO}_{2}$ thin films, and $500{ }^{\circ} \mathrm{C}$ in the case of $\mathrm{TiO}_{2}$ and $\mathrm{ZrO}_{2}$ [231]. Arun Kumar et al. [145] synthesized AgNPs using the hydrolytic sol-gel method at 400,600 , and $800{ }^{\circ} \mathrm{C}$ with particles with an average size of $20 \mathrm{~nm}$ and crystalline shape. The sol-gel technique can also be performed at lower temperatures as well. Jadalannagari et al. [143] synthesized silver-doped hydroxyapatite nanorods using the sol-gel technique at $100{ }^{\circ} \mathrm{C}$ and produced particles with an average diameter of $25 \mathrm{~nm}$ with hexagonal cross section. In the sol-gel process, besides temperature and gel composition, the solvent plays an important role in determining the size, morphology, and surface characteristics of the synthesized AgNPs [232]. Organic solvents are generally more advantageous, as they can act as an oxygen-supplying agent for the metal oxide and result in more uniform structures and a smaller size distribution [232]. One important advantage of the sol-gel technique is the large freedom for choosing different precursors with various combinations (i.e. hybrid compounds), allowing the process to be adjusted accordingly in order to yield the desired complex product with tuned physiochemical characteristics [146]. In addition, combined with the hydrothermal approach (synthesis in a hot aqueous environment under high pressure), it can synthesize AgNPs at a lower temperature compared to the sol-gel process alone [144]. Nevertheless, there are some disadvantages regarding the application and feasibility of sol-gel-produced nanoparticles and nanocomposites. For instance, in industrial applications of nanoparticle-doped glasses such as those in the automotive industry, there exists some difficulty in the production of thick films larger than $1 \mu \mathrm{m}$ because of possible film cracks and shrinkage [233]. In addition to that, the film quality itself depends highly on process and environmental conditions such as temperature and humidity [233]. The sol-gel process is associated with costly precursors, process longevity, and difficulties regarding reproducibility [234].
2.2.2 Reverse-micelle process. The reverse micelle is another approach for the synthesis of AgNPs. Reverse micelles are produced from surfactants such as sucrose fatty acids in a hydrophobic solvent such as alkanes [235]. There is a water phase inside the microemulsions which is also referred to as the water pool where the reactants are present [235]. The water pool is where the silver ions are reduced into silver atoms which then form AgNPs [152]. The reverse micelle method has served as a common approach for the synthesis of AgNPs throughout the past two decades [152]. Regular reducing agents used in this method include sodium borohydride $\left(\mathrm{NaBH}_{4}\right)$ [236], hydrazine $\left(\mathrm{N}_{2} \mathrm{H}_{4}\right)$ [237], glucose [238], and quercetin [152,239], to name a few. The size and size distribution of the AgNPs is controlled by the strength of the reducing agent [152]. It was previously observed that hydrazine hydrate $\left(\mathrm{N}_{2} \mathrm{H}_{4} \cdot \mathrm{H}_{2} \mathrm{O}\right)$ can yield smaller AgNPs with a higher degree of dispersion compared to stronger reductants such as sodium borohydride $\left(\mathrm{NaBH}_{4}\right)$ [240]. Singha et al. [152] synthesized AgNPs in sodium dioctyl sulfosuccinate (AOT) reverse micelles using ascorbic acid as the reductant and obtained particles with an average size of $6 \mathrm{~nm}$. Yang et al. [154] used sodium borohydride as the reductant and octadecylamine (ODA) as the solvent and produced AgNPs with an average size of $3.38 \mathrm{~nm}$. The size distribution of the synthesized nanoparticles depends on the type of solvent as well as the reducing agent. This enables a method that provides many choices depending on the type of surfactant and solvent, which can be optimized to yield the desired size and morphology [152]. In addition, preparation of AgNPs using this method does not need any specific equipment, intense temperature, or pressure conditions [235]. The scale-up of the system is also relatively simple [235]. However, the downside of this method is the low productivity of particles per volume as a result of low reactant concentration inside the reverse micelles [235]. AOTmicroemulsions have been the most common microemulsions for the preparation of micelles [152]. However, this method may result in the synthesis of AgNPs with weak surface plasmon characteristics due to a broad surface plasmon band [152].

2.2.3 Chemical vapor deposition process. Chemical vapor deposition (CVD) and atomic layer deposition (ALD) are among other chemical methods for nanoparticle synthesis. CVD is a method that allows production of nanoparticles on a substrate [241]. The process consists of three steps. First, the addition of a volatile precursor in the gas phase to the reactor chamber. Second, adsorption of the vapor on the substrate surface and establishment of medium compounds followed by formation of a layer. Third, nucleation and growth of the layer through heating [241]. The important factors that control the process and size of synthesized AgNPs include the precursor introduction method, reactor pressure, gas flow 
properties, deposition rate, deposition duration, and substrate surface temperature $[157,241]$. The type of precursor appears to be the most significant factor in the process [241]. Silver nitrate is the most widely used precursor for this purpose [241]. An important advantage of this technique is the establishment of a silver-metal-oxide (e.g. $\mathrm{SiO}_{2}$ or $\mathrm{TiO}_{2}$ ) nanocomposite coating using only one deposition step [241]. The CVD method also provides many opportunities for the synthesis of silver-coated materials with varying size distribution and morphology [241]. The disadvantages of this method are associated with the high process cost, complexity, and low scale-up capability [158].

2.2.4 Wet chemical synthesis. Currently, most synthesis methods still rely on wet chemical reduction using a chemical reducing agent. The conventional wet synthesis of AgNPs using strong reductants such as sodium borohydride, hydrazine, and dimethylformamide (DMF) is currently the most common approach in the literature compared to other techniques previously described [214]. Although wet chemical methods can successfully offer narrow size distribution and can synthesize particles with small size, there are some major disadvantages regarding these methods. Additionally, toxicity and hazards can result from using these chemical substances. For instance, hydrazine and its derivative compounds (e.g. hydrazine hydrate) serve as a strong reductant in the synthesis of AgNPs [211] due to its strong reducing characteristic [242], however; hydrazine is known to be toxic and carcinogenic and causes severe damage to vital human organs such as the lungs [242,243]. The Environmental Protection Agency (EPA) has ranked hydrazine as a potential carcinogen with threshold limits as small as $10 \mathrm{ppb}$ [242,244]. $\mathrm{N}, \mathrm{N}$-Dimethylformamide (DMF) is also known as a strong and common reducing agent. However, the compound is reported to cause damage to the liver and digestive system [245]. Sodium borohydride, another strong reducing agent, is considered to have adverse effects on lungs and may cause pulmonary edema, which is a condition that results in fluid build-up in the lungs [246]. Besides the exposure risk during the process, an intense separation step should be considered to remove those compounds from the synthesized nanoparticles, thus making the process difficult and costly. In the case of hydrazine, the synthesized particles may potentially include some of the remnants from the reagent, thus making them hazardous or even unusable for biomedical applications. The polyol process is another common wet chemical method used for the synthesis of silver nanostructures. The polyol process is typically performed at $120-160{ }^{\circ} \mathrm{C}$ by utilizing ethylene glycol as the solvent and reducing agent and PVP as the capping agent in the presence of a small amount of salt mediator [247-249]. Although the polyol process is a non-hazardous and widely accepted method for the synthesis of silver nanostructures, there are some disadvantages associated with it. First, the polyol process is typically performed at temperatures higher than $120{ }^{\circ} \mathrm{C}$, which is associated with high energy consumption. Second, this process requires dilute concentrations of silver precursors ( $0.1 \mathrm{M}$ or lower) in order to maintain a uniform morphology and size distribution [250], which limits their scale-up capability. Third, the yield of the silver nanostructures is highly sensitive to reaction conditions, such as temperature, concentration ratio of PVP to $\mathrm{AgNO}_{3}$, salt mediator concentration, and the stirring type and rate [90]. On the other hand, natural compounds can be utilized to synthesize silver nanostructures in a much simpler fashion by enabling synthesis at room temperature and using only one reagent as both reducing and capping agent in addition to providing a non-hazardous and environmentally friendly route towards the synthesis of different silver nanostructures.

\section{Green synthesis of silver nanoparticles}

The green synthesis of nanoparticles can be defined as a set of techniques which utilize non-chemical reagents or nonhazardous methods for the production of nanoparticles. The main purpose of such methodologies is to minimize environmental toxicity and health-related hazards [104,214,251-253]. In most green silver nanostructure synthesis processes, the reduction of $\mathrm{Ag}^{+}$to $\mathrm{Ag}^{0}$ is performed either by biological species or by bio-based compounds derived from a desired type of plant or organism [252], which is also called biological synthesis. Bio-based reducing agents include microorganisms or biologically produced material. Microorganisms consisting of viruses, microalgae, fungi, yeast, and bacteria were previously used to synthesize AgNPs. Unlike physical synthesis, but similar to chemical synthesis, the biological synthesis of nanoparticles is classified under bottom-up approaches [104]. However, green synthesis is not limited to synthesis by bio-based compounds or biological species. For example, laser irradiation can also be used without using any reducing agent in order to synthesize AgNPs [252]. In addition, AgNPs were synthesized by microwave irradiation [254,255], ionizing irradiation [252,256], and pulse radiolysis [252]. Since these techniques may be utilized to produce nanoparticles with harmless procedures, they may also be classified under green synthesis methodologies. However, they have drawbacks in terms of energy consumption. A general comparison between chemical, physical, and green synthesis methods has been outlined in Table 1 . Figure 1 provides a clear overview of the classification of synthesis methods.

Nature has proven its unique ability to provide advice for synthesizing nanomaterials. The biogenic/green metal nanostructures' future is bright. Green processes are highly advantageous since they utilize naturally occurring processes to synthe- 
Table 1: Classification and comparison of various synthesis methodologies of silver nanostructures based on their advantages and disadvantages as reported in the literature.

\begin{tabular}{|c|c|c|c|}
\hline Method & Advantages & Disadvantages & Ref. \\
\hline \multicolumn{4}{|c|}{ Physical methods } \\
\hline ball milling & $\begin{array}{l}\text { - cheap } \\
\text { - process can be done at ambient } \\
\text { temperature }\end{array}$ & $\begin{array}{l}\text { - insignificant for large-scale } \\
\text { synthesis } \\
\text { - agglomeration of particles } \\
\text { - high energy consumption }\end{array}$ & {$[140,222,223,257,258]$} \\
\hline evaporation-condensation & $\begin{array}{l}\text { - large-scale synthesis } \\
\text { - useful for long-term experiments }\end{array}$ & $\begin{array}{l}\text { - rigorous equipment } \\
\text { - expensive equipment } \\
\text { - expensive process } \\
\text { - long preheating times } \\
\text { - high operating } \\
\text { temperatures } \\
\text { - high energy consumption }\end{array}$ & {$[104,137,214,225]$} \\
\hline arc discharge & $\begin{array}{l}\text { - simple equipment } \\
\text { - high purity of nanoparticles } \\
\text { - few process steps } \\
\text { - high synthesis rates }\end{array}$ & $\begin{array}{l}\text { - large size distribution of } \\
\text { nanoparticles }\end{array}$ & {$[129,131-133]$} \\
\hline laser ablation & $\begin{array}{l}\text { - high purity of nanoparticles } \\
\text { - small size of nanoparticles } \\
\text { - low agglomeration rate } \\
\text { - narrow size distribution }\end{array}$ & $\begin{array}{l}\text { - high energy consumption } \\
\text { - low productivity } \\
\text { - insignificant for large-scale } \\
\text { synthesis }\end{array}$ & {$[112,228,229]$} \\
\hline spray pyrolysis & $\begin{array}{l}\text { - high purity of nanoparticles } \\
\text { - process simplicity }\end{array}$ & $\begin{array}{l}\text { - high operating } \\
\text { temperatures } \\
\text { - low heat transfer to the } \\
\text { reaction tube due to low } \\
\text { residence time }\end{array}$ & {$[124,140,230]$} \\
\hline
\end{tabular}

Chemical methods

\begin{tabular}{|c|c|c|c|}
\hline sol-gel process & $\begin{array}{l}\text { - high purity and homogeneity of } \\
\text { nanoparticles } \\
\text { - can be efficiently used for } \\
\text { preparation of composites and } \\
\text { complex materials }\end{array}$ & $\begin{array}{l}\text { - limited industrial } \\
\text { applicability } \\
\text { - costly precursors } \\
\text { - process longevity } \\
\text { - difficulties regarding the } \\
\text { synthesis of monoliths } \\
\text { - reproducibility difficulties }\end{array}$ & {$[233,234]$} \\
\hline reverse micelle & $\begin{array}{l}\text { - process simplicity } \\
\text { - scale-up simplicity }\end{array}$ & $\begin{array}{l}\text { - low productivity of particles } \\
\text { per system volume } \\
\text { - large size distribution of } \\
\text { nanoparticles } \\
\text { - use of toxic and hazardous } \\
\text { chemicals such as hydrazine } \\
\text { and sodium borohydride }\end{array}$ & {$[152,235]$} \\
\hline chemical vapor deposition & $\begin{array}{l}\text { - preparation of nanocomposites } \\
\text { by only one deposition step }\end{array}$ & $\begin{array}{l}\text { - high process cost } \\
\text { - process complexity } \\
\text { - weak scale-up capability }\end{array}$ & {$[158,241]$} \\
\hline wet chemical synthesis & $\begin{array}{l}\text { - process simplicity } \\
\text { - narrow size distribution of } \\
\text { nanoparticles } \\
\text { - small size of nanoparticles }\end{array}$ & $\begin{array}{l}\text { - use of highly toxic and } \\
\text { hazardous substances such } \\
\text { as hydrazine, sodium } \\
\text { borohydride, and } \\
N, N \text {-dimethylformamide } \\
\text { (DMF) } \\
\text { - limited biomedical } \\
\text { applications }\end{array}$ & [242-245] \\
\hline
\end{tabular}


Table 1: Classification and comparison of various synthesis methodologies of silver nanostructures based on their advantages and disadvantages as reported in the literature. (continued)

Green/biological methods

\begin{tabular}{|c|c|c|c|}
\hline bacteriogenic synthesis & $\begin{array}{l}\text { - process simplicity } \\
\text { - environmentally-friendliness }\end{array}$ & $\begin{array}{l}\text { - pathogenic behavior of } \\
\text { certain species such as } \\
\text { E. coli } \\
\text { - significantly slow synthesis } \\
\text { rate } \\
\text { - large size distribution } \\
\text { - unknown biological } \\
\text { functions affecting the } \\
\text { synthesis process }\end{array}$ & {$[104,259]$} \\
\hline fungi-mediated synthesis & $\begin{array}{l}\text { - process simplicity } \\
\text { - environmentally-friendliness } \\
\text { - faster synthesis rate compared } \\
\text { to bacteriogenic synthesis } \\
\text { - high bioaccumulation capacity } \\
\text { and intracellular uptake } \\
\text { - less non-pathogenic behavior } \\
\text { compared to bacteriogenic } \\
\text { synthesis }\end{array}$ & $\begin{array}{l}\text { - process longevity } \\
\text { - pathogenic behavior } \\
\text { - unknown biological } \\
\text { functions affecting the } \\
\text { synthesis process }\end{array}$ & {$[104,178,179,260]$} \\
\hline virus/VLP-mediated synthesis & $\begin{array}{l}\text { - process simplicity } \\
\text { - environmentally-friendliness } \\
\text { - possibility to synthesize 1D } \\
\text { nanostructures } \\
\text { - small size of nanoparticles }\end{array}$ & $\begin{array}{l}\text { - lack of strong metal-binding } \\
\text { sites along the biotemplate } \\
\text { surface } \\
\text { - preparation of the } \\
\text { biotemplate is } \\
\text { time-consuming } \\
\text { - multiple coating cycles may } \\
\text { be required to yield a } \\
\text { uniform coating }\end{array}$ & {$[261-263]$} \\
\hline algae-mediated synthesis & $\begin{array}{l}\text { - process simplicity } \\
\text { - low cost } \\
\text { - environmentally-friendliness } \\
\text { - low reaction temperatures } \\
\text { - use of non-pathogenic and } \\
\text { non-hazardous reagents } \\
\text { - small size of nanoparticles } \\
\text { - uniform morphology of } \\
\text { nanoparticles }\end{array}$ & $\begin{array}{l}\text { - significantly Slow synthesis } \\
\text { rate } \\
\text { - unknown biological } \\
\text { functions affecting the } \\
\text { synthesis process }\end{array}$ & {$[264,265]$} \\
\hline plant/plant extract-mediated synthesis & $\begin{array}{l}\text { - process simplicity } \\
\text { - low cost } \\
\text { - environmentally-friendliness } \\
\text { - low reaction temperatures } \\
\text { - use of non-pathogenic and } \\
\text { non-hazardous reagents } \\
\text { - they can act as both reducing } \\
\text { and capping agent at the same } \\
\text { time } \\
\text { - broad scope }\end{array}$ & $\begin{array}{l}\text { - unknown mechanisms } \\
\text { affecting the synthesis } \\
\text { process }\end{array}$ & {$[104,140,187,188]$} \\
\hline
\end{tabular}

size nanoparticles. The idea was first introduced in the 19th century when scientists discovered the reducing ability of biological materials [130]. Green/biological methods provide several advantages over physical and chemical methods. They are eco-friendly unlike chemical methods [45,104], require less energy unlike physical methods [104,214], can be used for mass production [181], and are more economically feasible [104,181,219]. Sustainability, energy efficiency, renewability, and reduction of chemical derivatives, are among other advan- tages of green synthesis processes [266]. Furthermore, nature provides a high variety of reagents that can act as reducing agents, while in chemical methods the reagent selection including reducing, stabilizing, and capping agents are more limited. Therefore, recent works in the literature introduce highly diverse biological and green methods for the synthesis of AgNPs. However, it is critical to optimize these techniques not only in terms of scale-up capability, but also with respect to product quality and performance. Various green synthesis 


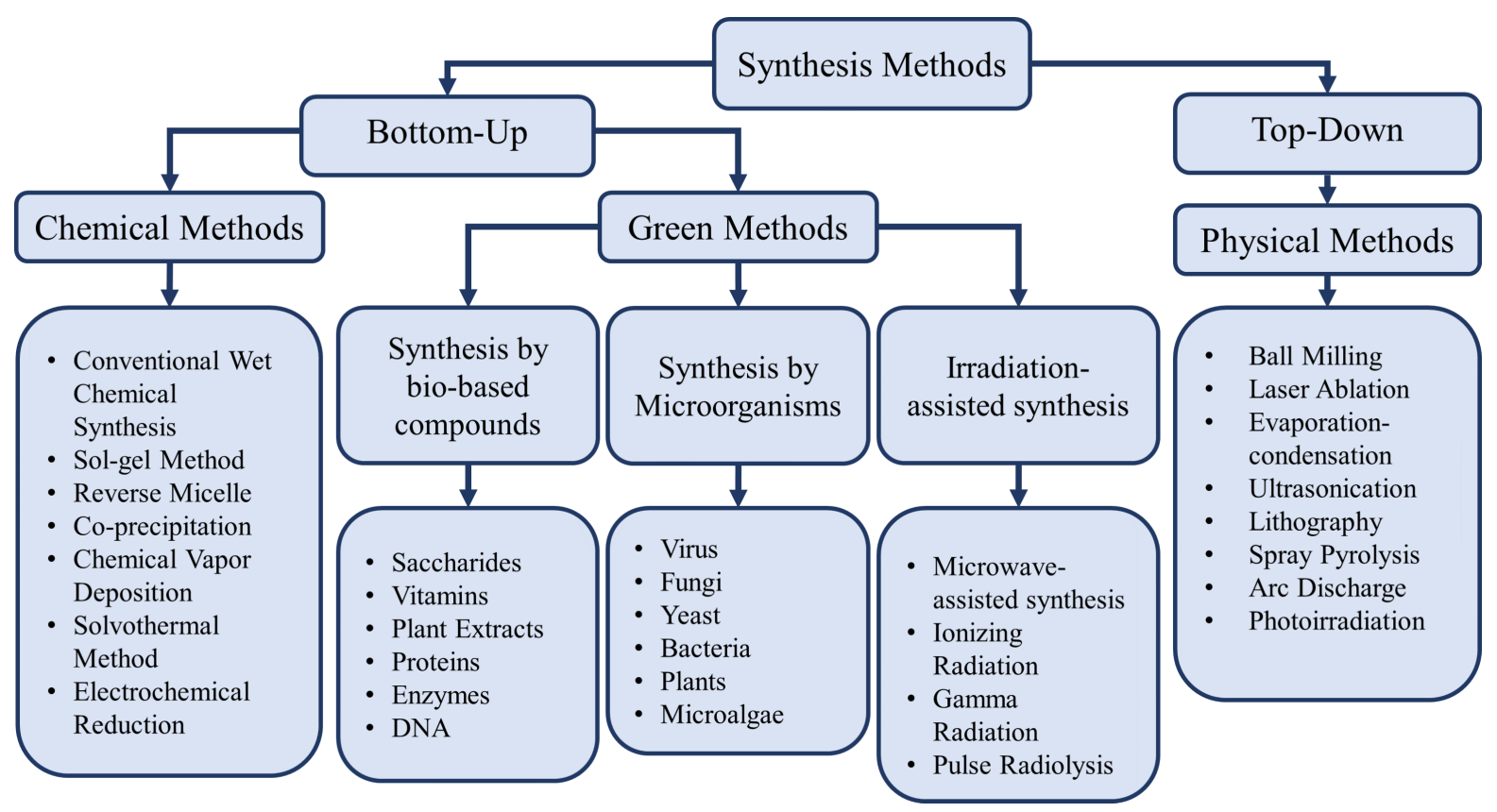

Figure 1: Classification of physical, chemical, and green synthesis methodologies of silver nanostructures with respect to reducing agent type, instruments, and processes.

studies of Ag nanostructures are reported in Table 2 along with the reaction conditions, size and morphology of synthesized Ag nanostructures, responsible functional groups in reduction, nucleation and growth, and applications of the synthesized $\mathrm{Ag}$ nanostructures.

\subsection{Bacteriogenic synthesis}

Bacteriogenic synthesis is a fairly advantageous process towards facile synthesis of AgNPs due to the reactive response of bacteria to silver. The Ag-resistant bacterial strains are utilized for the synthesis of AgNPs, as they can accumulate Ag atoms on the cell walls [104]. The bacteriogenic synthesis process can be either extracellular or intracellular. AgNPs are synthesized either by the biomass [267] or the cell culture supernatant [259]. AgNPs synthesized using bacteriogenic pathogens are commonly spherical in morphology and range from 5-200 $\mathrm{nm}$ in size. The mechanism through which AgNPs are synthesized is still not well understood; however, Fouriertransform infrared (FTIR) spectroscopy results from previous studies suggest that carboxylic and hydroxylic groups, in addition to primary and secondary amides corresponding to cellular proteins and enzymes, are responsible for the synthesis and stabilization of AgNPs (Table 2). These components are present in both the biomass and the cell culture supernatant. It was previously demonstrated that the synthesis rate can be faster for some bacterial strains including Escherichia coli (E. coli) and
K. Pneumonia when the cell culture supernatant is used [268]. However, the main downside of bacteriogenic synthesis is the slow synthesis rate and large size distribution compared to other green methods $[104,259]$. The applications of bacteria-synthesized AgNPs can be seen in the development of antimicrobial agents, biosensors, optics, solar energy, and drug delivery [267269].

\subsection{Fungi-mediated synthesis}

Fungal species have demonstrated significant potential for the synthesis of AgNPs. Their high binding and bioaccumulation capacity, intracellular uptake, and ease of handling provide them with additional advantages compared to bacteria [260]. Previous studies have shown that fungi-synthesis processes are followed by an enzymatic process, affecting the formation of stable AgNPs in the range of 5-15 nm [270]. However, this range can vary with respect to reaction conditions. FTIR results show that similar to bacteria, carbonyl, amide, and hydroxy groups corresponding to the cellular protein are responsible for the synthesis and stabilization of AgNPs [178,179]. The less non-pathogenic behavior of fungi and their faster synthesis rate suggest their use over bacteria. Fungi-synthesized AgNPs have proved to have noticeable anti-bacterial activity. Naqvi et al. showed that synthesized AgNPs using A. flavus fungi increased the biocidal effectiveness against drug-resistant bacteria significantly [271]. Fungi-assisted synthesis has proved to be a prom- 
ising approach towards the production of AgNPs; however, the pathogenic behavior of the fungi and long synthesis periods compared to other green methods makes it inferior among green synthesis processes.

\subsection{Plant virus template-mediated synthesis}

Plant-based viruses as biotemplates have been rarely used for the synthesis of silver nanostructures compared to other methods. To the best of our knowledge, there have been only a few studies [176,261-263] that have used plant viruses to synthesize silver nanostructures. In particular, tobacco mosaic virus (TMV) is used as the most common template to produce rod-shaped Ag nanostructures. However, TMV is not the only template that can be used for the synthesis of Ag nanostructures. Among others, Brome Mosaic Virus (BMV), Cowpea
Chlorotic Mottle Virus (CCMV), Cowpea Mosaic Virus (CPMV), Hibiscus Chlorotic Ringspot Virus (HCRSV), Red Clover Necrotic Mosaic Virus (RCNMV), and Turnip Yellow Mosaic Virus (TYMV) may also be used for the synthesis of Ag nanostructures [272]. The AgNPs can be synthesized either inside the viral template, within the interface, or on the outer surface [272]. In one of the early works, Dujardin et al. [261] synthesized AgNPs in cylindrical matrices using TMV as the biotemplate. In this work, AgNPs were $2-4 \mathrm{~nm}$ in size and it was shown that AgNPs, unlike platinum and gold nanoparticles, were coated on the inner surface of the TMV channel. The synthesis process is known to be mediated by the amino acid functional groups [263]. Although viral templates have not been investigated as much as other approaches, viral template-mediated $\mathrm{Ag}$ nanostructures have been demonstrating promising

Table 2: A review of various green processes, reaction conditions, necessary functional groups, and characterization techniques used for the synthesis of silver nanostructures with different sizes and morphologies along with their application.

\begin{tabular}{|c|c|c|c|c|}
\hline \multirow[t]{2}{*}{ Reducing agent } & Solvent & $\begin{array}{l}\text { Ingredient or functional group } \\
\text { responsible for } \mathrm{Ag}^{+} \text {reduction/particle } \\
\text { stabilization }\end{array}$ & Stabilizer & \multirow[t]{2}{*}{$\begin{array}{l}\text { Reaction time/ } \\
\text { temperature }\end{array}$} \\
\hline & Morphology/ dimensions & $\begin{array}{l}\text { Implemented characterization } \\
\text { techniques }\end{array}$ & $\begin{array}{l}\text { Application of the } \\
\text { synthesized } \mathrm{Ag} \\
\text { nanostructures }\end{array}$ & \\
\hline \multicolumn{5}{|c|}{ Bacteria/ bacteriogenic metabolites } \\
\hline \multirow{2}{*}{$\begin{array}{l}\text { exo-polysaccharide } \\
\text { (EPS) extract of } \\
\text { Bacillus safensis } \\
\text { LAU } 13\end{array}$} & not explicitly mentioned & $\begin{array}{l}\text { secondary amine and amide } \\
\text { (responsible for stabilization) }\end{array}$ & $\mathrm{N} / \mathrm{A}$ & $2 \mathrm{~h} / 30 \pm 2{ }^{\circ} \mathrm{C}$ \\
\hline & spherical/5-30 nm & FTIR/TEM/XRD & $\begin{array}{l}\text { antimicrobial } \\
\text { agents }\end{array}$ & {$[269]$} \\
\hline \multirow[t]{2}{*}{ Bacillus krulwichiae } & distilled water & $\begin{array}{l}\text { carboxyl, hydroxy, aldehyde, ester } \\
\text { groups responsible for reduction and } \\
\text { stabilization }\end{array}$ & $N / A$ & $\begin{array}{l}24 \mathrm{~h} / \text { room } \\
\text { temperature }\end{array}$ \\
\hline & $\begin{array}{l}\text { spherical/av.: } \\
25.88 \pm 10.49 \mathrm{~nm}\end{array}$ & FTIR/SEM/XRD/EDX & $\begin{array}{l}\text { antimicrobial/anti- } \\
\text { biofilm agents }\end{array}$ & {$[280]$} \\
\hline \multirow[t]{2}{*}{$\begin{array}{l}\text { Bacillus } \\
\text { cellulosilyticus (EPS } \\
\text { extract) }\end{array}$} & distilled water & $\begin{array}{l}\text { carboxyl, hydroxy, aldehyde/ester } \\
\text { groups responsible for reduction and } \\
\text { stabilization }\end{array}$ & $\mathrm{N} / \mathrm{A}$ & $\begin{array}{l}24 \mathrm{~h} / \text { room } \\
\text { temperature }\end{array}$ \\
\hline & $\begin{array}{l}\text { spherical/av.: } \\
23.99 \pm 8.43 \mathrm{~nm}\end{array}$ & FTIR/SEM/XRD/EDX & $\begin{array}{l}\text { antimicrobial/anti- } \\
\text { biofilm agents }\end{array}$ & [280] \\
\hline \multirow[t]{2}{*}{ Bacillus brevis } & not explicitly mentioned & $\begin{array}{l}\text { amide }(-\mathrm{CONH}) \text {, hydroxy, and } \\
\text { carbonyl corresponding to the protein } \\
\text { (responsible for reduction) } / \mathrm{C}=\mathrm{C} \text { and } \\
-\mathrm{CH} \text { on protein responsible for } \\
\text { stabilization }\end{array}$ & $\mathrm{N} / \mathrm{A}$ & $\begin{array}{l}24 \mathrm{~h} / \text { room } \\
\text { temperature }\end{array}$ \\
\hline & spherical/41-68 nm & AFM/SEM/TLC/FTIR & $\begin{array}{l}\text { antimicrobial } \\
\text { agents }\end{array}$ & {$[168]$} \\
\hline \multirow[t]{2}{*}{ Enterobacter cloacae } & not explicitly mentioned & primary amide, secondary amide & $\mathrm{N} / \mathrm{A}$ & $\begin{array}{l}72 \mathrm{~h} / \text { room } \\
\text { temperature }\end{array}$ \\
\hline & spherical/12-30 nm & FTIR/SEM/XRD & $\begin{array}{l}\text { antibacterial } \\
\text { activity against } \\
\text { MDR strains }\end{array}$ & [281] \\
\hline
\end{tabular}


Table 2: A review of various green processes, reaction conditions, necessary functional groups, and characterization techniques used for the synthesis of silver nanostructures with different sizes and morphologies along with their application. (continued)

\begin{tabular}{|c|c|c|c|c|}
\hline \multirow[t]{2}{*}{$\begin{array}{l}\text { EPS from } \\
\text { Leuconostoc lactis }\end{array}$} & Milli-Q water & hydroxy groups & $\mathrm{N} / \mathrm{A}$ & $\begin{array}{l}1 \text { month/room } \\
\text { temperature }\end{array}$ \\
\hline & spherical/30-200 nm & $\begin{array}{l}\text { SEM/TEM/XRD/UV-vis/AFM/TGA-D } \\
\text { TA }\end{array}$ & $\begin{array}{l}\text { textile industry: } \\
\text { degradation of } \\
\text { azo-dyes }\end{array}$ & {$[169]$} \\
\hline \multirow[t]{2}{*}{$\begin{array}{l}\text { Pseudomonas } \\
\text { stutzeri }\end{array}$} & not explicitly mentioned & $\begin{array}{l}\text { primary, secondary, and aliphatic } \\
\text { amides }\end{array}$ & $N / A$ & $8 \mathrm{~h} / 80^{\circ} \mathrm{C}$ \\
\hline & spherical/15-20 nm & TEM/FTIR & $\begin{array}{l}\text { antibacterial } \\
\text { activity against } \\
\text { MDR strains }\end{array}$ & {$[170]$} \\
\hline $\begin{array}{l}\text { Bacillus } \\
\text { methylotrophicus }\end{array}$ & $\begin{array}{l}\text { not explicitly mentioned } \\
\text { spherical/10-30 nm }\end{array}$ & $\begin{array}{l}\text { extracellular enzymes and proteins } \\
\text { FE-TEM/EDX/UV-vis }\end{array}$ & $\begin{array}{l}\mathrm{N} / \mathrm{A} \\
\text { antibacterial } \\
\text { activity }\end{array}$ & $\begin{array}{l}-/ 28^{\circ} \mathrm{C} \\
{[282]}\end{array}$ \\
\hline \multirow[t]{2}{*}{$\begin{array}{l}\text { Escherichia coli } \\
\text { (culture supernatant) }\end{array}$} & not explicitly mentioned & $\begin{array}{l}\text { primary and secondary amines } \\
\text { corresponding to the protein }\end{array}$ & $\mathrm{N} / \mathrm{A}$ & $\begin{array}{l}24 \mathrm{~h} / \text { room } \\
\text { temperature }\end{array}$ \\
\hline & $\begin{array}{l}\text { spherical/40-90 nm } \\
\text { (unoptimized condition) } \\
\text { and } 10-40 \mathrm{~nm} \\
\text { (optimized condition) }\end{array}$ & XRD/TEM & $\begin{array}{l}\text { conductivity/ } \\
\text { catalytic and } \\
\text { antimicrobial } \\
\text { activity }\end{array}$ & [283] \\
\hline \multirow{2}{*}{$\begin{array}{l}\text { Staphylococcus } \\
\text { aureus }\end{array}$} & Milli-Q water & extracellular enzymes & $\mathrm{N} / \mathrm{A}$ & $5 \mathrm{~min} /-$ \\
\hline & $\begin{array}{l}\text { varied } \\
\text { morphology/160-180 } \\
\mathrm{nm}\end{array}$ & AFM/UV-vis & $\begin{array}{l}\text { antimicrobial } \\
\text { activity }\end{array}$ & {$[284]$} \\
\hline \multirow[t]{2}{*}{ cyanobacteria } & Milli-Q water & - & $\mathrm{N} / \mathrm{A}$ & $60 \mathrm{~min} / 30-60^{\circ} \mathrm{C}$ \\
\hline & spherical/60-80 nm & UV-vis/DLS/TEM & $\begin{array}{l}\text { dye } \\
\text { decolorization } \\
\text { property }\end{array}$ & [285] \\
\hline \multicolumn{5}{|c|}{ Yeast } \\
\hline $\begin{array}{l}\text { Cryptococcus } \\
\text { laurentii (culture } \\
\text { supernatant) }\end{array}$ & $\begin{array}{l}\text { not explicitly mentioned } \\
\text { spherical/av.: } \\
35 \text { and } 400 \mathrm{~nm}\end{array}$ & $\begin{array}{l}\text { amide corresponding to peptides } \\
\text { UV-vis/TEM/XRD/FTIR }\end{array}$ & $\begin{array}{l}\mathrm{N} / \mathrm{A} \\
\text { antifungal activity }\end{array}$ & $\begin{array}{l}48 \mathrm{~h} / 28 \pm 4{ }^{\circ} \mathrm{C} \\
{[178]}\end{array}$ \\
\hline \multirow[t]{2}{*}{$\begin{array}{l}\text { Saccharomyces } \\
\text { cerevisiae }\end{array}$} & not explicitly mentioned & $\begin{array}{l}\text { cytoplasmic enzymes } \\
\text { (oxidoreductase and oxidase) } \\
\text { responsible for reduction/proteins } \\
\text { responsible for stabilization }\end{array}$ & $\mathrm{N} / \mathrm{A}$ & $72 \mathrm{~h} / 25^{\circ} \mathrm{C}$ \\
\hline & spherical/2-20 nm & TEM/DLS & $\begin{array}{l}\text { cosmetics, foods, } \\
\text { and consumer } \\
\text { goods }\end{array}$ & {$[179]$} \\
\hline
\end{tabular}

Plant/fruit extracts

\begin{tabular}{|c|c|c|c|c|}
\hline \multirow[t]{2}{*}{$\begin{array}{l}\text { green tea powder } \\
\text { (Camelia Sinensis) }\end{array}$} & deionized water & $\begin{array}{l}\text { tea polyphenols (responsible for } \\
\text { stabilization) }\end{array}$ & $\mathrm{N} / \mathrm{A}$ & $15 \mathrm{~min} / 60^{\circ} \mathrm{C}$ \\
\hline & $\begin{array}{l}\text { spherical/ } \\
34.68 \pm 4.95 \mathrm{~nm}\end{array}$ & $\begin{array}{l}\text { UV-vis/XRD/FTIR/TGA/AFM/DLS/ } \\
\text { XPS }\end{array}$ & $\begin{array}{l}\text { antibacterial } \\
\text { activity }\end{array}$ & {$[286]$} \\
\hline \multirow[t]{2}{*}{$\begin{array}{l}\text { Camelia Sinensis } \\
\text { extract }\end{array}$} & deionized water & not mentioned & $\mathrm{N} / \mathrm{A}$ & $\begin{array}{l}30 \text { days/room } \\
\text { temperature }\end{array}$ \\
\hline & $\begin{array}{l}\text { one-dimensional } \\
\text { (nanowires)/ } \\
50 \mathrm{~nm} \text { (diameter) and } \\
1.3 \mu \mathrm{m} \text { (length) }\end{array}$ & FESEM/HRTEM/HAADF/UV-vis & $\begin{array}{l}\text { antibacterial } \\
\text { activity }\end{array}$ & {$[287]$} \\
\hline
\end{tabular}


Table 2: A review of various green processes, reaction conditions, necessary functional groups, and characterization techniques used for the synthesis of silver nanostructures with different sizes and morphologies along with their application. (continued)

\begin{tabular}{|c|c|c|c|c|}
\hline $\begin{array}{l}\text { blackberry fruit } \\
\text { extract }\end{array}$ & $\begin{array}{l}\text { deionized water } \\
\text { spherical/12-50 nm }\end{array}$ & $\begin{array}{l}\mathrm{O}-\mathrm{H} \text { and } \mathrm{C}=\mathrm{O} \text { groups } \\
\text { TEM/FTIR/DLS/XRD/UV-vis }\end{array}$ & $\begin{array}{l}\mathrm{N} / \mathrm{A} \\
\text { antioxidant } \\
\text { activity }\end{array}$ & $\begin{array}{l}48 \mathrm{~h} / 25^{\circ} \mathrm{C} \\
{[288]}\end{array}$ \\
\hline \multirow[t]{2}{*}{$\begin{array}{l}\text { Berberis Vulgaris leaf } \\
\text { extract }\end{array}$} & distilled water & not mentioned & $\mathrm{N} / \mathrm{A}$ & $\begin{array}{l}1 \mathrm{~h} / \text { room } \\
\text { temperature }\end{array}$ \\
\hline & spherical/30-70 nm & TEM/XRD/DLS/UV-vis & $\begin{array}{l}\text { antibacterial } \\
\text { activity }\end{array}$ & {$[187]$} \\
\hline \multirow{2}{*}{$\begin{array}{l}\text { Origanum vulgare } \\
\text { extract }\end{array}$} & deionized water & phytomolecules & $\mathrm{N} / \mathrm{A}$ & $2 \mathrm{~h} / 85-90^{\circ} \mathrm{C}$ \\
\hline & spherical/2-25 nm & UV-vis/XRD/HRTEM/EDX/FTIR & $\begin{array}{l}\text { antibacterial and } \\
\text { antifungal activity }\end{array}$ & {$[186]$} \\
\hline \multirow[t]{2}{*}{$\begin{array}{l}\text { Coffea Arabica seed } \\
\text { extract }\end{array}$} & deionized water & phenolic groups & $\mathrm{N} / \mathrm{A}$ & $\begin{array}{l}2 \mathrm{~h} / \text { room } \\
\text { temperature }\end{array}$ \\
\hline & $\begin{array}{l}\text { spherical and } \\
\text { ellipsoidal/20-30 nm }\end{array}$ & UV-vis/SEM/SEM-EDXA/FTIR/DLS & $\begin{array}{l}\text { antibacterial } \\
\text { activity }\end{array}$ & {$[289]$} \\
\hline \multirow[t]{2}{*}{ lignin } & Milli-Q water & phenolic hydroxy groups & $\mathrm{N} / \mathrm{A}$ & $30 \mathrm{~min} / 85^{\circ} \mathrm{C}$ \\
\hline & $\begin{array}{l}\text { spherical } / 7.3 \pm 2.2 \mathrm{~nm} \\
\text { and } 14.3 \pm 1.8 \mathrm{~nm}\end{array}$ & UV-vis/TEM & not mentioned & {$[290]$} \\
\hline \multirow[t]{2}{*}{ tannin } & not explicitly mentioned & hydroxy groups & $\mathrm{N} / \mathrm{A}$ & $2 \mathrm{~h} / 50^{\circ} \mathrm{C}$ \\
\hline & $\begin{array}{l}\text { one-dimensional } \\
\text { (nanowires)/ } \\
50 \mathrm{~nm} \text { (diameter) }\end{array}$ & UV-vis/HRTEM/SEM/SAED/IR & $\begin{array}{l}\text { detection of } \mathrm{Pb}(\mathrm{II}) \\
\text { ions }\end{array}$ & {$[291]$} \\
\hline \multirow[t]{2}{*}{ clove oil } & not explicitly mentioned & hydroxy groups from eugenol & $\mathrm{N} / \mathrm{A}$ & $\begin{array}{l}\text { not mentioned/room } \\
\text { temperature }\end{array}$ \\
\hline & $\begin{array}{l}\text { one-dimensional } \\
\text { (nanowires)/ } \\
39 \pm 0.01 \mathrm{~nm} \text { (diameter) } \\
\text { and } 3 \mu \mathrm{m} \text { (length) }\end{array}$ & UV-vis/HRTEM/XRD/FTIR & conductive ink & {$[292]$} \\
\hline \multirow[t]{2}{*}{$\begin{array}{l}\text { apple extract } \\
\text { (microwave-assisted) }\end{array}$} & ultrapure water & not mentioned & $\mathrm{N} / \mathrm{A}$ & $\begin{array}{l}2.5 \mathrm{~h} \text { for } \\
\text { microwave-assisted } \\
\text { reduction/room } \\
\text { temperature and } \\
96 \mathrm{~h} \text { in thermally } \\
\text { assisted one-pot } \\
\text { reduction } / 100^{\circ} \mathrm{C}\end{array}$ \\
\hline & $\begin{array}{l}\text { spherical/ } \\
28.24 \pm 1.15 \mathrm{~nm} \text { for } \\
\text { thermally assisted and } \\
22.05 \pm 1.05 \mathrm{~nm} \text { for } \\
\text { microwave-assisted } \\
\text { reaction }\end{array}$ & UV-vis/XRD/EDS & $\begin{array}{l}\text { antibacterial } \\
\text { activity }\end{array}$ & [293] \\
\hline \multirow[t]{2}{*}{ Azadirachta indica } & not explicitly mentioned & $\begin{array}{l}\mathrm{C}=\mathrm{O} \text { groups corresponding to alkyne } \\
\text { groups, } \mathrm{C}-\mathrm{O} \text { and } \mathrm{C}-\mathrm{O}-\mathrm{C} \text { bonds } \\
\text { corresponding to flavonoids and } \\
\text { terpenoids (responsible for } \\
\text { stabilization) }\end{array}$ & $\mathrm{N} / \mathrm{A}$ & $\begin{array}{l}15 \mathrm{~min} / \mathrm{room} \\
\text { temperature }\end{array}$ \\
\hline & spherical/34 nm & UV-vis/FTIR/TEM/DLS & $\begin{array}{l}\text { antibacterial } \\
\text { activity }\end{array}$ & {$[294]$} \\
\hline \multirow[t]{2}{*}{ Elephantopus Scaber } & distilled water & $\begin{array}{l}\mathrm{O}-\mathrm{H}, \mathrm{C}=\mathrm{O} \text { stretching of plant } \\
\text { constituents and } \mathrm{C}=\mathrm{C} \text { stretching of } \\
\text { aromatic rings }\end{array}$ & $\mathrm{N} / \mathrm{A}$ & $30 \mathrm{~min} / 40^{\circ} \mathrm{C}$ \\
\hline & spherical/37.86 nm & UV-vis/TEM/XRD/FTIR & $\begin{array}{l}\text { antimicrobial and } \\
\text { anticancer activity }\end{array}$ & {$[255]$} \\
\hline
\end{tabular}


Table 2: A review of various green processes, reaction conditions, necessary functional groups, and characterization techniques used for the synthesis of silver nanostructures with different sizes and morphologies along with their application. (continued)

\begin{tabular}{|c|c|c|c|c|}
\hline \multirow[t]{2}{*}{$\begin{array}{l}\text { Cinnamon } \\
\text { zeylanicum }\end{array}$} & $\begin{array}{l}\text { dimethyl sulfoxide } \\
\text { (DMSO)/ethanol/distilled } \\
\text { water }\end{array}$ & $\begin{array}{l}\text { aldehyde content (responsible for } \\
\text { reduction) }\end{array}$ & $\mathrm{N} / \mathrm{A}$ & $2 \mathrm{~h} / 60^{\circ} \mathrm{C}$ \\
\hline & $\begin{array}{l}\text { spherical/2-10 nm (in } \\
\text { ethanol), } \\
\text { polygonal/5-25 nm (in } \\
\text { distilled water), } \\
\text { spherical/10-50 nm (in } \\
\text { DMSO) }\end{array}$ & UV-vis/HRTEM & $\begin{array}{l}\text { antibacterial } \\
\text { activity }\end{array}$ & {$[295]$} \\
\hline \multirow[t]{2}{*}{ banana peel extract } & distilled water & $\begin{array}{l}\text { carboxyl, hydroxy and amide groups } \\
\text { (responsible for reduction) }\end{array}$ & $\mathrm{N} / \mathrm{A}$ & $76 \mathrm{~h} / 100{ }^{\circ} \mathrm{C}$ \\
\hline & spherical/23.7 nm & UV-vis/EDX/XRD/SEM/TEM/FTIR & $\begin{array}{l}\text { antibacterial } \\
\text { activity }\end{array}$ & {$[296]$} \\
\hline \multirow[t]{2}{*}{ turmeric extract } & Milli-Q water & $\begin{array}{l}\text { hydroxy groups present in the } \\
\text { curcumin powder and } \mathrm{C}-\mathrm{H} \text { bonds in } \\
\text { turmeric powder responsible for the } \\
\text { reduction }\end{array}$ & $\mathrm{N} / \mathrm{A}$ & $\begin{array}{l}24 \mathrm{~h} / \text { room } \\
\text { temperature }\end{array}$ \\
\hline & spherical/18 $\pm 0.5 \mathrm{~nm}$ & UV-vis/TEM/EDX/FTIR & $\begin{array}{l}\text { antibacterial } \\
\text { activity }\end{array}$ & {$[297]$} \\
\hline \multirow[t]{2}{*}{ marigold flower } & ultra-purified water & $\begin{array}{l}\mathrm{N}-\mathrm{H} \text { amid stretching, } \mathrm{C}-\mathrm{H} \text { stretching } \\
\text { from vinyl disubstituted alkenes, and } \\
\mathrm{C}-\mathrm{Cl} \text { stretching from alkyl halides } \\
\text { (responsible for reduction) }\end{array}$ & $\mathrm{N} / \mathrm{A}$ & $\begin{array}{l}24 \mathrm{~h} / \text { room } \\
\text { temperature }\end{array}$ \\
\hline & $\begin{array}{l}\text { varied (spherical, } \\
\text { hexagonal, and } \\
\text { irregular) } / 46.11 \mathrm{~nm}\end{array}$ & UV-vis/XRD/FTIR/EDX/SAED/TEM & $\begin{array}{l}\text { antibacterial } \\
\text { activity }\end{array}$ & {$[298]$} \\
\hline \multirow[t]{2}{*}{ starch } & Milli-Q water & $\begin{array}{l}\mathrm{O}-\mathrm{H} \text { stretching (aliphatic hydroxy } \\
\text { group) and } \mathrm{C}=\mathrm{O} \text { (responsible for } \\
\text { reduction and stabilization) }\end{array}$ & $\mathrm{N} / \mathrm{A}$ & $48 \mathrm{~h} / 25^{\circ} \mathrm{C}$ \\
\hline & $\begin{array}{l}\text { spherical, polydispersed } \\
\text { and amorphous } / 45.6 \mathrm{~nm}\end{array}$ & UV-vis/TEM/SAED/XRD/FTIR/DLS & catalytic activity & {$[299]$} \\
\hline \multirow[t]{2}{*}{ ginger } & deionized water & phenolic groups and flavonoids & $\mathrm{N} / \mathrm{A}$ & $\begin{array}{l}2 \mathrm{~h} / \text { room } \\
\text { temperature }\end{array}$ \\
\hline & $\begin{array}{l}\text { spherical/ } \\
10.10-18.33 \mathrm{~nm}\end{array}$ & UV-vis/TEM & $\begin{array}{l}\text { antioxidant and } \\
\text { antimicrobial } \\
\text { activity }\end{array}$ & [300] \\
\hline \multicolumn{5}{|c|}{ Vitamins } \\
\hline \multirow{2}{*}{$\begin{array}{l}\text { vitamin } \mathrm{C} \text { (ascorbic } \\
\text { acid) }\end{array}$} & high purity water & not mentioned & citrate & $15 \mathrm{~min} / 30^{\circ} \mathrm{C}$ \\
\hline & quasi-spherical/31 nm & UV-vis/TEM & $\begin{array}{l}\text { not specifically } \\
\text { mentioned }\end{array}$ & [301] \\
\hline \multirow[t]{2}{*}{$\begin{array}{l}\text { vitamin B2 } \\
\text { (riiboflavin) }\end{array}$} & Milli-Q water & not mentioned & $\mathrm{N} / \mathrm{A}$ & $\begin{array}{l}24 \mathrm{~h} / \text { room } \\
\text { temperature }\end{array}$ \\
\hline & $\begin{array}{l}\text { spherical/6.1 } \pm 0.1 \mathrm{~nm} \text {, } \\
\text { nanorods }(10-20 \mathrm{~nm} \\
\text { diameter, } 100-200 \mathrm{~nm} \\
\text { length) }\end{array}$ & UV-vis/TEM/SEM/EDX & $\begin{array}{l}\text { catalytic } \\
\text { polymerization of } \\
\text { aniline and } \\
\text { pyrrole }\end{array}$ & {$[204]$} \\
\hline $\begin{array}{l}\text { vitamin B12 } \\
\text { (microwave-assisted) }\end{array}$ & $\begin{array}{l}\text { Milli-Q water } \\
\text { irregular/70-600 nm }\end{array}$ & $\begin{array}{l}\text { not mentioned } \\
\text { UV-vis/XRD/TEM/SEM }\end{array}$ & $\begin{array}{l}\mathrm{N} / \mathrm{A} \\
\text { not specifically } \\
\text { mentioned }\end{array}$ & $\begin{array}{l}3-6 \mathrm{~min} / 100{ }^{\circ} \mathrm{C} \\
{[302]}\end{array}$ \\
\hline
\end{tabular}


Table 2: A review of various green processes, reaction conditions, necessary functional groups, and characterization techniques used for the synthesis of silver nanostructures with different sizes and morphologies along with their application. (continued)

\begin{tabular}{|c|c|c|c|c|}
\hline \multirow[t]{2}{*}{$\begin{array}{l}\text { Chlorococcum } \\
\text { humicola }\end{array}$} & distilled sterile water & $\begin{array}{l}\mathrm{C}-\mathrm{N} \text { bonds corresponding to } \\
\text { aromatic and aliphatic amines } \\
\text { (responsible for stabilization) }\end{array}$ & $\mathrm{N} / \mathrm{A}$ & $\begin{array}{l}48 \mathrm{~h} / \text { room } \\
\text { temperature }\end{array}$ \\
\hline & spherical/2-16 nm & SEM/TEM/FTIR/UV-vis/XRD & $\begin{array}{l}\text { antibacterial } \\
\text { activity }\end{array}$ & [278] \\
\hline \multirow[t]{2}{*}{ Spirulina } & $\begin{array}{l}\text { sterilized } \\
\text { double-distilled water }\end{array}$ & $\begin{array}{l}\mathrm{O}-\mathrm{H} \text { groups, } \mathrm{C}-\mathrm{O} \text { bonds } \\
\text { corresponding to } \mathrm{COOH} \text { group, and } \\
\mathrm{N}-\mathrm{H} \text { bonds corresponding to primary } \\
\text { and secondary amines }\end{array}$ & $\mathrm{N} / \mathrm{A}$ & $\begin{array}{l}3,6,9 \text { and } 12 \mathrm{~h} / \mathrm{room} \\
\text { temperature }\end{array}$ \\
\hline & spherical/5-50 nm & UV-vis/FTIR/XRD/EDX/bio-TEM & $\begin{array}{l}\text { antibacterial } \\
\text { activity }\end{array}$ & [279] \\
\hline \multirow[t]{2}{*}{ Chlorella Vulgaris } & not explicitly mentioned & $\begin{array}{l}\mathrm{C}=\mathrm{O} \text { and }-\mathrm{H} \text { (amide I and amide II) } \\
\text { bonds corresponding to proteins } \\
\text { (responsible for stabilization and } \\
\text { reduction) }\end{array}$ & $\mathrm{N} / \mathrm{A}$ & 5 days $/ 25^{\circ} \mathrm{C}$ \\
\hline & spherical $/ 9.8 \pm 5.7 \mathrm{~nm}$ & UV-vis/XRD/TEM/FTIR/EDX & $\begin{array}{l}\text { antibacterial } \\
\text { activity }\end{array}$ & {$[275]$} \\
\hline \multirow[t]{2}{*}{ Padina pavonia } & not explicitly mentioned & $\begin{array}{l}\mathrm{N}-\mathrm{H} \text { (amine), } \mathrm{C}-\mathrm{N} \text { (primary amines) } \\
\text { groups and } \mathrm{O}-\mathrm{H} \text { groups (responsible } \\
\text { for reduction) }\end{array}$ & $\mathrm{N} / \mathrm{A}$ & $\begin{array}{l}3 \mathrm{~h} / \text { room } \\
\text { temperature }\end{array}$ \\
\hline & $\begin{array}{l}\text { spherical, triangular, } \\
\text { rectangle, polyhedral } \\
\text { and hexagonal/ } \\
49.58-86.37 \mathrm{~nm}\end{array}$ & UV-vis/TEM/DLS/FTIR & $\begin{array}{l}\text { not specifically } \\
\text { mentioned }\end{array}$ & [193] \\
\hline \multirow[t]{2}{*}{$\begin{array}{l}\text { Chlamydomonas } \\
\text { reinhardtii }\end{array}$} & deionized water & amine and carbohydrate groups & $\mathrm{N} / \mathrm{A}$ & $\begin{array}{l}192 \mathrm{~h} / \text { room } \\
\text { temperature }\end{array}$ \\
\hline & spherical/5.6 $\pm 2.4 \mathrm{~nm}$ & UV-vis/XRD/TEM & $\begin{array}{l}\text { not specifically } \\
\text { mentioned }\end{array}$ & {$[192]$} \\
\hline \multicolumn{5}{|c|}{ Viruses } \\
\hline \multirow[t]{2}{*}{ tobacco mosaic virus } & not explicitly mentioned & $\begin{array}{l}\text { hydroxy, carboxyl and thiol groups } \\
\text { (responsible for reduction) }\end{array}$ & $\mathrm{N} / \mathrm{A}$ & $1 \mathrm{~h} / 50{ }^{\circ} \mathrm{C}$ \\
\hline & spherical/2 nm & UV-vis/TEM & $\begin{array}{l}\text { catalytic and } \\
\text { antibacterial } \\
\text { activities }\end{array}$ & {$[176]$} \\
\hline
\end{tabular}

\begin{tabular}{|c|c|c|c|c|}
\hline \multicolumn{5}{|c|}{ Fungus } \\
\hline Rhizopus stolonifer & deionized water & $\begin{array}{l}\text { carbonyl group of amino-acid residue } \\
\text { and peptide-protein }\end{array}$ & $\mathrm{N} / \mathrm{A}$ & 2 days $/ 40{ }^{\circ} \mathrm{C}$ \\
\hline & spherical/2.86 $\pm 0.3 \mathrm{~nm}$ & UV-vis/XRD/HR-TEM/FTIR & $\begin{array}{l}\text { not specifically } \\
\text { mentioned }\end{array}$ & {$[303]$} \\
\hline \multirow[t]{2}{*}{ Penicillium aculeatum } & Milli-Q deionized water & $\begin{array}{l}\mathrm{N}-\mathrm{H} \text { and } \mathrm{C}=\mathrm{O} \text { from the amide group } \\
\text { of proteins, }-\mathrm{C}-\mathrm{N} \text { belonging to } \\
\text { aromatic and aliphatic amines }\end{array}$ & $\mathrm{N} / \mathrm{A}$ & $\begin{array}{l}4 \text { weeks/room } \\
\text { temperature }\end{array}$ \\
\hline & spherical/4-55 nm & HRTEM/XRD/FTIR/UV-vis & $\begin{array}{l}\text { antibacterial } \\
\text { activity }\end{array}$ & {$[174]$} \\
\hline \multirow[t]{2}{*}{$\begin{array}{l}\text { Penicillium } \\
\text { chrysogenum }\end{array}$} & deionized water & $\begin{array}{l}-\mathrm{O}-\mathrm{H}, \mathrm{C}-, \mathrm{C}-\mathrm{O}-, \mathrm{COO}-\text {, and }-\mathrm{N}-\mathrm{H} \\
\text { stretching associated with proteins }\end{array}$ & $\mathrm{N} / \mathrm{A}$ & $-/ 28{ }^{\circ} \mathrm{C}$ \\
\hline & spherical/9-17.5 nm & FTIR/TEM/DLS & $\begin{array}{l}\text { antifungal activity } \\
\text { and ability to } \\
\text { prevent } \\
\text { mycotoxin } \\
\text { production }\end{array}$ & {$[172]$} \\
\hline
\end{tabular}




\begin{tabular}{|c|c|c|c|c|}
\hline \multirow[t]{2}{*}{ Fusarium oxysporum } & $\begin{array}{l}\text { deionized water } \\
\text { spherical/1-50 nm }\end{array}$ & $\begin{array}{l}\text { not mentioned } \\
\text { SEM/TEM }\end{array}$ & $\begin{array}{l}\mathrm{N} / \mathrm{A} \\
\text { antibacterial } \\
\text { activity }\end{array}$ & $\begin{array}{l}-/ 37^{\circ} \mathrm{C} \\
{[171]}\end{array}$ \\
\hline & \multicolumn{3}{|c|}{ Sugars } & \\
\hline \multirow[t]{2}{*}{ brown sugar } & distilled water & $\begin{array}{l}\mathrm{C}-\mathrm{C} \text { and } \mathrm{C}-\mathrm{O} \text { corresponding to } \\
\text { sucrose, glucose and fructose }\end{array}$ & $N / A$ & $2 \mathrm{~h} / 50^{\circ} \mathrm{C}$ \\
\hline & $\begin{array}{l}\text { varied (spheres, cubes, } \\
\text { and bars)/- }\end{array}$ & UV-vis/TEM/HRTEM/EDX/FTIR/MS & $\begin{array}{l}\text { biomedical and } \\
\text { pharmaceutical } \\
\text { applications }\end{array}$ & {$[199]$} \\
\hline \multirow[t]{2}{*}{ white sugar } & double-distilled water & $\begin{array}{l}\text {-OH groups corresponding to } \\
\text { gluconic acid }\end{array}$ & $\mathrm{N} / \mathrm{A}$ & $\begin{array}{l}10 \text { min under } \\
\text { sunlight, } 2-3 \mathrm{~h} \\
\text { without } \\
\text { sunlight/room } \\
\text { temperature }\end{array}$ \\
\hline & spherical/10-25 nm & UV-vis/FTIR/NTA/TEM & $\begin{array}{l}\text { biomedical and } \\
\text { pharmaceutical } \\
\text { applications }\end{array}$ & {$[200]$} \\
\hline
\end{tabular}

potential in targeted imaging and therapeutic delivery systems [272]. In addition, they can be used to synthesize 1D Ag nanostructures $[262,263]$. This characteristic is obtained due to the rod-shape morphology of some plant viruses such as TMV. The synthesis of AgNPs using 1D templates can facilitate their application as bio-semiconductors [263]. One of the main advantages of viral templates is the simple synthesis of small AgNPs [261,262]. However, their major drawback is the lack of strong metal-binding sites along the biotemplate surface [262]. In addition to that, the preparation of the viral templates is timeconsuming and multiple coating cycles may be required to yield a uniform coating of metal nanostructures on their surface. We have recently reviewed engineered TMV and its virus-like-particles (VLPs) for synthesis of biotemplated nanomaterials. We also discussed the recent advances on novel barely stripe mosaic virus (BSMV) and its VLP as a novel template for metal nanoparticle synthesis [273,274].

\subsection{Algae-mediated synthesis}

The synthesis of AgNPs using marine-based microorganisms has emerged as one of the novel and promising routes due to their non-toxicity and eco-friendly nature $[189,275]$. Cyanobacteria, brown algae, and green algae are the most common types of algae used for the synthesis of AgNPs [189]. For most applications, the single-cell type of the organism known as microalgae is used to synthesize AgNPs [191]. This enables formation of homogeneous microalgal suspensions which can be used directly for the synthesis process. In the synthesis process, the microalgal biomass in the aqueous phase, cell-free aqueous extract, aqueous supernatant of dried algae, or aqueous filtrate of the broth are mixed with the silver solution (mostly silver nitrate) to synthesize AgNPs [189]. The synthesis process is intracellular when the reaction takes place within the cells, and extracellular when biosynthesis occurs outside of cells due to the presence of biomolecules [191], which depends on the type of cell culture used. For instance, cell-wall deficient cells are typically more inclined towards intracellular biosynthesis as the cell-wall is known to act as a barrier for the diffusion of metal cations into the cytoplasm [276]. When synthesized, the AgNPs are capped by a matrix of polysaccharides in and out of the cells [191], and the size of synthesized AgNPs varies depending on the cell type; however, AgNPs with average sizes as low as $4.3 \mathrm{~nm}$ [192], and as high as $35 \mathrm{~nm}$ [277] were reported. The synthesized AgNPs are typically spherical in shape [192,278,279]; however, varied morphologies were also reported [193]. Among the applications, algae-mediated AgNPs have shown effective antioxidant and antibacterial activity [264,275]. The advantages of the algae-mediated synthesis process are low reaction temperatures, use of non-hazardous reagents, and synthesis of relatively small particles with uniform morphology. However, the main disadvantage of this type of biosynthesis is the significantly low production rate [264,265].

\subsection{Plant/plant-extract-mediated synthesis}

The synthesis of Ag nanostructures using plants and plant extracts has recently gained more attention. These methods can act as appropriate alternatives for other methods as a result of their simplicity, low-cost, non-toxicity, and simple scale-up capability [186]. In addition, thanks to their non-pathogenic and biocompatibility characteristics, AgNPs synthesized by plants and plant extracts are ideal for biomedical applications [304]. 
Plant extracts contain phenolic compounds such as flavonoids and alkaloids which are soluble in water [305]. These compounds provide the reagent with unique reducing and capping characteristics [306]. This can be proved by FTIR observations, where polyphenols are a major common functional group responsible for reduction of $\mathrm{Ag}$ ions and stabilization of AgNPs (Table 2). The AgNPs can be functionalized with respect to the type of plant or plant extract reagent and reaction conditions [307]. Plants are a natural source for the removal of heavy metals from soils and underground water [308]. The removal of heavy metals takes place by phytoremediation strategies such as phytoextraction, phytofiltration, phytostabilization, phytovolatilization, phytodegradation, rhizodegradation, and phytodesalination [309]. A variety of plants such as Noccaea caerulescens, Pteris vittata, and Sedum plumbizincicola were previously demonstrated to have substantial heavy-metal detoxification capability [310-312]. One of the most significant factors in the detoxification process is the redox potential [313], which is seen as an opportunity to utilize plants and their components for the reduction of metal cations and synthesis of metal nanoparticles such as AgNPs. The synthesis of AgNPs by plants and their components may be categorized into in vivo and in vitro synthesis processes [140]. The in vivo synthesis refers to synthesis of AgNPs inside the plants and in vitro synthesis refers to synthesis by components extracted from the plants. In the first in vivo synthesis study, Torresdey et al. [314] synthesized AgNPs (spherical, 2-20 nm in diameter) using Alfalfa Sprouts, where it was reported that silver $\left(\mathrm{Ag}^{0}\right)$ was absorbed from the agar medium through the roots and transferred into plant shoots. It was also reported in this work that the nucleation and formation of AgNPs occur within the plant tissue. However, it is debated by other studies whether the $\mathrm{Ag}^{+}$ ions are reduced outside or inside of the plant [140,315]. Later, several studies reported the synthesis of AgNPs using Brassica Juncea [316-319], in which they reported the presence of AgNPs in plant biomass. It is shown that the major compounds for the synthesis of AgNPs are phytochemicals that naturally exist in plants including flavones, terpenoids, catechins, and polyphenols [183,315,320], which may also include carboxylic acids, ketones, and aldehydes functional groups. Many studies have reported synthesis of AgNPs by in-plant phytochemicals in the water-soluble form [321]. This is advantageous because the water-solubility of the phytochemical compounds simplifies the process. Various parts of plants such as roots, fruits, seeds, needles, and aerial parts may be used for extraction of phytochemicals [321]. These extracts contain a substantial amount of polyphenols which are strong antioxidants $[140,186]$ with significant redox potential. A comprehensive mechanism for the synthesis of AgNPs by plant extracts has not yet been proposed; however, the reduction mechanism may be explained by identifying the responsible functional groups. Makarov et al. [322] hypothesized that when flavonoids, a large family of polyphenols, are used as reducing agents, reactive hydrogens are released via a tautomerization process, in which flavonoids are transformed to the keto-form, which leads to reduction of $\mathrm{Ag}^{+}$ to $\mathrm{Ag}^{0}$. In addition, hydroxy $(-\mathrm{OH})$ groups are known to be a prominent functional group for reduction of $\mathrm{Ag}^{+}$[321]. The concentration of the polyphenols varies depending on the plant from which they were extracted [321]. Therefore, the properties of the synthesized AgNPs, such as size and morphology, can be tuned by selecting the plant source, and adjustment of extract composition [180,323].

The plant-mediated synthesis of AgNPs is fairly simple. The reducing agents used for the synthesis of AgNPs are used in the form of soluble powder which was previously extracted, or obtained through a common extraction procedure [183]. For synthesis, the extract solution is simply mixed in an aqueous environment with the silver precursor such as $\mathrm{AgNO}_{3}$ and maintained at a desired temperature. A prominent advantage is that these reactions can be performed at room temperature, and successfully result in formation of AgNPs, which is reported by many studies [187,287,289,292-294,297,298,300]. However, the characteristics of the AgNPs, such as size and morphology, are highly sensitive to the reaction conditions. Parameters that dictate such characteristics are extract composition and concentration, temperature, $\mathrm{pH}, \mathrm{Ag}^{+}$concentration, reaction duration, and stirring rate [183].

The number of studies using plant extracts as reducing agents has been recently growing and various types of plant extracts have been used as reducing, capping, and stabilizing agents for the synthesis of AgNPs. Johnson et al. [324] synthesized AgNPs using Odontosoria Chinensis extract and reported the formation of spherical AgNPs with diameters ranging from 22.3 to $48.2 \mathrm{~nm}$. The reaction occurred at $40{ }^{\circ} \mathrm{C}$, and a complete reduction of $\mathrm{Ag}^{+}$after $10 \mathrm{~min}$ was reported. Agglomeration was observed in the case of temperatures higher than $40{ }^{\circ} \mathrm{C}$. Also, the most optimal concentration ratio was reported to be at 1:9 (extract/ $\mathrm{AgNO}_{3}$ ). They reported that carboxylic acids and hydroxy functional groups were mainly responsible for the synthesis of AgNPs, and the presence of terpenoids, tannins, polyphenols, and steroids in Odontosoria Chinensis extract was confirmed before the synthesis experiments. The synthesized AgNPs demonstrated successful anti-inflammatory and antidiabetic activities, which was due to polyphenols, terpenoids, and tannins in the extract in addition to the AgNPs themselves. Sivakumar et al. [325] synthesized spherical AgNPs with an average size of $10.3 \pm 1.7 \mathrm{~nm}$ using Parthenium hysterophorus aqueous extract. The aqueous extract was mixed with a $10 \mathrm{mM}$ $\mathrm{AgNO}_{3}$ solution with a volumetric ratio of 3:100. The mixture was incubated in the dark for $1 \mathrm{~h}$. FTIR characterization results 
showed the presence of polypeptides, amine, germinal methyl, and hydroxy groups as functional groups responsible for reducing $\mathrm{Ag}^{+}$ions and formation of AgNPs. The synthesized AgNPs then demonstrated significant antibacterial activity against E. coli, $P$. auriginosa, B. subtillis, S. aureus, E. feacalis, and $K$. pneumonia, and anticancer activity against HepG2 cell lines. It was later demonstrated by Nouri et al. [326] that synthesis using Mentha Aquatic can result in the formation of small AgNPs with relatively narrow size distribution. In their study, Mentha Aquatica leaf extract was mixed with $\mathrm{AgNO}_{3}$ solution at different reaction conditions. The most optimal reaction condition was reported to be at a $\mathrm{pH}$ of 9.5 , temperature of $90{ }^{\circ} \mathrm{C}$, concentration ratio of $1: 1$, and 60 min reaction time. However, they showed that ultrasonication can reduce both the reaction time and particle size. Without ultrasonication, the average size was observed to be $14 \mathrm{~nm}$, while with ultrasonication, this size was reduced to $8 \mathrm{~nm}$. In addition, the increase in ultrasonication power was proportional to the decrease in reaction time. The reaction time decreased from $60 \mathrm{~min}$ to $10 \mathrm{~min}$ by increasing the ultrasonication power from 0 to $200 \mathrm{~W}$. This was attributed to the enhanced diffusion of compounds due to sonic waves. In another study, Tanase et al. [327] synthesized spherical AgNPs using aqueous bark Picea abies L. extract. The reaction was performed at $70{ }^{\circ} \mathrm{C}$ and $\mathrm{pH} 9$ with different $\mathrm{AgNO}_{3}$ and extract concentrations. Although colloidal AgNPs were synthesized, the size and size distribution were significantly high $(100-500 \mathrm{~nm})$. The most optimal scenario was attributed to $1 \mathrm{mM}$ of $\mathrm{AgNO}_{3}$ with a volumetric ratio of 1:10 (extract $/ \mathrm{AgNO}_{3}$ ) and a reaction time of $3 \mathrm{~h}$. The results demonstrated that higher $\mathrm{AgNO}_{3}$ concentration results in inhibition of the synthesis process. The responsible functional groups were confirmed by FTIR to be aldehydic, carbonyl, and hydroxy groups of phenols and carboxylic acids. The AgNPs showed strong antibacterial activity against $E$. coli and Klebsiella pneumonia, comparable to the antibacterial activity of gentamicin.

Most plant extract synthesis studies use extracts as both reducing and capping agents at the same time. However, this might not be advantageous in some cases. Ranoszek-Soliwoda et al. [188] synthesized spherical AgNPs using cacao beans and grape seed extract and investigated the effect of sodium citrate with various molar ratios with respect to $\mathrm{AgNO}_{3}$ solution (1.5-6 for sodium citrate, and 1.5-9 for the extract). Acetone and ethanol were used as a solvent for cacao beans and grape seed extract, respectively. The initial concentration of both reagents was set to $2 \mathrm{wt} \%$, and the concentration of $\mathrm{AgNO}_{3}$ was kept constant at $1 \mathrm{wt} \%$. The mixtures were heated for $15 \mathrm{~min}$ and then cooled down to room temperature. Using various molar ratios, they reported that cacao bean extract resulted in aggregation of AgNPs followed by sedimentation, and therefore, it cannot be used as reducing and stabilizing agents at the same time. However, monodisperse AgNPs with an average size of approximately $15 \mathrm{~nm}$ were obtained using sodium citrate as the capping agent. Under these conditions, the molar ratio did not have a significant effect on the size of AgNPs, and their size was maintained between 11-15 $\mathrm{nm}$ at different molar ratios of cacao bean extract and sodium citrate. The reason was attributed to the fact that sodium citrate is able to form stable complexes with the polyphenols present in the extract, which then acts as reducing and capping agent. This was nevertheless different for grape seed extract. Without the presence of sodium citrate, AgNPs agglomerated and had a large size distribution, but in the presence of sodium citrate, the size was maintained at 10-18 $\mathrm{nm}$ for different sodium citrate molar ratios, and the size distribution was smaller. Furthermore, it was reported by Mohaghegh et al. [328] that sodium citrate can form complexes with $\mathrm{Ag}^{+}$at ambient temperature, and act as reducing and capping agent at the elevated temperature. In another study, Ranoszek-Soliwoda et al. [329] reported the formation of citrate-tannic acid complexes which were responsible for reduction and stabilization of tannic-acid-mediated AgNPs. They also stated that the mere use of sodium citrate could result in inhomogeneous sizes and a broad size distribution. To the best of our knowledge, it has not yet been well determined what type of plant extracts can be used as reducing and capping agents at the same time. The formation of silver nanoparticles by plant extracts depends highly on the plant extract composition along with various process parameters such as $\mathrm{pH}$, temperature, and concentration ratio.

3.5.1 Effect of $\mathbf{p H}$. The $\mathrm{pH}$ is known to be a significant parameter in controlling the size, morphology, and stability of AgNPs [330]. Singh et al. [331] synthesized spherical AgNPs using Hibiscus leaf extract ranging $12-17 \mathrm{~nm}$ in diameter. Temperature and $\mathrm{pH}$ were reported to be the most significant factors in controlling the synthesis process. The effect of $\mathrm{pH}$ was mainly investigated in this work and it was observed that at $\mathrm{pH} 3$ the AgNP formation process stops as there was not any further color change, and at pH 10 agglomeration occurs upon the addition of the $\mathrm{AgNO}_{3}$ solution. At pH 6 however, the reduction process started upon the addition of $\mathrm{AgNO}_{3}$ and was observed for $30 \mathrm{~min}$. The most optimal condition to minimize the size of AgNPs was reported to be at a $\mathrm{pH}$ of 6 , temperature of $70^{\circ} \mathrm{C}$, $30 \mathrm{~min}$ of reaction time, and $5 \mathrm{mM} \mathrm{AgNO}_{3}$ solution (1:1.5 volumetric ratio with respect to the extract). Their FTIR studies demonstrated that the hydroxy functional groups and polysaccharides were responsible for reduction and stabilization of AgNPs. In another work by Handayani et al. [332], it was demonstrated that alkaline conditions are more favorable for the synthesis of smaller nanoparticles. They synthesized AgNPs using Pometia pinnata (Matoa) leaf extract for $24 \mathrm{~h}$ with 1:2 
volumetric ratio (extract/ $\mathrm{AgNO}_{3}$ ). They demonstrated that at $\mathrm{pH} 11$, the AgNPs were 10-50 nm in size and of spherical and hexagonal morphologies, while at $\mathrm{pH} 4$ the AgNPs were observed to be $50-80 \mathrm{~nm}$ with spherical and triangular morphologies. It was also reported that a change in $\mathrm{pH}$ affects the reducing capability of the extract, which is also confirmed by other studies [333,334]. The $\mathrm{pH}$ is also reported to control the zeta potential $[330,332]$ and results in particle stability adjustments [330]. The higher the value of the zeta potential, the stronger the long-term stability of the AgNPs, thereby preventing their agglomeration [330,335]. Alkaline $\mathrm{pH}$ also has the potential of increasing the reaction rate due to the enhanced deprotonation of phenolic compounds in basic condition [336]. In addition, alkaline $\mathrm{pH}$ enables more $-\mathrm{OH}$ to take part in the reduction reaction, thus increasing the reduction strength [337].

3.5.2 Effect of temperature. Temperature is another crucial factor in controlling the size and morphology of AgNPs. Increasing the temperature equals increasing the reaction rate which is favorable for rapid synthesis. However, high temperatures (more than $60{ }^{\circ} \mathrm{C}$ ) may result in denaturation of the extract compound, and thus alter their reduction potential and result in a significant increase in particle size and even agglomeration [140]. The extract compound should therefore be carefully selected and tested in order to avoid denaturation. Madivoli et al. [338] synthesized AgNPs using Lantana trifolia extract by changing the temperature from 20 to $35^{\circ} \mathrm{C}$. It was shown that a decrease in temperature resulted in a broader surface plasmon peak, attributing to a larger size distribution contrary to higher temperatures. However, increasing the temperature resulted in the formation of larger particles, $60 \mathrm{~nm}$ in the case of $35^{\circ} \mathrm{C}$ and $48 \mathrm{~nm}$ in the case of $20^{\circ} \mathrm{C}$. Nevertheless, AgNPs with a size of $37 \mathrm{~nm}$ were obtained at $30{ }^{\circ} \mathrm{C}$. Therefore, there was a sharp increase in particle size by changing the temperature from 30 to $35{ }^{\circ} \mathrm{C}$. In addition, in a study performed by Anbu et al. [339], AgNPs were synthesized at $50^{\circ} \mathrm{C}$ and were slightly larger compared to those synthesized at $37^{\circ} \mathrm{C}$. The increase in particle size due to an increase in temperature was also confirmed by previous studies [307,340-342]. Furthermore, Liu et al. [343] used Cinnamomum Camphor leaf extract to synthesize AgNPs. They demonstrated that an increase in temperature will result in an increase in particle size under sufficient $\mathrm{Ag}^{+}$ions $\mathrm{Ag}^{+}$concentration equivalent or excess to the extract concentration), and results in a decrease in particle size under insufficient $\mathrm{Ag}^{+}$ ions (extract concentration excess to $\mathrm{Ag}^{+}$concentration). They concluded that under sufficient $\mathrm{Ag}^{+}$ions, particle size is not affected by nucleation, but rather by growth. They proposed on the contrary, that under insufficient $\mathrm{Ag}^{+}$concentration, all the $\mathrm{Ag}^{+}$ions are consumed rapidly through a burst nucleation process, thus not allowing further growth. It can therefore be concluded that temperature and concentration are both signifi- cant factors and have an interactive effect on controlling the morphology and size of AgNPs.

3.5.3 Effect of concentration/concentration ratio. The concentration of plant extract and silver precursor, as well as their ratio, play a major role in controlling the size and morphology of AgNPs. Hasnain et al. [344] synthesized spherical AgNPs using purple heart plant leaf extract and investigated the effect of various parameters using the design of experiments (DoE) approach. Their results indicated that an increase in $\mathrm{AgNO}_{3}$ concentration (from 0.01 to $0.1 \mathrm{M}$ ) at constant purple heart plant extract volume $(1.4 \mathrm{~mL})$ and relatively lower temperatures $\left(60^{\circ} \mathrm{C}\right)$, resulted in a decrease in particle size (from $\approx 120$ to $100 \mathrm{~nm}$ ). They also observed an initial decrease (from $\approx 310$ to $150 \mathrm{~nm}$ ) followed by a sharp increase in particle size (from $\approx 150$ to $335 \mathrm{~nm}$ ) at higher temperatures $\left(80^{\circ} \mathrm{C}\right.$ ). In addition, an increase in $\mathrm{AgNO}_{3}$ concentration at constant temperature $\left(80^{\circ} \mathrm{C}\right)$, and relative to various volumes of purple heart plant extract, resulted in an initial decrease (from $\approx 310$ to $150 \mathrm{~nm}$ ) followed by an increase in particle size (from $\approx 150$ to $>350 \mathrm{~nm}$ ). However, changing the purple heart plant extract volume did not have a significant effect on the particle size. Therefore, the $\mathrm{AgNO}_{3}$ concentration had a much greater effect on determining particle size. Also changing the temperature from 60 to $80{ }^{\circ} \mathrm{C}$ resulted in an approximately linear and continuous increase in particle size (from $\approx 100$ to $330 \mathrm{~nm}$ ), meaning that temperature was a stronger parameter compared to plant extract concentration. Furthermore, Htwe et al. [345] synthesized AgNPs with quasi-spherical morphology using Imperata cylindrical plant extract as the reducing agent and ascorbic acid as the capping agent. They showed that increasing the $\mathrm{AgNO}_{3}$ concentration (from 0.5 to $0.9 \mathrm{mM}$ ), at constant extract and ascorbic acid concentration, resulted in increasing the particle size (from 32.7 to $39.9 \mathrm{~nm}$ ). Their ultraviolet-visible (UV-vis) spectroscopy results also showed that at $0.5 \mathrm{mM} \mathrm{AgNO}_{3}$, the absorption peak was narrower compared to 0.7 and $0.9 \mathrm{mM}$ $\mathrm{AgNO}_{3}$, indicating lower size distribution. Khan et al. [346] utilized Piper Betle leaf aqueous extract to synthesize AgNPs. They investigated the effect of concentration on the size and stability of $\mathrm{AgNPs}$ by testing various $\mathrm{AgNO}_{3}$ concentrations (1, $2,3$, and $4 \mathrm{mM})$ and extract concentrations (1:2, 1:4, and 1:8 dilution ratio with respect to the crude extract). They reported the most optimal conditions to be at $2 \mathrm{mM}$ of $\mathrm{AgNO}_{3}$ and 1:4 dilution ratio of the extract. Their results indicated that increasing $\mathrm{AgNO}_{3}$ concentration leads to shifting the UV-vis peak to the higher wavelengths indicated formation of larger nanoparticles. Aggregation was also observed for higher $\mathrm{AgNO}_{3}$ concentrations. They also reported that higher concentrations of the extract could result in instability and aggregation of AgNPs, which is also confirmed by a previous study [347]. 


\section{One-dimensional silver nanostructures and their green synthesis}

One-dimensional (1D) silver nanostructures are characterized as a collection of silver atoms in a one-dimensional pattern. They range from tens to a few hundreds of nanometers in diameter, and their length is of the order of tens of micrometers. 1D silver nanostructures could either be silver nanowires (AgNWs) or silver nanorods (AgNRs). These two are distinguished by the aspect ratio, which is the ratio of the length to the diameter. Typically, 1D silver nanostructures with an aspect ratio of 10 or higher are considered as AgNWs while those with aspect ratios lower than 10 are considered as AgNRs [90]. In general, 1D metallic nanostructures have unique plasmonic properties as they can uniquely interact with light because of their plasmonic characteristics [348,349]. AgNWs, in particular, possess great surface plasmon resonance (SPR) characteristics, high conductivity, and remarkable flexibility in addition to having a simple fabrication process [350]. The synthesis of AgNWs has been under progressive investigation during the last decade due to their broad range of applications including nanoelectronics.

AgNWs have been widely applied in electronics. In particular, there have been promising developments in transparent conductive electrode (TCE) manufacturing for next generation touchscreens by applying AgNWs [351-354]. To name a few, $\mathrm{C} 3 \mathrm{Nano}^{\circledR}{ }^{\circledR}$, Cambrios ${ }^{\circledR}$, and $3 \mathrm{M}^{\circledR}$ are among the companies that have been developing AgNW-based inks for TCEs used in the flexible touchscreens manufacturing. Currently, indium tin oxide (ITO) is still the dominant material used in TCEs due to its high transmittance and low sheet resistance [67,355]. Its high conductivity and transmittance level made ITO a fairly appropriate candidate for TCEs; however, weak flexibility, limited supply, costly coating process, highly expensive large-scale manufacturing, and the cost of the material itself have limited a potentially broader application in optoelectronics $[67,90]$. As a result, ITO-embedded TCEs are gradually giving their position to AgNW-embedded TCEs. One of the most important aspects of the synthesis of AgNWs is to precisely control their size and morphology to achieve a uniform high aspect ratio that strongly affects their optical and transmittance properties [356-358] Achieving a uniform high aspect ratio depends on the synthesis process and the reaction conditions [357]. As a result, selection of the reducing and stabilizing agents, and identifying the most important factors that control the synthesis process are essential in determining the length and diameter of the synthesized AgNWs.

There are generally two methods for the synthesis of AgNWs, which are hard template and soft template methods. In hard template methods, AgNWs are produced using cylindrical nanoporous structures such as carbon nanotubes (CNTs) [359].
On the other hand, in soft template methods, capping agents such as polyvinylpyrrolidone (PVP) or cetyltrimethylammonium (CTAB) are used to direct the growth of initially formed silver seeds to AgNWs [349]. Hard template methods have several disadvantages compared to soft template ones. The yield of AgNWs is significantly low and the templates are very hard to remove [90]. On the other hand, the template removal issue is nonexistent in soft template methods and soft templates can be used in the solution phase. Block copolymers [360] and polyvinyl alcohol (PVA) [361] are among the other soft templates used for the synthesis of AgNWs. Currently, the most common method for the synthesis of AgNWs is through the polyol process, in which ethylene glycol is usually used as the solvent and reducing agent, PVP as the capping agent, and in the presence of a small amount of salt mediator. This method is being used to synthesize AgNWs in large commercial scales and has proved to be simple and able to provide relatively proper control over morphology and aspect ratio [362]. The synthesis of AgNWs by the polyol approach was first introduced in 2002 by Xia's group [363]. In early studies, the synthesis was performed using Pt seeds to promote nucleation and growth of AgNWs. In order to make the process more economical and simpler, while maintaining a high yield and aspect ratio, further modifications were later applied in the synthesis process, such as the gradual addition of $\mathrm{AgNO}_{3}$ solution using syringe pumps [364], using salt mediators such as Fe [365] and $\mathrm{Cu}$ [366] metal salts, and increasing preheating time [357]. The combination of exceptional size and morphology-dependent chemical, optical, and physical characteristics of 1D Ag nanostructures with their potential antibacterial activity, is evidence of a critical need to develop synthesis processes appropriate for their industrial-scale applications. These synthesis processes must meet some criteria including being scalable, low cost, and low toxicity of the materials involved in the synthesis process. These criteria are in excellent agreement with the goals in the development of green and sustainable synthesis of metal nanostructures. Polyol synthesis has a fairly high energy consumption due to high temperatures $\left(>120^{\circ} \mathrm{C}\right)$. In addition, in a more recent polyol processes, a $1 \mathrm{~h}$ preheating time for ethylene glycol was required, which adds to the experiment duration at relatively high temperatures. While progress with improving the polyol process is ongoing, some studies started to use green reagents for the synthesis of AgNWs. We have recently reviewed the polyol silver nanowire synthesis and its outlook for a green process [367]. In 2009, Tian et al. synthesized single-crystalline AgNWs $-25 \mathrm{~nm}$ in diameter and $20 \mu \mathrm{m}$ in length - using tannin acting as both reducing and capping agent [368]. Tannin is a natural polyphenolic compound found in various plant extracts, such as oak barks, galls, and teas. They can act as strong reducing agents due to the oxidization of $-\mathrm{OH}$ functional groups in their molecular structure [369]. The 
AgNWs were synthesized at room temperature, and it was found that an increase in the tannin concentration resulted in AgNWs with a lower aspect ratio. They reported that the slow reduction rate could favor the formation of twinned silver seeds with decahedron morphology. On the contrary, in a more recent work, Dong et al. reported that at low temperatures, tannin molecules cannot selectively adsorb on the $\{100\}$ facets of AgNWs due to the lack of driving energy [291]. In this work, AgNWs were synthesized at $50{ }^{\circ} \mathrm{C}$ for $2 \mathrm{~h}$ with the same $\mathrm{AgNO}_{3}$ and tannin concentrations used in Tian et al.'s study. The yield of AgNWs in this work was reported to be much higher (as high as 90\%) compared to Tian et al.'s study (5-10\%). The AgNWs were reported to be $60 \mathrm{~nm}$ thick in diameter and several tens of micrometers long. Although low temperatures were considered to be unfavorable for the growth of AgNWs, higher temperatures (higher than $50{ }^{\circ} \mathrm{C}$ ) were not favorable either, which is due to the fact that tannin molecules do not have adequate time to adsorb on the specific facets, thus resulting in formation of nanoparticles. They also reported that decreasing the $\mathrm{pH}$ from the basic to acidic region results in increasing the yield of AgNWs, with pH 5 being the optimal value. In another study, Lin et al. synthesized AgNWs using Cassia fistula as both reducing and capping agent [370]. The reaction was performed at room temperature for $48 \mathrm{~h}$, and the synthesized AgNWs were reported to be $50-60 \mathrm{~nm}$ in diameter and tens of micrometers in length. They reported that the Ostwald ripening process was indispensable to the accumulation of smaller nanoparticles into AgNRs and ultimately AgNWs. They also reported that increasing the temperature would result in the formation of mere nanoparticles due to the alteration of interaction between the biomolecules and certain facets. Jeevika et al. used clove oil, as both reducing and capping agent to synthesize AgNWs with a diameter of $39 \mathrm{~nm}$ and length of $3 \mu \mathrm{m}$ [292]. Clove oil is rich in eugenol, which is a phenolic compound. The reaction was carried out at room temperature and $-\mathrm{OH}$ functional groups from eugenol were found to be responsible for the synthesis of AgNWs. Eugenol molecules were also responsible for stabilizing $\mathrm{AgNWs}$, as they can bind to the initially formed $\mathrm{Ag}$ seeds' facets and direct the growth. Soleimani et al. synthesized AgNRs with sharp and blunt ends using $F$. oxysporum as a reducing agent and starch as a capping agent [371]. In their work, the reaction was carried out at $30{ }^{\circ} \mathrm{C}$ for 3 days, with the $\mathrm{pH}$ adjusted at 3. For AgNRs with sharp ends, the average diameter and length were $181 \mathrm{~nm}$ and $4.3 \mu \mathrm{m}$, and for AgNRs with blunt ends, the average diameter and length were $204 \mathrm{~nm}$ and $3.9 \mu \mathrm{m}$, respectively. They reported that the initially formed Ag nucleus was single-crystal, and starch was able to cover the $\{100\}$ facets of the NRs. It is also noteworthy to mention that other morphologies were obtained by changing the concentration of $\mathrm{AgNO}_{3}$, temperature, $\mathrm{pH}$, and reaction time. In another study, Flores-González et al. used Camellia Sinensis extract in presence of a small concentration of PVP to synthesize AgNWs with dimensions of $50 \mathrm{~nm}$ in diameter and $1.3 \mu \mathrm{m}$ in length [287]. Camellia Sinensis is a strong phenolic compound with significant antioxidant potential. The reaction was carried out at room temperature for 30 days under dark conditions. They reported that PVP was able to selectively adsorb to $\{100\}$ and $\{111\}$ facets, thus directing the growth of AgNWs. They also explained that the Ostwald ripening process resulted in attraction of the previously formed AgNPs, therefore leading to NW growth. However, it is important to note that at the beginning of the reaction, PVP was added to the reaction in the form of powder, and the temperature of the solution was kept at $45{ }^{\circ} \mathrm{C}$ for complete dissolution of the PVP. Nadagouda et al. used vitamin B2 to synthesize AgNWs and AgNRs at room temperature for $24 \mathrm{~h} \mathrm{[204].} \mathrm{In} \mathrm{their} \mathrm{study,} \mathrm{AgNWs} \mathrm{with} \mathrm{diameters}$ ranging from 10 to $20 \mathrm{~nm}$ and lengths of several hundreds of micrometers were obtained when isopropanol was used as the solvent, and AgNRs structures were obtained with a thickness of 100 to $200 \mathrm{~nm}$ and lengths of tens of micrometers when acetone and acetonitrile were used as the solvent. Hemmati et al. obtained AgNRs using brown sugar as both reducing and capping agent [199]. The AgNRs were around $50 \mathrm{~nm}$ thick in diameter and a few micrometers long. The reaction was performed at $50{ }^{\circ} \mathrm{C}$ for $2 \mathrm{~h}$.

The main advantage of the green synthesis of 1D Ag nanostructures is the fact that the selected green reagent can act as both capping and reducing agent at the same time, therefore triggering and continuing the reduction process while controlling growth. As a result, providing an additional capping agent such as PVP may not be required, which could reduce the overall material cost, since green reducing agents such as plant extracts are not expensive. Another important advantage is the possibility of performing the synthesis experiments at significantly lower temperatures compared to the polyol process, where temperatures of $120-200{ }^{\circ} \mathrm{C}$ are required. Some reactions can even be carried out at room temperature. This is quite beneficial in reducing energy consumption and risk, thus making the largescale synthesis processes more cost-effective and safer. Another advantage is the fact that synthesis experiments may be performed without using exotic seeds and metal salts. Green reagents such as tannin and clove oil have shown that the synthesis of AgNWs with high yield and aspect ratio is possible. As a result, if the responsible factors are identified properly, high aspect ratio and yield can be achieved, comparable to those achieved through the polyol process.

\section{Effect of reaction parameters on the green synthesis of $1 \mathrm{D} \mathrm{Ag}$ nanostructures}

The effect of reaction parameters such as $\mathrm{pH}$, temperature, reducing agent concentration, and silver precursor concentra- 
tion is crucial for the formation of 1D silver nanostructures such as AgNRs and AgNWs. To facilitate the growth of 1D nanostructures, the reaction conditions should be favorable to initiate an anisotropic growth of the seeds in a kinetically controlled synthesis process by adjusting and controlling the generation and deposition rate of silver atoms [372]. When the number of atoms for heterogeneous nucleation is lower than the growth sites available on a seed surface, the seed is expected to undergo an anisotropic growth [373]. There are different ways to make this process possible. For instance, applying a relatively low temperature (i.e. room temperature) can result in the suppression of adatomic diffusion, and consequently results in an anisotropic growth process [373]. However, this also depends on the reducing capability of the reagent at room temperature as well as its concentration, reaction $\mathrm{pH}$, and metal precursor concentration. For instance, tannic acid, which is a hydrolysable form of tannin, has been used for the synthesis of both AgNPs and AgNWs [368,374,375]. Tannic acid has the potential to be hydrolyzed into glucose and gallic acid in weak basic/acidic conditions at room temperature [376,377]. Gallic acid can serve as a reducing agent while glucose serves as a stabilizing agent [378]. This promising dual property of tannic acid makes it usable as both reducing and capping agent. The reducing capability of gallic acid increases by increasing the $\mathrm{pH}$ [378]. In the case of AgNP synthesis at room temperature and basic $\mathrm{pH}$ using tannic acid, gallic acid plays its role as a strong reducing agent while glucose acts as a strong stabilizing agent [379,380]. However, in the case of AgNW or AgNR synthesis at room temperature using tannic acid, the acidic $\mathrm{pH}$ is considered to be more favorable due to the slower reduction rate provided by gallic acid [375]. Three studies agree on the fact that acidic $\mathrm{pH}$ is more favorable for the growth of 1D nanostructures in the presence of tannic acid [291,368,375]. However, higher temperatures may not be favorable for anisotropic growth due to highly rapid nucleation. Two studies have considered the room temperature conditions to be more favorable for the growth of AgNWs in acidic $\mathrm{pH}$ and the presence of tannic acid [368,375]. However, only one study by Dong et al. reported slightly higher temperatures $\left(50{ }^{\circ} \mathrm{C}\right)$ to be more favorable for the formation of AgNWs [291]. Although they mentioned that this is due to difference in facet absorption energy at different temperatures, a clear and deep explanation remains disputed as to why this difference occurs. The study on the effect of $\mathrm{pH}$ and temperature on the yield of 1D silver nanostructures using other green reagents remains limited.

The concentrations of the reducing agent and silver precursor are other important factors in controlling the yield of $1 \mathrm{D} \mathrm{Ag}$ nanostructures. Yi et al. investigated the effect of tannic acid concentration on the yield of 1D nanostructures by changing its concentration from 5 to $1 \mathrm{mM}$ while keeping the $\mathrm{AgNO}_{3}$ concentration at $3 \mathrm{mM}$ [375]. They demonstrated that the yield of 1D nanostructures such as nanorods increased as the tannic acid concentration decreased to $1 \mathrm{mM}$. This is also in accordance with the study by Tian et al. where they used a 1 $\mathrm{mM}$ tannic acid concentration [368]. This was attributed to the sufficiently slow reduction process for the formation of single crystal nanowires. However, the mere effect of $\mathrm{AgNO}_{3}$ concentration on the yield of 1D silver nanostructures was not investigated.

Considering the knowledge gap in the green synthesis of 1D Ag nanostructures, it is essential to expand the number of green reducing agents for the synthesis of $1 \mathrm{D} \mathrm{Ag}$ nanostructures. Moreover, future studies are expected to focus on the effect of reaction parameters on yield and aspect ratio of $1 \mathrm{D} \mathrm{Ag}$ nanostructures in these novel green and sustainable synthesis processes to not only tune the generation or deposition rate of silver atoms, but to control the surface diffusion rate of adatoms as well. Finally, due to the intrinsic instability of 1D Ag nanostructures because of their large surface to volume ratio, it is essential to manipulate the reaction conditions and parameters to preserve their 1D structure during the green synthesis process.

\section{Current state and key challenges in the green synthesis of 1D Ag nanostructures}

There are few green and sustainable methods of 1D synthesis of Ag nanostructures, where most of the syntheses are smallscale for a feasibility study. There are also inherent challenges in batch synthesis, such as challenges in batch-to-batch reproducibility. Moreover, there is a lack of fundamental understanding behind the synthesis reaction mechanism, and the functionality and responsibility of the specific compounds are not well understood. Without this fundamental knowledge, it is not possible to control and tune the size and morphology of the synthesized 1D Ag nanostructures, and there is a broad size distribution as well. By understanding the role of each functional group in the reduction, nucleation, and growth of silver nanostructures, it would be possible to predict the applicability of the other novel green reducing agents offered by nature for silver and consequently other 1D metal nanostructures synthesis.

\subsection{Inherent challenges in small-scale green synthesis of 1D silver nanostructures}

A major gap in green 1D silver nanostructure synthesis processes resides in the small-scale of their production with low efficiency and predictability, which has not been implemented beyond the bench. The reasonable control of the synthesis process of green synthesized 1D silver nanostructures is still a 
challenge in their batch synthesis along with the challenges in batch-to-batch reproducibility. It is essential to investigate the effect of all of the reaction parameters on the size and morphology of the synthesized 1D Ag nanostructures, and find the most essential parameters that predominately control the morphology and yield of 1D Ag nanostructures. Moreover, it is important to investigate not only the simultaneous effect of these parameters but also to find the optimal reaction conditions at which the yield of 1D Ag nanostructures is maximized.

\subsection{Lack of recipe in the green synthesis of $1 \mathrm{D}$ silver nanostructures}

Countless numbers of plant extracts from different parts of plants have been reported for the synthesis of silver nanoparticles. Although there are many published studies regarding the green synthesis of silver nanoparticles, there are comparatively few regarding 1D ones. Moreover, among these few studies, some of them are by the assistance of microwave or ultraviolet irradiation process and high energy requirements. Not only there are few studies, but all of them are in very small-scale reactions with low yield of $1 \mathrm{D}$ nanostructures compared to other nanostructures. This indicates a huge gap in the green synthesis of $1 \mathrm{D} \mathrm{Ag}$ nanostructures and the necessity to expand the number of green reducing agents, specifically plantbased ones offered by nature for synthesis of 1D Ag nanostructures.

\subsection{Lack of fundamental understanding behind the green synthesis of $1 \mathrm{D}$ silver nanostructures}

The concept of size, morphology, and shape control of silver nanostructures can be achieved by investigation of the growth mechanism and adjusting synthetic parameters. The advantages of utilizing different green reducing agents such as plants and plant-derived reagents for silver nanoparticle synthesis have been of interest in studies to evaluate the mechanism behind the synthesis process including uptake of the metal ions, their bioreduction, and the possible growth route for nanoparticle formation. However, there is a lack of fundamental knowledge regarding the reaction mechanism of green synthesis of $1 \mathrm{D}$ silver nanostructures, specifically environmentally friendly large-scale synthesis, a process with strong potential to overcome the challenges in chemical synthesis of 1D silver nanostructures. After finding the specific biochemical compound(s) or ingredient(s) in the green reducing agents that is responsible for the reduction of metal ions and consequent growth of nanoparticles to a desired morphology, it is essential to investigate the reaction mechanism, which would be crucial to control the reaction and consequently control the size, shape, and morphology of the synthesized 1D silver nanostructures. This would be achievable by understanding the exact route including reduc- tion, nucleation, and growth of their green and sustainable synthesis process.

\section{Lessons from green synthesis of silver nanoparticles towards green synthesis of 1D silver nanostructures}

Many green reagents can act as reducing and capping/stabilizing agent at the same time. However, this depends on the reaction mechanism and molecular structure of the reagent and its ingredients including particular functional groups such as amines, aldehydes, and carboxylates, to name a few [199]. Understanding the reaction mechanism is essential to tune and control the size and morphology-dependent properties of silver nanostructures. An important aspect in achieving 1D Ag nanostructure is identifying key factors controlling the stabilization and growth of initially formed Ag seeds. Nevertheless, a clear description of how natural organic molecules can direct the growth of AgNWs remains absent and only a few studies have discussed a possible mechanism in that regard. Fortunately, the mechanism of AgNWs synthesis via the polyol process is better understood and can be a useful guide in understanding the reduction process and diagnosis of responsible functional groups and binding mechanisms which direct the growth of 1D Ag nanostructures in a green synthesis process. In the case of polyol synthesis, PVP controls the capping/stabilizing mechanism, ethylene glycol controls the reduction process, and the temperature and the $\mathrm{pH}$ are among the factors that control the kinetics. In the polyol process, PVP binds to $\{100\}$ facets of initially formed seeds through $\mathrm{Ag}-\mathrm{O}$ bonding [199], and directs the growth through the $\{111\}$ facet (Figure 2). In fact, the adsorption on $\{100\}$ facets will be much stronger compared to $\{111\}$ facets for the growth to take place [90]. In the study performed by Lin et al., polyhydroxy components such as alkaloids, flavonoids, and polysaccharides acted as capping ligands for the growth of AgNWs [370]. Many of the mentioned components contain carbonyl groups similar to PVP, which could possibly be responsible for ligand bonding. In addition, Lin et al. stated that the Ostwald ripening process was one of the factors in growth of AgNWs, which was also reported previously in the polyol process [381]. In another study performed by Dong et al., the tannin molecules bound to four $\{100\}$ facets of AgNWs, leaving two other $\{100\}$ facets free for growth [291]. The tannin molecule also has carbonyl groups, which might have been taking part in the formation of ligand bonds. In a recent work, Villalpando et al. used Lavandula angustifolia extract to synthesize AgNWs [382]. They mentioned that some compounds such as saponins and glycosidics that are present in the extract [383], are responsible for 1D growth of nanostructures. However, Ag-ligand interactions are part of a larger chain in the synthesis process and are related to other factors such as reduction rate, complex formation, and temperature. For 


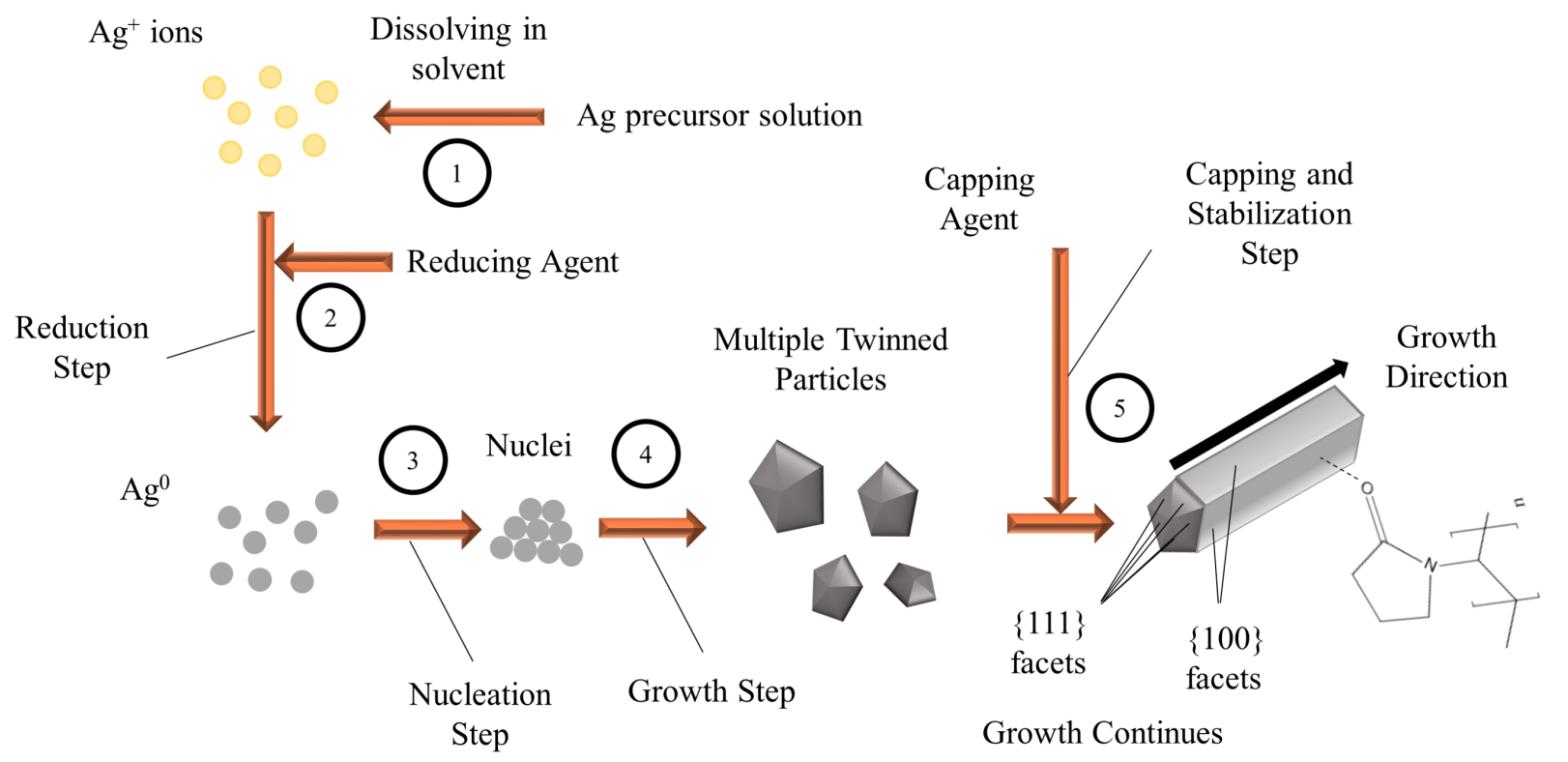

Figure 2: Synthesis steps and reaction mechanism for AgNW polyol synthesis.

instance, as mentioned earlier in the study conducted by Dong et al., higher temperatures $\left(>50{ }^{\circ} \mathrm{C}\right)$ made the conditions difficult for tannin molecules to effectively bind themselves (due to lack of adequate time) to $\{100\}$ facets of AgNWs, in which nanoparticles were formed instead of nanowires [291]. In addition, tannin molecules are able to form stable complexes with $\mathrm{Ag}^{+}$ions [369], which results in decreasing the reduction rate, paving the way for 1D anisotropic growth [384]. It was also previously mentioned that acidic $\mathrm{pH}$ is more in favor of $\mathrm{AgNW}$ formation due to moderation of the reduction rate [291,375] For instance, Dong et al. showed that the yield of AgNWs decreased by increasing the $\mathrm{pH}$ up to 9 , while the yield increased by lowering the $\mathrm{pH}$ to 5 [291]. However, further decrease resulted in a smaller yield, which was attributed to an excessive decrease in the reduction rate. Nadagouda et al. synthesized AgNRs in the presence of vitamin B2 in aqueous solution and reported that the self-assembly of AgNRs was due to strong interparticle van-der-Waals and hydrophobic interactions [204] The use of exotic seeds was therefore not needed due to the self-assembly. In addition, vitamin B2 was able to form complexes with $\mathrm{Ag}^{+}$ions and help to reduce the reaction rate, which leads to anisotropic growth of AgNRs. Wang et al. synthesized AgNWs using glucose in hydrothermal conditions $\left(180{ }^{\circ} \mathrm{C}\right.$ and under autoclave), and reported that the lower the concentration of $\mathrm{Ag}^{+}$, the more favorable the anisotropic growth of AgNWs. They added $\mathrm{NaCl}$ to the $\mathrm{AgNO}_{3}$ solution in order to form $\mathrm{AgCl}$ colloids. Due to the low solubility of $\mathrm{AgCl}$ in water, the concentration of $\mathrm{Ag}^{+}$is low at $180{ }^{\circ} \mathrm{C}$, which was favorable to growth of AgNWs. On the contrary, AgNPs were formed when only $\mathrm{AgNO}_{3}$ was used. In the latter condition, the reduction rate of $\mathrm{Ag}^{+}$ions is higher, which does not allow growth of AgNWs. Another important factor is the diffusion of initially formed seeds. Villalpando et al. recently reported that centrifugation is able to enhance the diffusion rate and therefore favor the growth of AgNWs [382]. They reported that AgNW growth is affected by coalescence and interparticle diffusion mechanism as a result of a secondary nucleation process, where smaller particles are coalesced into forming AgNWs, as described previously [385]. The enhancement of diffusion and growth of AgNWs using magnetic stirring was also reported previously [386].

\section{Conclusion}

The synthesis process is important to determine the size and morphology of $\mathrm{Ag}$ nanostructures. The green procedures for the synthesis of silver nanostructures in addition to the most common chemical and physical methods were reviewed. The advantages of green synthesis methodologies, such as their low cost, low energy consumption, scale-up capability, and simplicity that outweighs their disadvantages compared to the chemical and physical synthesis methodologies were discussed. Green synthesis processes were classified among which synthesis of Ag nanostructures using plant extracts are considered to be a promising route due to their simple utilization, aqueous nature, and non-toxic properties. Plant extracts are likely to contain certain natural compounds such as polyphenols, 
flavonoids, alkaloids, and different functional groups such as hydroxy groups and carboxylic acids, which can provide strong reducing and capping capability. Due to their broad scope, plant extracts are used for preparation of various Ag nanostructures with different sizes, and the literature studies on this subject have been growing during the last decade. The different green reagents based on studies in the literature at different reaction conditions to synthesize Ag nanostructures with different sizes and morphologies were outlined. In addition to reagent type, the synthesis of Ag nanostructures using plant extracts is highly dependent on various process factors such as temperature, reagent concentration, and $\mathrm{pH}$ that were discussed. The green synthesis of 1D Ag nanostructures using plant extracts is gaining more attention and various compounds such as tannins, glucose, clove oil, and other natural extracts may be used as both reducing and capping agents to synthesize 1D Ag nanostructures. Unlike the polyol process, the green route consumes much less energy and is simpler. The aspect ratio of $1 \mathrm{D}$ Ag nanostructures is dependent on the temperature, $\mathrm{pH}$, solvent, and reagent type. The 1D Ag nanostructure formation mechanism, and how those factors can be tailored to increase their yield were discussed. The advantages of the green synthesis techniques make it a valid and promising platform for facile and high-yield synthesis of 1D Ag nanostructures such as AgNRs and AgNWs.

The current advances in in situ quantitative understanding using different instrumentation in the course of the reaction, including reduction, nucleation, and growth, will allow precise control of the size and morphology of metal nanostructures. In situ UV-vis can be used not only to investigate the optical properties of metal nanoparticles, but to monitor their quantitative formation and size as well. In situ FTIR spectroscopy can be used to find the different functional groups responsible for the reduction of metal ions and stabilization of metal nanoparticles from the peak positions in the spectrum. Future research is expected to focus on integration of these in situ characterization techniques to the batch green synthesis of 1D silver nanostructures to come up with the exact reaction mechanism, and consequently not only to control their size and morphology, but to expand the number of reducing and capping agents offered by nature for the synthesis of 1D silver nanostructures as well. Moreover, future research is expected to focus on large-scale industry relevant 1D silver nanostructure production in a green, sustainable, and continuous manner. The design of novel laboratory flow reactors has the potential to reduce waste, minimize the building space and energy requirements, and yield more accurate predictive models during development and manufacturing. The flow reactors, such as microfluidic reactors, offer uniform heat and mass transfer, more homogenous mixing of reagents, higher yield, and throughput, and are adaptable for in situ monitoring characterizations. However, the fabrication of microfluidic devices is time- and resource-intensive, and requires complicated facilities that support the advantages of millifluidic flow reactors in nanoparticle synthesis. These novel reactor systems offer similar advantages to microfluidic ones, while being easier to fabricate, simpler to reconstruct, and even more adaptable for in situ monitoring characterizations. A larger surface to volume ratio and precisely controlled flow patterns that consequently increase heat and mass transfer rate, coupled with inherent safety are among other advantages of flow millifluidic platforms $[66,387,388]$. The application of a continuous millifluidic reactor has the potential to be a method to overcome the challenges in batch green 1D silver nanostructure synthesis through control of a uniform chemical and thermal reaction environment in a small reaction volume. Novel in situ characterization techniques are essential for reaction mechanism investigation to control the morphology of the synthesized 1D Ag nanostructures in a continuous millifluidic reaction as well. In situ X-ray absorption spectroscopy (XAS) is a versatile technique that provides an opportunity to investigate the reaction dynamic and mechanism of 1D metal nanostructure growth in millifluidic reactor to further control their morphology, size distribution, and crystal structures. This expected future research direction will enable a synthesis route where 1D Ag nanostructure properties can be selected and tuned by simple alteration of reaction parameters and millifluidic reactor design to produce these nanostructures in an industrial relative scale in a green and sustainable manner. Furthermore, it is critical to optimize these green and sustainable techniques, not only in terms of scale-up capability, but also with respect to product quality and performance. Finally, the application of artificial neural network (ANN) tools in nanomaterial synthesis such as 1D silver nanostructures will save time and expenditure by predicting the reaction outcomes in which the most desirable nanomaterial size, morphology, and yield is achieved. Data from different characterization techniques including scanning electron microscopy (SEM), transmission electron microscopy (TEM), UV-visible spectroscopy (UV-vis), Fourier-transfer infrared spectroscopy (FTIR), and X-ray absorption spectroscopy (XAS) may be utilized to train the network to optimize the green synthesis of silver nanostructures as well as to predict the size and morphology of the synthesized silver nanostructures at different reaction conditions. It will consequently have a significant impact on industrial large-scale manufacturing of silver nanostructures, in which new molecule discovery as well as the development of alternatives to existing chemical processes will be discovered. Ultimately it will address the principal need in coupling green metal nanostructure synthesis, novel in situ characterization techniques, and artificial intelligence (AI) tools. A schematic on future direction in green and sustainable synthesis of $\mathrm{Ag}$ nanostructures with focus on 1D ones is shown in Figure 3. 


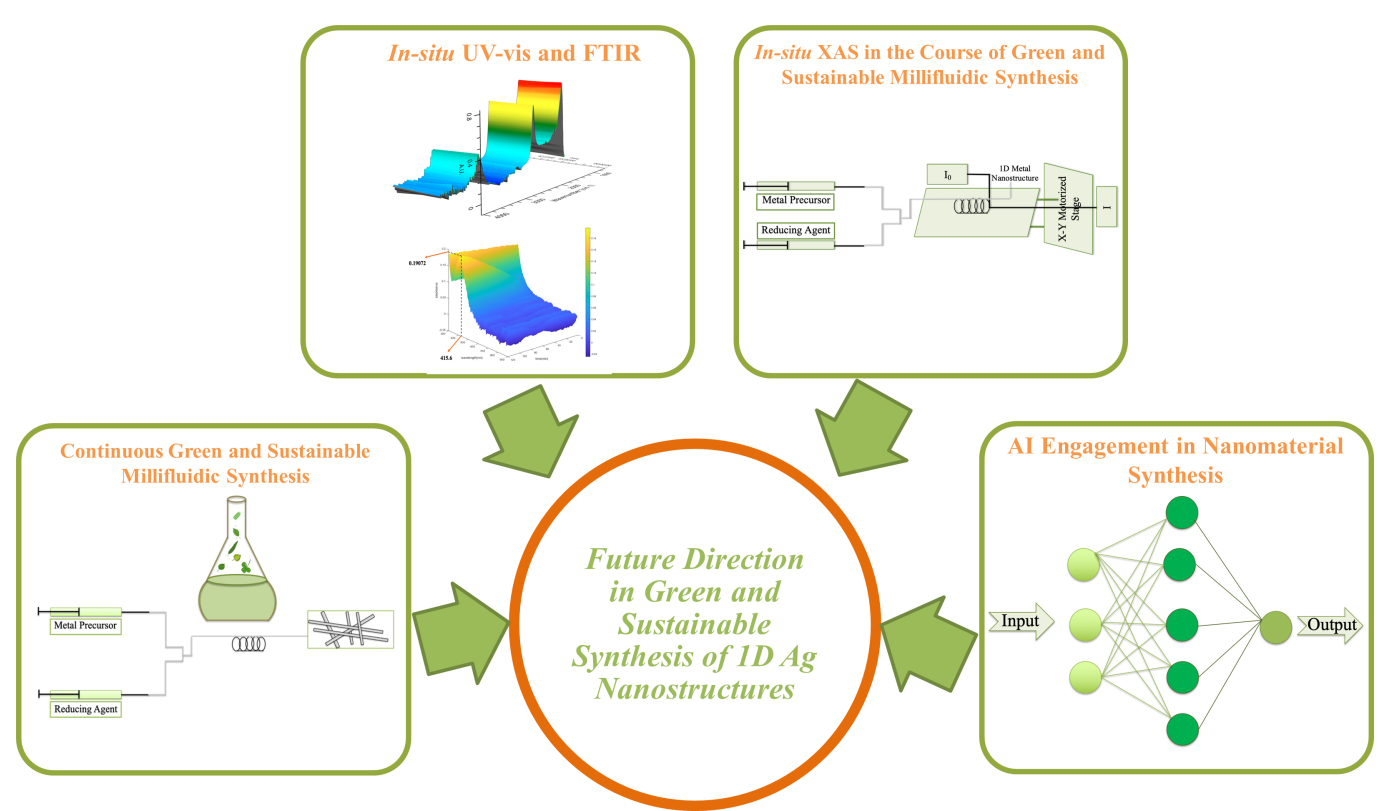

Figure 3: Expected future direction in green and sustainable synthesis of 1D Ag nanostructures.

\section{ORCID ${ }^{\circledR}$ iDs}

Sina Kaabipour - https://orcid.org/0000-0001-8879-0737 Shohreh Hemmati - https://orcid.org/0000-0003-0484-1133

\section{References}

1. Austin, L. A.; Mackey, M. A.; Dreaden, E. C.; El-Sayed, M. A. Arch. Toxicol. 2014, 88, 1391-1417. doi:10.1007/s00204-014-1245-3

2. Abadeer, N. S.; Murphy, C. J. J. Phys. Chem. C 2016, 120, 4691-4716. doi:10.1021/acs.jpcc.5b11232

3. Mirzaei, H.; Darroudi, M. Ceram. Int. 2017, 43, 907-914. doi:10.1016/j.ceramint.2016.10.051

4. Jahangirian, H.; Lemraski, E. G.; Webster, T. J.; Rafiee-Moghaddam, R.; Abdollahi, Y. Int. J. Nanomed. 2017, 12, 2957-2978. doi:10.2147/ijn.s127683

5. Wen, M. M.; El-Salamouni, N. S.; El-Refaie, W. M.; Hazzah, H. A.; Ali, M. M.; Tosi, G.; Farid, R. M.; Blanco-Prieto, M. J.; Billa, N.; Hanafy, A. S. J. Controlled Release 2017, 245, 95-107. doi:10.1016/j.jconrel.2016.11.025

6. Dos Santos Ramos, M. A.; Da Silva, P. B.; Spósito, L.; De Toledo, L. G.; Bonifácio, B. V.; Rodero, C. F.; Dos Santos, K. C.; Chorilli, M.; Bauab, T. M. Int. J. Nanomed. 2018, 13, 1179-1213. doi:10.2147/ijn.s146195

7. Chen, F.; Hableel, G.; Zhao, E. R.; Jokerst, J. V. J. Colloid Interface Sci. 2018, 521, 261-279. doi:10.1016/j.jcis.2018.02.053

8. Wilhelm, S.; Tavares, A. J.; Dai, Q.; Ohta, S.; Audet, J.; Dvorak, H. F.; Chan, W. C. W. Nat. Rev. Mater. 2016, 1, 16014. doi:10.1038/natrevmats.2016.14

9. Shi, J.; Kantoff, P. W.; Wooster, R.; Farokhzad, O. C. Nat. Rev. Cancer 2017, 17, 20-37. doi:10.1038/nrc.2016.108

10. Brownlee, W. J.; Seib, F. P. Sci. Rep. 2018, 8, 12318. doi:10.1038/s41598-018-30517-3
11. Cano, A. I.; Chiralt, A.; González-Martínez, C. Silver Composite Materials and Food Packaging. In Composite Materials for Food Packaging; Cirillo, G.; Kozlowski, M. A.; Spizzirri, U. G., Eds.; Wiley, 2018; pp 123-151. doi:10.1002/9781119160243.ch3

12. Kumar, S.; Shukla, A.; Baul, P. P.; Mitra, A.; Halder, D. Food Packag. Shelf Life 2018, 16, 178-184. doi:10.1016/j.fpsl.2018.03.008

13. Al-Shabib, N. A.; Husain, F. M.; Ahmed, F.; Khan, R. A.; Ahmad, I.; Alsharaeh, E.; Khan, M. S.; Hussain, A.; Rehman, M. T.; Yusuf, M.; Hassan, I.; Khan, J. M.; Ashraf, G. M.; Alsalme, A.; Al-Ajmi, M. F.; Tarasov, V. V.; Aliev, G. Sci. Rep. 2016, 6, 36761. doi:10.1038/srep36761

14. Wang, R.; Neoh, K. G.; Kang, E.-T.; Tambyah, P. A.; Chiong, E. J. Biomed. Mater. Res., Part B 2015, 103, 519-528. doi:10.1002/jbm.b.33230

15. Wu, K.; Yang, Y.; Zhang, Y.; Deng, J.; Lin, C. Int. J. Nanomed. 2015, 10, 7241-7252. doi:10.2147/ijn.s92307

16. Bhargava, A.; Pareek, V.; Roy Choudhury, S.; Panwar, J.; Karmakar, S. ACS Appl. Mater. Interfaces 2018, 10, 29325-29337. doi:10.1021/acsami.8b09475

17. van Hest, J. J. H. A.; Agronskaia, A. V.; Fokkema, J.; Montanarella, F.; Gregorio Puig, A.; de Mello Donega, C.; Meijerink, A.; Blab, G. A.; Gerritsen, H. C. J. Microsc. (Oxford, U. K.) 2019, 274, 13-22. doi:10.1111/jmi.12778

18. Varna, M.; Xuan, H. V.; Fort, E. Wiley Interdiscip. Rev.: Nanomed. Nanobiotechnol. 2018, 10, e1470. doi:10.1002/wnan.1470

19. Zhang, K.; Zhao, Q.; Qin, S.; Fu, Y.; Liu, R.; Zhi, J.; Shan, C. J. Colloid Interface Sci. 2019, 537, 316-324. doi:10.1016/j.jcis.2018.11.028

20. Sharma, R. K.; Nigam, S.; Chouryal, Y. N.; Nema, S.; Bera, S. P.; Bhargava, Y.; Ghosh, P. ACS Appl. Nano Mater. 2019, 2, 927-936. doi:10.1021/acsanm.8b02180 
21. Jafari, S.; Derakhshankhah, H.; Alaei, L.; Fattahi, A.; Varnamkhasti, B. S.; Sabouri, A. A. Biomed. Pharmacother. 2019, 109, 1100-1111. doi:10.1016/j.biopha.2018.10.167

22. Zhou, Z.; Yang, L.; Gao, J.; Chen, X. Adv. Mater. (Weinheim, Ger.) 2019, 31, 1804567. doi:10.1002/adma.201804567

23. Dash, S. P.; Patnaik, S. K.; Tripathy, S. K. Opt. Commun. 2019, 437, 388-391. doi:10.1016/j.optcom.2018.12.088

24. Liu, J.; Zhao, F.; Wang, H.; Zhang, W.; Hu, X.; Li, X.; Wang, Y. Opt. Mater. 2019, 89, 100-105. doi:10.1016/j.optmat.2018.12.055

25. Sharma, A.; Srivastava, A.; Jeon, Y.; Ahn, B. Metals (Basel, Switz.) 2018, 8, 347. doi:10.3390/met8050347

26. Wang, Y.; Liu, W.; Liu, W.; He, P.; Fan, Z.; Wang, X.; Yu, D.; Guo, J.; Guo, B.; Shen, H. Microelectron. Reliab. 2017, 78, 17-24. doi:10.1016/j.microrel.2017.07.069

27. Sochol, R. D.; Sweet, E.; Glick, C. C.; Wu, S.-Y.; Yang, C.; Restaino, M.; Lin, L. Microelectron. Eng. 2018, 189, 52-68. doi:10.1016/j.mee.2017.12.010

28. Lee, M.; Zenobi-Wong, M.; Chang, J. Nanocomposite materials comprising cationic nanoparticles and anionic polymers useful in methods for 3d printing thereof. U.S. Pat. Appl. US20190010288A1, Jan 10, 2019.

29. Fantino, E.; Chiappone, A.; Roppolo, I.; Manfredi, D.; Bongiovanni, R.; Pirri, C. F.; Calignano, F. Adv. Mater. (Weinheim, Ger.) 2016, 28 , 3712-3717. doi:10.1002/adma.201505109

30. Zhu, W.; Webster, T. J.; Zhang, L. G. Nanomedicine (London, U. K.) 2018, 13, 251-253. doi:10.2217/nnm-2017-0369

31. Tavakoli, M.; Malakooti, M. H.; Paisana, H.; Ohm, Y.; Green Marques, D.; Alhais Lopes, P.; Piedade, A. P.; de Almeida, A. T.; Majidi, C. Adv. Mater. (Weinheim, Ger.) 2018, 30 1801852. doi:10.1002/adma.201801852

32. Suresh, S.; Unni, G. E.; Satyanarayana, M.; Sreekumaran Nair, A.; Mahadevan Pillai, V. P. J. Colloid Interface Sci. 2018, 524, 236-244 doi:10.1016/j.jcis.2018.03.086

33. Wongrat, E.; Wongkrajang, S.; Chuejetton, A.; Bhoomanee, C.; Choopun, S. Mater. Res. Innovations 2019, 23, 66-72. doi:10.1080/14328917.2017.1376786

34. Xie, H.; Yin, X.; Chen, P.; Liu, J.; Yang, C.; Que, W.; Wang, G. Mater. Lett. 2019, 234, 311-314. doi:10.1016/j.matlet.2018.09.117

35. Li, H.; He, Y.; Wang, X.; Liu, D.; Liu, Z. J. Alloys Compd. 2019, 773, 743-751. doi:10.1016/j.jallcom.2018.09.279

36. Huang, S.; Wang, Y.; Shen, S.; Tang, Y.; Yu, A.; Kang, B.; Silva, S. R. P.; Lu, G. J. Colloid Interface Sci. 2019, 535, 308-317. doi:10.1016/j.jcis.2018.10.013

37. Zhao, F.; Chen, S.; Hu, Q.; Xue, G.; Ni, Q.; Jiang, Q.; Qiu, Y. Sep. Purif. Technol. 2017, 175, 130-139. doi:10.1016/j.seppur.2016.11.024

38. Morsi, R. E.; Alsabagh, A. M.; Nasr, S. A.; Zaki, M. M. Int. J. Biol. Macromol. 2017, 97, 264-269. doi:10.1016/j.ijbiomac.2017.01.032

39. Bindhu, M. R.; Umadevi, M. Spectrochim. Acta, Part A 2015, 135 , 373-378. doi:10.1016/j.saa.2014.07.045

40. Veisi, H.; Azizi, S.; Mohammadi, P. J. Cleaner Prod. 2018, 170 1536-1543. doi:10.1016/j.jclepro.2017.09.265

41. Choudhary, M. K.; Kataria, J.; Sharma, S. J. Cleaner Prod. 2018, 198, 882-890. doi:10.1016/j.jclepro.2018.09.015

42. Yan, Z.; Fu, L.; Zuo, X.; Yang, H. Appl. Catal., B 2018, 226, 23-30. doi:10.1016/j.apcatb.2017.12.040

43. Khoshnamvand, M.; Huo, C.; Liu, J. J. Mol. Struct. 2019, 1175, 90-96. doi:10.1016/j.molstruc.2018.07.089
44. Alexander, J. W. Surg. Infect. (New Rochelle, NY, U. S.) 2009, 10 289-292. doi:10.1089/sur.2008.9941

45. Srikar, S. K.; Giri, D. D.; Pal, D. B.; Mishra, P. K.; Upadhyay, S. N. Green Sustainable Chem. 2016, 6, 34-56. doi:10.4236/gsc.2016.61004

46. Wang, B.; Zhang, L.; Zhou, X. Spectrochim. Acta, Part A 2014, 121 63-69. doi:10.1016/j.saa.2013.10.013

47. Zhou, S.; Li, J.; Gilroy, K. D.; Tao, J.; Zhu, C.; Yang, X.; Sun, X.; Xia, Y. ACS Nano 2016, 10, 9861-9870. doi:10.1021/acsnano.6b05776

48. Khatoon, U. T.; Nageswara Rao, G. V. S.; Mohan, K. M.; Ramanaviciene, A.; Ramanavicius, A. Vacuum 2017, 146, 259-265. doi:10.1016/j.vacuum.2017.10.003

49. Khatami, M.; Zafarnia, N.; Heydarpoor Bami, M.; Sharifi, I.; Singh, H. J. Mycol. Med. 2018, 28, 637-644. doi:10.1016/j.mycmed.2018.07.007

50. Nair, A. K.; Sukumaran Nair, K. M.; Thomas, S.; Rouxel, D.; Alwarappan, S.; Kalarikkal, N. Langmuir 2018, 34, 13603-13614. doi:10.1021/acs.langmuir.8b02005

51. Wu, C.; Zhou, X.; Wei, J. Nanoscale Res. Lett. 2015, 10, 354 doi:10.1186/s11671-015-1058-1

52. Amirjani, A.; Koochak, N. N.; Haghshenas, D. F. Mater. Res. Express 2018, 6, 036204. doi:10.1088/2053-1591/aaf624

53. Debnath, B.; Das, R. Plasmonics 2019, 14, 1365-1375. doi:10.1007/s11468-019-00923-y

54. Haber, J.; Sokolov, K. Langmuir 2017, 33, 10525-10530. doi:10.1021/acs.langmuir.7b01362

55. Tsuji, M.; Gomi, S.; Maeda, Y.; Matsunaga, M.; Hikino, S.; Uto, K.; Tsuji, T.; Kawazumi, H. Langmuir 2012, 28, 8845-8861. doi:10.1021/la3001027

56. He, Y.; Wu, X.; Lu, G.; Shi, G. Mater. Chem. Phys. 2006, 98 , 178-182. doi:10.1016/j.matchemphys.2005.09.008

57. Jang, H.-S.; Seong, B.; Zang, X.; Lee, H.; Bae, J. W.; Cho, D.-H.; Kao, E.; Yang, C.; Kang, G.; Liu, Y.; Park, H. S.; Byun, D.; Lin, L. Adv. Mater. Interfaces 2018, 5, 1701491. doi:10.1002/admi.201701491

58. Ahn, H.-Y.; Cha, J.-R.; Gong, M.-S. Mater. Chem. Phys. 2015, 153 390-395. doi:10.1016/j.matchemphys.2015.01.030

59. Bashir, O.; Khan, Z. J. Mol. Liq. 2014, 199, 524-529. doi:10.1016/j.molliq.2014.09.041

60. Kim, B.-H.; Lee, J.-S. Mater. Chem. Phys. 2015, 149-150, 678-685. doi:10.1016/j.matchemphys.2014.11.026

61. Mohapatra, B.; Kuriakose, S.; Mohapatra, S. J. Alloys Compd. 2015 637, 119-126. doi:10.1016/j.jallcom.2015.02.206

62. Patarroyo, J.; Genç, A.; Arbiol, J.; Bastús, N. G.; Puntes, V. Chem. Commun. 2016, 52, 10960-10963. doi:10.1039/c6cc04796c

63. Zhang, Q.; Moran, C. H.; Xia, X.; Rycenga, M.; Li, N.; Xia, Y. Langmuir 2012, 28, 9047-9054. doi:10.1021/la300253a

64. Wiley, B. J.; Chen, Y.; McLellan, J. M.; Xiong, Y.; Li, Z.-Y.; Ginger, D.; Xia, Y. Nano Lett. 2007, 7, 1032-1036. doi:10.1021/nl070214f

65. Hemmati, S.; Barkey, D. P. ECS J. Solid State Sci. Technol. 2017, 6, P132-P137. doi:10.1149/2.0141704jss

66. Hemmati, S.; Barkey, D. P.; Eggleston, L.; Zukas, B.; Gupta, N.; Harris, M. ECS J. Solid State Sci. Technol. 2017, 6, P144-P149. doi:10.1149/2.0171704jss

67. Wang, H.; Wang, Y.; Chen, X. Colloids Surf., A 2019, 565, 154-161. doi:10.1016/j.colsurfa.2018.11.050

68. Khodashenas, B.; Ghorbani, H. R. Arabian J. Chem. 2015, 12 , 1823-1838. doi:10.1016/j.arabjc.2014.12.014 
69. Wei, L.; Lu, J.; Xu, H.; Patel, A.; Chen, Z.-S.; Chen, G. Drug Discovery Today 2015, 20, 595-601. doi:10.1016/j.drudis.2014.11.014

70. Bhakya, S.; Muthukrishnan, S.; Sukumaran, M.; Grijalva, M.; Cumbal, L.; Franklin Benjamin, J. H.; Senthil Kumar, T.; Rao, M. V. RSC Adv. 2016, 6, 81436-81446. doi:10.1039/c6ra17569d

71. Zheng, K.; Setyawati, M. I.; Leong, D. T.; Xie, J. Coord. Chem. Rev. 2018, 357, 1-17. doi:10.1016/j.ccr.2017.11.019

72. Ventola, C. L. Pharm. Ther. 2015, 40, 277-283.

73. Andersson, D. I.; Hughes, D. Nat. Rev. Microbiol. 2014, 12, 465-478. doi:10.1038/nrmicro3270

74. Besinis, A.; De Peralta, T.; Handy, R. D. Nanotoxicology 2014, 8 , 1-16. doi:10.3109/17435390.2012.742935

75. Seil, J. T.; Webster, T. J. Int. J. Nanomed. 2012, 7, 2767-2781. doi:10.2147/ijn.s24805

76. Morones, J. R.; Elechiguerra, J. L.; Camacho, A.; Holt, K.; Kouri, J. B.; Ramírez, J. T.; Yacaman, M. J. Nanotechnology 2005, 16, 2346-2353. doi:10.1088/0957-4484/16/10/059

77. Wang, F.; Yao, Y.; Zeng, X.; Huang, T.; Sun, R.; Xu, J.; Wong, C.-P. RSC Adv. 2016, 6, 41630-41636. doi:10.1039/c6ra00358c

78. Wang, F.; Zeng, X.; Yao, Y.; Sun, R.; Xu, J.; Wong, C.-P. Sci. Rep. 2016, 6, 19394. doi:10.1038/srep19394

79. Patole, A.; Lubineau, G. Carbon 2015, 81, 720-730. doi:10.1016/j.carbon.2014.10.014

80. Li, F.; Weir, M. D.; Chen, J.; Xu, H. H. K. Dent. Mater. 2013, 29, 450-461. doi:10.1016/j.dental.2013.01.012

81. Corrêa, J. M.; Mori, M.; Sanches, H. L.; da Cruz, A. D.; Poiate, E., Jr.; Poiate, I. A. V. P. Int. J. Biomater. 2015, 2015, 485275. doi: $10.1155 / 2015 / 485275$

82. Slane, J.; Vivanco, J.; Rose, W.; Ploeg, H.-L.; Squire, M. Mater. Sci. Eng., C 2015, 48, 188-196. doi:10.1016/j.msec.2014.11.068

83. Dadosh, T. Mater. Lett. 2009, 63, 2236-2238. doi:10.1016/j.matlet.2009.07.042

84. Jeong, S.-H.; Choi, H.; Kim, J. Y.; Lee, T.-W. Part. Part. Syst. Charact. 2015, 32, 164-175. doi:10.1002/ppsc.201400117

85. Qu, L.-L.; Geng, Y.-Y.; Bao, Z.-N.; Riaz, S.; Li, H. Microchim. Acta 2016, 183, 1307-1313. doi:10.1007/s00604-016-1760-4

86. Dissanayake, M. A. K. L.; Kumari, J. M. K. W.; Senadeera, G. K. R.; Thotawatthage, C. A. J. Appl. Electrochem. 2016, 46, 47-58. doi:10.1007/s10800-015-0886-0

87. Saravanan, S.; Kato, R.; Balamurugan, M.; Kaushik, S.; Soga, T. J. Sci.: Adv. Mater. Devices 2017, 2, 418-424. doi:10.1016/j.jsamd.2017.10.004

88. Meschi Amoli, B.; Trinidad, J.; Hu, A.; Zhou, Y. N.; Zhao, B. J. Mater. Sci.: Mater. Electron. 2014, 26, 590-600. doi:10.1007/s10854-014-2440-y

89. He, L.; Tjong, S. C. Mater. Sci. Eng., R 2016, 109, 1-101. doi:10.1016/j.mser.2016.08.002

90. Zhang, P.; Wyman, I.; Hu, J.; Lin, S.; Zhong, Z.; Tu, Y.; Huang, Z.; Wei, Y. Mater. Sci. Eng., B 2017, 223, 1-23. doi:10.1016/j.mseb.2017.05.002

91. Jiu, J.; Sugahara, T.; Nogi, M.; Nagao, S.; Suganuma, K. Silver nanowires transparent conductive films: Fabrication using different sintering techniques. In Proceedings of the IEEE Conference on Nanotechnology, 2013; pp 15-18. doi:10.1109/nano.2013.6720806

92. Bobinger, M.; Keddis, S.; Hinterleuthner, S.; Becherer, M.; Kluge, F.; Schwesinger, N.; Salmeron, J. F.; Lugli, P.; Rivadeneyra, A. IEEE Sens. J. 2019, 19, 1114-1126. doi:10.1109/jsen.2018.2879122
93. Youn, D.-Y.; Jung, U.; Naqi, M.; Choi, S.-J.; Lee, M.-G.; Lee, S.; Park, H.-J.; Kim, I.-D.; Kim, S. ACS Appl. Mater. Interfaces 2018, 10, 44678-44685. doi:10.1021/acsami.8b11928

94. De Guzman, N.; Ramos, M., Jr.; Balela, M. D. Mater. Res. Bull. 2018, 106, 446-454. doi:10.1016/j.materresbull.2018.06.030

95. Swartwout, R. Smoothing silver nanowires for optoelectronic applications, M.Sc Thesis, Massachusetts Institute of Technology, 2018.

96. Li, D.; Han, T.; Ruan, H. ACS Omega 2018, 3, 7191-7195. doi:10.1021/acsomega.8b01320

97. Ha, B.; Jo, S. Sci. Rep. 2017, 7, 11614. doi:10.1038/s41598-017-11964-w

98. Cheng, Y.; Wang, R.; Zhai, H.; Sun, J. Nanoscale 2017, 9, 3834-3842. doi:10.1039/c7nr00121e

99. Chebeleu, L. Using Nanowire Film in LCD Displays for Flexible Placement of Chip-on-Glass. Technical Disclosure Commons, 2017; https://www.tdcommons.org/dpubs_series/466.

100.Park, H.-G.; Heo, G.-S.; Park, S.-G.; Jeong, H.-C.; Lee, J. H.; Seo, D.-S. ECS Solid State Lett. 2015, 4, R50-R52. doi:10.1149/2.0031510ssl

101. Mallikarjuna, K.; Kim, H. ACS Appl. Mater. Interfaces 2019, 11, 1969-1978. doi:10.1021/acsami.8b14086

102.Wang, J.-L.; Lu, Y.-R.; Li, H.-H.; Liu, J.-W.; Yu, S.-H. J. Am. Chem. Soc. 2017, 139, 9921-9926. doi:10.1021/jacs.7b03227

103. Hemmati, S.; Barkey, D. P.; Gupta, N.; Banfield, R. ECS J. Solid State Sci. Technol. 2015, 4, P3075-P3079. doi:10.1149/2.0121504jss

104. Rafique, M.; Sadaf, I.; Rafique, M. S.; Tahir, M. B. Artif. Cells, Nanomed., Biotechnol. 2017, 45, 1272-1291. doi:10.1080/21691401.2016.1241792

105. Khayati, G. R.; Janghorban, K. Adv. Powder Technol. 2012, 23 , 808-813. doi:10.1016/j.apt.2011.11.001

106. Khayati, G. R.; Janghorban, K. Adv. Powder Technol. 2012, 23, 393-397. doi:10.1016/j.apt.2011.05.005

107. Jayaramudu, T.; Raghavendra, G. M.; Varaprasad, K.; Reddy, G. V. S.; Reddy, A. B.; Sudhakar, K.; Sadiku, E. R. J. Appl. Polym. Sci. 2016, 133, 43027. doi:10.1002/app.43027

108. Boutinguiza, M.; Comesaña, R.; Lusquiños, F.; Riveiro, A.; del Val, J.; Pou, J. Appl. Surf. Sci. 2015, 336, 108-111. doi:10.1016/j.apsusc.2014.09.193

109. Verma, S.; Rao, B. T.; Srivastava, A. P.; Srivastava, D.; Kaul, R.; Singh, B. Colloids Surf., A 2017, 527, 23-33. doi:10.1016/j.colsurfa.2017.05.003

110. Boutinguiza, M.; Fernández-Arias, M.; del Val, J.; Buxadera-Palomero, J.; Rodríguez, D.; Lusquiños, F.; Gil, F. J.; Pou, J. Mater. Lett. 2018, 231, 126-129. doi:10.1016/j.matlet.2018.07.134

111. Arboleda, D. M.; Santillán, J. M. J.; Arce, V. B.; Fernández van Raap, M. B.; Muraca, D.; Fernández, M. A.; Torres Sanchez, R. M.; Schinca, D. C.; Scaffardi, L. B. Mater. Charact. 2018, 140, 320-332. doi:10.1016/j.matchar.2018.04.021

112.Sportelli, M.; Izzi, M.; Volpe, A.; Clemente, M.; Picca, R.; Ancona, A.; Lugarà, P.; Palazzo, G.; Cioffi, N. Antibiotics (Basel, Switz.) 2018, 7, 67. doi:10.3390/antibiotics7030067

113.Simchi, A.; Ahmadi, R.; Reihani, S. M. S.; Mahdavi, A. Mater. Des. 2007, 28, 850-856. doi:10.1016/j.matdes.2005.10.017

114. Baker, C.; Pradhan, A.; Pakstis, L.; Pochan, D.; Shah, S. I. J. Nanosci. Nanotechnol. 2005, 5, 244-249. doi:10.1166/jnn.2005.034 
115. Malekzadeh, M.; Halali, M. Chem. Eng. J. 2011, 168, 441-445. doi:10.1016/j.cej.2010.12.081

116. Hui, K. S.; Hui, K. N.; Dinh, D. A.; Tsang, C. H.; Cho, Y. R.; Zhou, W.; Hong, X.; Chun, H.-H. Acta Mater. 2014, 64, 326-332. doi:10.1016/j.actamat.2013.10.045

117.Park, M.; Sohn, Y.; Shin, W. G.; Lee, J.; Ko, S. H. Ultrason. Sonochem. 2015, 22, 35-40. doi:10.1016/j.ultsonch.2014.05.007

118. Raghavendra, G. M.; Jung, J.; Kim, D.; Varaprasad, K.; Seo, J. Carbohydr. Polym. 2016, 152, 558-565. doi:10.1016/j.carbpol.2016.07.045

119.Jung, J.; Raghavendra, G. M.; Kim, D.; Seo, J. Int. J. Biol. Macromol. 2018, 107, 2285-2290. doi:10.1016/j.jibiomac.2017.10.108

120.Javey, A.; Dai, H. J. Am. Chem. Soc. 2005, 127, 11942-11943. doi:10.1021/ja0536668

121.Wu, J.; Zan, X.; Li, S.; Liu, Y.; Cui, C.; Zou, B.; Zhang, W.; Xu, H.; Duan, H.; Tian, D.; Huang, W.; Huo, F. Nanoscale 2014, 6, 749-752. doi:10.1039/c3nr05033e

122.Shih, S.-J.; Chien, I.-C. Powder Technol. 2013, 237, 436-441. doi:10.1016/j.powtec.2012.12.032

123.Jang, H. D.; Kim, S. K.; Chang, H.; Jo, E. H.; Roh, K. M.; Choi, J.-H.; Choi, J.-W. Aerosol Sci. Technol. 2015, 49, 538-546. doi:10.1080/02786826.2015.1050086

124.Keskar, M.; Sabatini, C.; Cheng, C.; Swihart, M. T. Nanoscale Adv. 2019, 1, 627-635. doi:10.1039/c8na00281a

125. Ramnani, S. P.; Biswal, J.; Sabharwal, S. Radiat. Phys. Chem. 2007, 76, 1290-1294. doi:10.1016/j.radphyschem.2007.02.074

126.Juby, K. A.; Dwivedi, C.; Kumar, M.; Kota, S.; Misra, H. S.; Bajaj, P. N. Carbohydr. Polym. 2012, 89, 906-913. doi:10.1016/j.carbpol.2012.04.033

127. Biswal, J.; Misra, N.; Borde, L. C.; Sabharwal, S. Radiat. Phys. Chem. 2013, 83, 67-73. doi:10.1016/j.radphyschem.2012.10.003

128. Uttayarat, P.; Eamsiri, J.; Tangthong, T.; Suwanmala, P. Adv. Mater. Sci. Eng. 2015, 376082. doi:10.1155/2015/376082

129. Ashkarran, A. A. Curr. Appl. Phys. 2010, 10, 1442-1447. doi:10.1016/j.cap.2010.05.010

130. Kumar, P.; Singh, P. K.; Hussain, M.; Kumar Das, A. Adv. Sci. Lett. 2016, 22, 3-7. doi:10.1166/asl.2016.6772

131.Zhang, H.; Zou, G.; Liu, L.; Tong, H.; Li, Y.; Bai, H.; Wu, A. J. Mater. Sci. 2017, 52, 3375-3387. doi:10.1007/s10853-016-0626-9

132. Tseng, K.-H.; Chou, C.-J.; Liu, T.-C.; Tien, D.-C.; Wu, T.-C.; Stobinski, L. Adv. Mater. Sci. Eng. 2018, 3240959. doi:10.1155/2018/3240959

133. Tseng, K.-H.; Chou, C.-J.; Liu, T.-C.; Tien, D.-C.; Chang, C. Y.; Stobinski, L. Nanotechnol. Rev. 2018, 7, 1-9. doi:10.1515/ntrev-2017-0167

134.Liu, J.; Zhang, X.; Yu, M.; Li, S.; Zhang, J. Small 2012, 8, 310-316. doi:10.1002/smll.201101423

135. Manikprabhu, D.; Lingappa, K. J. J. Pharm. Res. (Mohali, India) 2013, 6, 255-260. doi:10.1016/j.jopr.2013.01.022

136.Zhang, J. D.; Yu, M.; Liu, J. H.; Li, S. M.; Meng, Y. B. Mater. Sci. Forum 2016, 847, 194-199. doi:10.4028/www.scientific.net/msf.847.194

137. Natsuki, J.; Natsuki, T.; Hashimoto, Y. Int. J. Mater. Sci. Appl. 2015, 4, 325-332. doi:10.11648/j.jmsa.20150405.17

138. Thanh, N. T. K.; Maclean, N.; Mahiddine, S. Chem. Rev. 2014, 114, 7610-7630. doi:10.1021/cr400544s

139.Lee, J.; Yang, J.; Kwon, S. G.; Hyeon, T. Nat. Rev. Mater. 2016, 1, 16034. doi:10.1038/natrevmats.2016.34
140.Khan, M.; Shaik, M. R.; Adil, S. F.; Khan, S. T.; Al-Warthan, A.; Siddiqui, M. R. H.; Tahir, M. N.; Tremel, W. Dalton Trans. 2018, 47, 11988-12010. doi:10.1039/c8dt01152d

141. Mourdikoudis, S.; Liz-Marzán, L. M. Chem. Mater. 2013, 25 , 1465-1476. doi:10.1021/cm4000476

142. Montes-García, V.; Pérez-Juste, J.; Pastoriza-Santos, I.; Liz-Marzán, L. M. Chem. - Eur. J. 2014, 20, 10874-10883. doi:10.1002/chem.201403107

143. Jadalannagari, S.; Deshmukh, K.; Ramanan, S. R.; Kowshik, M. Appl. Nanosci. 2014, 4, 133-141. doi:10.1007/s13204-013-0197-x

144. Ueno, S.; Nakashima, K.; Sakamoto, Y.; Wada, S. Nanomaterials 2015, 5, 386-397. doi:10.3390/nano5020386

145. Arun Kumar, K. V.; John, J.; Sooraj, T. R.; Raj, S. A.; Unnikrishnan, N. V.; Selvaraj, N. B. Appl. Surf. Sci. 2019, 472, 40-45. doi:10.1016/j.apsusc.2018.05.178

146. Li, X.; Kim, N.; Youn, S.; An, T. K.; Kim, J.; Lim, S.; Kim, S. H. Polymers (Basel, Switz.) 2019, 11, 158. doi:10.3390/polym11010158

147.Lee, S. J.; Heo, M.; Lee, D.; Han, S.; Moon, J. H.; Lim, H. N.; Kwon, I. K. Appl. Surf. Sci. 2018, 432, 317-323. doi:10.1016/j.apsusc.2017.04.030

148. Wang, Y.; Zheng, Y.; Huang, C. Z.; Xia, Y. J. Am. Chem. Soc. 2013, 135, 1941-1951. doi:10.1021/ja311503q

149. Dugandžić, V.; Hidi, I. J.; Weber, K.; Cialla-May, D.; Popp, J. Anal. Chim. Acta 2016, 946, 73-79. doi:10.1016/j.aca.2016.10.018

150.Zhu, J.; Xu, X.; Liu, J.; Zheng, Y.; Hou, S. RSC Adv. 2015, 5 , 74126-74131. doi:10.1039/c5ra13884a

151. Raza, M.; Kanwal, Z.; Rauf, A.; Sabri, A.; Riaz, S.; Naseem, S. Nanomaterials 2016, 6, 74. doi:10.3390/nano6040074

152. Singha, D.; Barman, N.; Sahu, K. J. Colloid Interface Sci. 2014, 413 , 37-42. doi:10.1016/j.jcis.2013.09.009

153. Matsuhisa, N.; Inoue, D.; Zalar, P.; Jin, H.; Matsuba, Y.; Itoh, A.; Yokota, T.; Hashizume, D.; Someya, T. Nat. Mater. 2017, 16, 834-840. doi:10.1038/nmat4904

154. Yang, J.; Li, Y.; Jiang, B.; Fu, Y. J. Nanophotonics 2018, 12, 036008. doi:10.1117/1.jnp.12.036008

155. Ciobanu, C. S.; Iconaru, S. L.; Chifiriuc, M. C.; Costescu, A.; Le Coustumer, P.; Predoi, D. BioMed Res. Int. 2013, 916218. doi:10.1155/2013/916218

156. Bahlawane, N.; Premkumar, P. A.; Brechling, A.; Reiss, G.; Kohse-Höinghaus, K. Chem. Vap. Deposition 2007, 13, 401-407. doi:10.1002/cvde.200706610

157. Kuzminova, A.; Beranová, J.; Polonskyi, O.; Shelemin, A.; Kylián, O.; Choukourov, A.; Slavínská, D.; Biederman, H. Surf. Coat. Technol. 2016, 294, 225-230. doi:10.1016/j.surfcoat.2016.03.097

158.Zhang, K.-X.; Wen, X.; Yao, C.-B.; Li, J.; Zhang, M.; Li, Q.-H.; Sun, W.-J.; Wu, J.-D. Chem. Phys. Lett. 2018, 698, 147-151. doi:10.1016/j.cplett.2018.03.018

159.Wani, I. A.; Khatoon, S.; Ganguly, A.; Ahmed, J.; Ganguli, A. K.; Ahmad, T. Mater. Res. Bull. 2010, 45, 1033-1038. doi:10.1016/j.materresbull.2010.03.028

160. Chen, D.; Qiao, X.; Qiu, X.; Chen, J.; Jiang, R. J. Mater. Sci.: Mater. Electron. 2011, 22, 6-13. doi:10.1007/s10854-010-0074-2

161.Du, D.; Yang, X.; Yang, Y.; Zhao, Y.; Wang, Y. Micromachines 2019, 10, 42. doi:10.3390/mi10010042

162. Guzmán, M. G.; Dille, J.; Godet, S. Int. J. Chem. Biomol. Eng. 2009, 2, 104-111.

163. Khaydarov, R. A.; Khaydarov, R. R.; Gapurova, O.; Estrin, Y.; Scheper, T. J. Nanopart. Res. 2009, 11, 1193-1200. doi:10.1007/s11051-008-9513-x 
164. Reicha, F. M.; Sarhan, A.; Abdel-Hamid, M. I.; El-Sherbiny, I. M. Carbohydr. Polym. 2012, 89, 236-244.

doi:10.1016/j.carbpol.2012.03.002

165. Nasretdinova, G. R.; Fazleeva, R. R.; Mukhitova, R. K.; Nizameev, I. R.; Kadirov, M. K.; Ziganshina, A. Y.; Yanilkin, V. V. Electrochem. Commun. 2015, 50, 69-72. doi:10.1016/j.elecom.2014.11.016

166. Chitsazi, M. R.; Korbekandi, H.; Asghari, G.; Bahri Najafi, R.; Badii, A.; Iravani, S. Artif. Cells, Nanomed., Biotechnol. 2016, 44, 328-333. doi:10.3109/21691401.2014.949726

167.Divya, M.; Kiran, G. S.; Hassan, S.; Selvin, J. Biocatal. Agric. Biotechnol. 2019, 18, 101037. doi:10.1016/j.bcab.2019.101037

168.Saravanan, M.; Barik, S. K.; MubarakAli, D.; Prakash, P.; Pugazhendhi, A. Microb. Pathog. 2018, 116, 221-226. doi:10.1016/j.micpath.2018.01.038

169.Saravanan, C.; Rajesh, R.; Kaviarasan, T.; Muthukumar, K.; Kavitake, D.; Shetty, P. H. Biotechnol. Rep. 2017, 15, 33-40. doi:10.1016/j.btre.2017.02.006

170. Rajora, N.; Kaushik, S.; Jyoti, A.; Kothari, S. L. IET Nanobiotechnol. 2016, 10, 367-373. doi:10.1049/iet-nbt.2015.0107

171.Srivastava, S.; Bhargava, A.; Pathak, N.; Srivastava, P. Microb. Pathog. 2019, 129, 136-145. doi:10.1016/j.micpath.2019.02.013

172. Khalil, N. M.; Abd El-Ghany, M. N.; Rodríguez-Couto, S. Chemosphere 2019, 218, 477-486. doi:10.1016/j.chemosphere.2018.11.129

173.Sanguiñedo, P.; Fratila, R. M.; Estevez, M. B.; de la Fuente, J. M.; Grazú, V.; Alborés, S. Nano Biomed. Eng. 2018, 10, 165-173. doi:10.5101/nbe.v10i2.p165-173

174.Ma, L.; Su, W.; Liu, J.-X.; Zeng, X.-X.; Huang, Z.; Li, W.; Liu, Z.-C.; Tang, J.-X. Mater. Sci. Eng., C 2017, 77, 963-971. doi:10.1016/j.msec.2017.03.294

175.Bayram, S.; Zahr, O.; Del Re, J.; Blum, A. S. TMV Disk Scaffolds for Making sub-30 nm Silver Nanorings. In Protein Scaffolds; Udit, A., Ed.; Methods in Molecular Biology, Vol. 1798; Humana Press: New York, 2018; pp 109-118. doi:10.1007/978-1-4939-7893-9_9

176. Yang, C.; Jung, S.; Yi, H. Biochem. Eng. J. 2014, 89, 10-20. doi:10.1016/j.bej.2013.12.008

177.Daphne, J.; Francis, A.; Mohanty, R.; Ojha, N.; Das, N. Res. J. Pharm. Technol. 2018, 11, 83-92. doi:10.5958/0974-360x.2018.00016.1

178. Fernández, J. G.; Fernández-Baldo, M. A.; Berni, E.; Camí, G.; Durán, N.; Raba, J.; Sanz, M. I. Process Biochem. (Oxford, U. K.) 2016, 51, 1306-1313. doi:10.1016/j.procbio.2016.05.021

179.Korbekandi, H.; Mohseni, S.; Mardani Jouneghani, R.; Pourhossein, M.; Iravani, S. Artif. Cells, Nanomed., Biotechnol. 2016, 44, 235-239. doi:10.3109/21691401.2014.937870

180. Kumar, V.; Yadav, S. K. J. Chem. Technol. Biotechnol. 2009, 84, 151-157. doi:10.1002/jctb.2023

181. Iravani, S. Green Chem. 2011, 13, 2638-2650. doi:10.1039/c1gc15386b

182.Mashwani, Z.-u.-R.; Khan, T.; Khan, M. A.; Nadhman, A. Appl. Microbiol. Biotechnol. 2015, 99, 9923-9934. doi:10.1007/s00253-015-6987-1

183. Rajeshkumar, S.; Bharath, L. V. Chem.-Biol. Interact. 2017, 273, 219-227. doi:10.1016/j.cbi.2017.06.019

184.Sri Ramkumar, S. R.; Sivakumar, N.; Selvakumar, G.; Selvankumar, T.; Sudhakar, C.; Ashokkumar, B.; Karthi, S. RSC Adv. 2017, 7, 34548-34555. doi:10.1039/c6ra28328d
185. Ahmed, S.; Ahmad, M.; Swami, B. L.; Ikram, S. J. Adv. Res. 2016, 7, 17-28. doi:10.1016/j.jare.2015.02.007

186. Shaik, M. R.; Khan, M.; Kuniyil, M.; Al-Warthan, A.; Alkhathlan, H. Z.; Siddiqui, M. R. H.; Shaik, J. P.; Ahamed, A.; Mahmood, A.; Khan, M.; Adil, S. F. Sustainability 2018, 10, 913. doi:10.3390/su10040913

187. Behravan, M.; Hossein Panahi, A.; Naghizadeh, A.; Ziaee, M.; Mahdavi, R.; Mirzapour, A. Int. J. Biol. Macromol. 2019, 124, 148-154. doi:10.1016/j.jjbiomac.2018.11.101

188. Ranoszek-Soliwoda, K.; Tomaszewska, E.; Małek, K.; Celichowski, G.; Orlowski, P.; Krzyzowska, M.; Grobelny, J. Colloids Surf., B 2019, 177, 19-24. doi:10.1016/j.colsurfb.2019.01.037

189.Dahoumane, S. A.; Mechouet, M.; Wijesekera, K.; Filipe, C. D. M.; Sicard, C.; Bazylinski, D. A.; Jeffryes, C. Green Chem. 2017, 19, 552-587. doi:10.1039/c6gc02346k

190. Dahoumane, S.; Jeffryes, C.; Mechouet, M.; Agathos, S. Bioengineering 2017, 4, 14. doi:10.3390/bioengineering4010014

191.Dahoumane, S. A.; Mechouet, M.; Alvarez, F. J.; Agathos, S. N.; Jeffryes, C. Bionatura 2016, 1, 196-201. doi:10.21931/rb/2016.01.04.7

192. Rahman, A.; Kumar, S.; Bafana, A.; Dahoumane, S. A.; Jeffryes, C. Molecules 2019, 24, 98. doi:10.3390/molecules24010098

193. Abdel-Raouf, N.; Al-Enazi, N. M.; Ibraheem, I. B. M.; Alharbi, R. M.; Alkhulaifi, M. M. Saudi J. Biol. Sci. 2018, 26, 1207-1215. doi:10.1016/j.sjbs.2018.01.007

194.Talekar, S.; Joshi, G.; Chougle, R.; Nainegali, B.; Desai, S.; Joshi, A.; Kambale, S.; Kamat, P.; Haripurkar, R.; Jadhav, S.; Nadar, S. Catal. Commun. 2014, 53, 62-66. doi:10.1016/j.catcom.2014.05.003

195. Talekar, S.; Joshi, A.; Chougle, R.; Nakhe, A.; Bhojwani, R. Nano-Struct. Nano-Objects 2016, 6, 23-33. doi:10.1016/j.nanoso.2016.03.002

196. Mukherjee, K.; Gupta, R.; Kumar, G.; Kumari, S.; Biswas, S.; Padmanabhan, P. J. Genet. Eng. Biotechnol. 2018, 16, 527-536. doi:10.1016/j.jgeb.2018.04.004

197.Philip, D. Spectrochim. Acta, Part A 2010, 75, 1078-1081. doi:10.1016/j.saa.2009.12.058

198. Anuradha, K.; Bangal, P.; Madhavendra, S. S. Macromol. Res. 2016, 24, 152-162. doi:10.1007/s13233-016-4018-4

199. Hemmati, S.; Retzlaff-Roberts, E.; Scott, C.; Harris, M. T. J. Nanomater. 2019, 9641860. doi:10.1155/2019/9641860

200. Meshram, S. M.; Bonde, S. R.; Gupta, I. R.; Gade, A. K.; Rai, M. K. IET Nanobiotechnol. 2013, 7, 28-32. doi:10.1049/iet-nbt.2012.0002

201. May, B. M. M.; Oluwafemi, O. S. Int. J. Electrochem. Sci. 2016, 11, 8096-8108. doi:10.20964/2016.09.29

202. Nadagouda, M. N.; Varma, R. S. Green Chem. 2006, 8, 516-518. doi:10.1039/b601271j

203. Malassis, L.; Dreyfus, R.; Murphy, R. J.; Hough, L. A.; Donnio, B.; Murray, C. B. RSC Adv. 2016, 6, 33092-33100. doi:10.1039/c6ra00194g

204. Nadagouda, M. N.; Varma, R. S. J. Nanomater. 2008, 2008, 782358. doi:10.1155/2008/782358

205. Akhavan, O. Carbon 2015, 81, 158-166. doi:10.1016/j.carbon.2014.09.044

206. Granata, G.; Yamaoka, T.; Pagnanelli, F.; Fuwa, A. J. Nanopart. Res. 2016, 18, 133. doi:10.1007/s11051-016-3438-6

207.Lee, Y.-I.; Kwon, Y.-T.; Kim, S.; Lee, K.-J.; Choa, Y.-H. Thin Solid Films 2016, 616, 260-264. doi:10.1016/j.tsf.2016.08.032

208. Guzman, M.; Dille, J.; Godet, S. Nanomedicine (N. Y., NY, U. S.) 2012, 8, 37-45. doi:10.1016/j.nano.2011.05.007

209.Szczepanowicz, K.; Stefańska, J.; Socha, R. P.; Warszyński, P. Physicochem. Probl. Miner. Process. 2010, 45, 85-98. 
210. Chumachenko, V.; Kutsevol, N.; Rawiso, M.; Schmutz, M.; Blanck, C. Nanoscale Res. Lett. 2014, 9, 164. doi:10.1186/1556-276x-9-164

211. Gurusamy, V.; Krishnamoorthy, R.; Gopal, B.; Veeraravagan, V.; Neelamegam, P. Inorg. Nano-Met. Chem. 2017, 47, 761-767. doi:10.1080/15533174.2015.1137074

212.Dai, L.; Nadeau, B.; An, X.; Cheng, D.; Long, Z.; Ni, Y. Sci. Rep. 2016, 6, 36497. doi:10.1038/srep36497

213.Zamiri, R.; Azmi, B. Z.; Sadrolhosseini, A. R.; Ahangar, H. A.; Zaidan, A. W.; Mahdi, M. A. Int. J. Nanomed. 2011, 6, 71-75. doi:10.2147/ijn.s14005

214. Iravani, S.; Korbekandi, H.; Mirmohammadi, S. V.; Zolfaghari, B. Res. Pharm. Sci. 2014, 9, 385-406.

215. Mostafa, A. A.; Sayed, S. R. M.; Solkamy, E. N.; Khan, M.; Shaik, M. R.; Al-Warthan, A.; Adil, S. F. J. Nanomater. 2015, 789178. doi:10.1155/2015/789178

216.Siddiqi, K. S.; Husen, A.; Rao, R. A. K. J. Nanobiotechnol. 2018, 16 , 14. doi:10.1186/s12951-018-0334-5

217. Mousavi, S. M.; Hashemi, S. A.; Ghasemi, Y.; Atapour, A.; Amani, A. M.; Savar Dashtaki, A.; Babapoor, A.; Arjmand, O. Artif. Cells, Nanomed., Biotechnol. 2018, 46, s855-s872. doi:10.1080/21691401.2018.1517769

218.Jorge de Souza, T. A.; Rosa Souza, L. R.; Franchi, L. P. Ecotoxicol. Environ. Saf. 2019, 171, 691-700. doi:10.1016/j.ecoenv.2018.12.095

219. Mittal, A. K.; Chisti, Y.; Banerjee, U. C. Biotechnol. Adv. 2013, 31 , 346-356. doi:10.1016/j.biotechadv.2013.01.003

220.Xing, T.; Sunarso, J.; Yang, W.; Yin, Y.; Glushenkov, A. M.; Li, L. H.; Howlett, P. C.; Chen, Y. Nanoscale 2013, 5, 7970-7976. doi:10.1039/c3nr02328a

221. Khayati, G. R.; Janghorban, K. Trans. Nonferrous Met. Soc. China 2013, 23, 1520-1524. doi:10.1016/s1003-6326(13)62625-4

222.Ullah, M.; Ali, M. E.; Hamid, S. B. A. Rev. Adv. Mater. Sci. 2014, 37, $1-14$.

223.Cui, B. Z.; Zheng, L. Y.; Waryoba, D.; Marinescu, M.; Hadjipanayis, G. C. J. Appl. Phys. 2011, 109, 07 A728. doi:10.1063/1.3562447

224.Raffi, M.; Rumaiz, A. K.; Hasan, M. M.; Shah, S. I. J. Mater. Res. 2007, 22, 3378-3384. doi:10.1557/jmr.2007.0420

225. Kruis, F. E.; Fissan, H.; Rellinghaus, B. Mater. Sci. Eng., B 2000, 69-70, 329-334. doi:10.1016/s0921-5107(99)00298-6

226. Magnusson, M. H.; Deppert, K.; Malm, J.-O.; Bovin, J.-O.; Samuelson, L. J. Nanopart. Res. 1999, 1, 243-251. doi:10.1023/a:1010012802415

227.Tien, D.-C.; Tseng, K.-H.; Liao, C.-Y.; Huang, J.-C.; Tsung, T.-T. J. Alloys Compd. 2008, 463, 408-411. doi:10.1016/j.jallcom.2007.09.048

228. Amendola, V.; Meneghetti, M. Phys. Chem. Chem. Phys. 2009, 11, 3805-3821. doi:10.1039/b900654k

229. Amendola, V.; Meneghetti, M. Phys. Chem. Chem. Phys. 2013, 15, 3027-3046. doi:10.1039/c2cp42895d

230.Ghaffarian, H. R.; Saiedi, M.; Sayadnejad, M. A.; Rashidi, A. M. Iran. J. Chem. Chem. Eng. 2011, 30, 1-6.

231. Iravani, S. Methods for Preparation of Metal Nanoparticles. In Metal Nanoparticles: Synthesis and Applications in Pharmaceutical Sciences; Thota, S.; Crans, D. C., Eds.; Wiley: Weinheim, Germany, 2017; Vol. 63, pp 15-31. doi:10.1002/9783527807093.ch2

232. Niederberger, M. Acc. Chem. Res. 2007, 40, 793-800. doi:10.1021/ar600035e
233. Muromachi, T.; Tsujino, T.; Kamitani, K.; Maeda, K. J. Sol-Gel Sci. Technol. 2006, 40, 267-272. doi:10.1007/s10971-006-8386-7

234. Milea, C. A.; Bogatu, C.; Duță, A. Bull. Transilvania Univ. Brasov, Ser. I 2011, 4, 59-66.

235. Noritomi, H.; Umezawa, Y.; Miyagawa, S.; Kato, S. Adv. Chem. Eng. Sci. 2011, 1, 299-304. doi:10.4236/aces.2011.14041

236. Setua, P.; Ghatak, C.; Rao, V. G.; Das, S. K.; Sarkar, N. J. Phys. Chem. B 2012, 116, 3704-3712. doi:10.1021/jp203043k

237.Zhang, W.; Qiao, X.; Chen, J. Colloids Surf., A 2007, 299, 22-28. doi:10.1016/j.colsurfa.2006.11.012

238. Setua, P.; Pramanik, R.; Sarkar, S.; Seth, D.; Sarkar, N. J. Phys. Chem. B 2009, 113, 5677-5680. doi:10.1021/jp810229m

239. Egorova, E. M.; Revina, A. A. Colloids Surf., A 2000, 168, 87-96. doi:10.1016/s0927-7757(99)00513-0

240.Solanki, J. N.; Murthy, Z. V. P. Ind. Eng. Chem. Res. 2011, 50, 7338-7344. doi:10.1021/ie200536q

241.Piszczek, P.; Radtke, A. Silver Nanoparticles Fabricated Using Chemical Vapor Deposition and Atomic Layer Deposition Techniques: Properties, Applications and Perspectives: Review. In Noble and Precious Metals; Seehra, M. S.; Bristow, A. D., Eds.; IntechOpen: London, U.K., 2018; pp 187-213. doi:10.5772/intechopen.71571

242. Goswami, S.; Aich, K.; Das, S.; Basu Roy, S.; Pakhira, B.; Sarkar, S. RSC Adv. 2014, 4, 14210-14214. doi:10.1039/c3ra46663a

243. Mahapatra, A. K.; Karmakar, P.; Manna, S.; Maiti, K.; Mandal, D. J. Photochem. Photobiol., A 2017, 334, 1-12. doi:10.1016/j.jphotochem.2016.10.032

244.EPA, Risk Information System Division. Chemical Assessment Summary of Hydrazine/Hydrazine sulfate. https://cfpub.epa.gov/ncea/iris2/chemicalLanding.cfm?substance_nmb $r=352$

245. EPA, Risk Information System Division. Chemical Assessment Summary of $N, N$-Dimethylformamide. https://cfpub.epa.gov/ncea/iris2/chemicalLanding.cfm?substance_nmb $r=511$.

246. New Jersey Department of Health and Senior Services. Hazardous substance fact sheet on sodium borohydride. 1999.

247. Titkov, A. I.; Gerasimov, E. Y.; Shashkov, M. V.; Logutenko, O. A.; Bulina, N. V.; Yukhin, Y. M.; Lyakhov, N. Z. Colloid J. 2016, 78, 515-524. doi:10.1134/s1061933×16040189

248. Kim, D.; Jeong, S.; Moon, J. Nanotechnology 2006, 17, 4019-4024. doi:10.1088/0957-4484/17/16/004

249.Zhao, T.; Sun, R.; Yu, S.; Zhang, Z.; Zhou, L.; Huang, H.; Du, R. Colloids Surf., A 2010, 366, 197-202. doi:10.1016/j.colsurfa.2010.06.005

250.Chen, C.; Wang, L.; Yu, H.; Jiang, G.; Yang, Q.; Zhou, J.; Xiang, W.; Zhang, J. Mater. Chem. Phys. 2008, 107, 13-17. doi:10.1016/j.matchemphys.2007.06.048

251.Parveen, K.; Banse, V.; Ledwani, L. AIP Conf. Proc. 2016, 1724 , 020048. doi:10.1063/1.4945168

252.Sharma, V. K.; Yngard, R. A.; Lin, Y. Adv. Colloid Interface Sci. 2009, 145, 83-96. doi:10.1016/j.cis.2008.09.002

253. Hussain, I.; Singh, N. B.; Singh, A.; Singh, H.; Singh, S. C. Biotechnol. Lett. 2016, 38, 545-560. doi:10.1007/s10529-015-2026-7

254.Chen, J.; Wang, J.; Zhang, X.; Jin, Y. Mater. Chem. Phys. 2008, 108, 421-424. doi:10.1016/j.matchemphys.2007.10.019

255. Francis, S.; Joseph, S.; Koshy, E. P.; Mathew, B. Artif. Cells, Nanomed., Biotechnol. 2018, 46, 795-804. doi:10.1080/21691401.2017.1345921 
256. Long, D.; Wu, G.; Chen, S. Radiat. Phys. Chem. 2007, 76, 1126-1131. doi:10.1016/j.radphyschem.2006.11.001

257.Raghavendra, G. M.; Jayaramudu, T.; Varaprasad, K.; Sadiku, R.; Ray, S. S.; Mohana Raju, K. Carbohydr. Polym. 2013, 93, 553-560. doi:10.1016/j.carbpol.2012.12.035

258. Raghavendra, G. M.; Jayaramudu, T.; Varaprasad, K.; Mohan Reddy, G. S.; Raju, K. M. RSC Adv. 2015, 5, 14351-14358. doi:10.1039/c4ra15995k

259.Shivaji, S.; Madhu, S.; Singh, S. Process Biochem. (Oxford, U. K.) 2011, 46, 1800-1807. doi:10.1016/j.procbio.2011.06.008

260.Sastry, M.; Ahmad, A.; Islam Khan, M.; Kumar, R. Curr. Sci. 2003, 85, 162-170.

261.Dujardin, E.; Peet, C.; Stubbs, G.; Culver, J. N.; Mann, S. Nano Lett. 2003, 3, 413-417. doi:10.1021/nl034004o

262.Lee, S.-Y.; Royston, E.; Culver, J. N.; Harris, M. T. Nanotechnology 2005, 16, S435-S441. doi:10.1088/0957-4484/16/7/019

263. Thangavelu, R. M.; Ganapathy, R.; Ramasamy, P.; Krishnan, K. Arabian J. Chem. 2020, 13, 2750-2765. doi:10.1016/j.arabjc.2018.07.006

264.Sathishkumar, R. S.; Sundaramanickam, A.; Srinath, R.; Ramesh, T.; Saranya, K.; Meena, M.; Surya, P. J. Saudi Chem. Soc. 2019, 23, 1180-1191. doi:10.1016/j.jscs.2019.07.008

265.Dahoumane, S. A.; Wujcik, E. K.; Jeffryes, C Enzyme Microb. Technol. 2016, 95, 13-27. doi:10.1016/j.enzmictec.2016.06.008

266. Naghdi, M.; Taheran, M.; Brar, S. K.; Verma, M.; Surampalli, R. Y.; Valero, J. R. Beilstein J. Nanotechnol. 2015, 6, 2354-2376. doi:10.3762/bjnano.6.243

267. Kalimuthu, K.; Suresh Babu, R.; Venkataraman, D.; Bilal, M.; Gurunathan, S. Colloids Surf., B 2008, 65, 150-153. doi:10.1016/j.colsurfb.2008.02.018

268.Shahverdi, A. R.; Minaeian, S.; Shahverdi, H. R.; Jamalifar, H.; Nohi, A.-A. Process Biochem. (Oxford, U. K.) 2007, 42, 919-923. doi:10.1016/j.procbio.2007.02.005

269. Lateef, A.; Adelere, I. A.; Gueguim-Kana, E. B.; Asafa, T. B.; Beukes, L. S. Int. Nano Lett. 2015, 5, 29-35. doi:10.1007/s40089-014-0133-4

270.Ahmad, A.; Mukherjee, P.; Senapati, S.; Mandal, D.; Khan, M. I.; Kumar, R.; Sastry, M. Colloids Surf., B 2003, 28, 313-318. doi:10.1016/s0927-7765(02)00174-1

271.Naqvi, S. Z.; Kiran, U.; Ali, M. I.; Jamal, A.; Hameed, A.; Ahmed, S.; Ali, N. Int. J. Nanomed. 2013, 8, 3187-3195. doi:10.2147/ijn.s49284

272. Young, M.; Debbie, W.; Uchida, M.; Douglas, T. Annu. Rev. Phytopathol. 2008, 46, 361-384. doi:10.1146/annurev.phyto.032508.131939

273.Adigun, O. O.; Retzlaff-Roberts, E. L.; Novikova, G.; Wang, L.; Kim, B.-S.; llavsky, J.; Miller, J. T.; Loesch-Fries, L. S.; Harris, M. T. Langmuir 2017, 33, 1716-1724. doi:10.1021/acs.langmuir.6b03341

274.Lee, K. Z.; Basnayake Pussepitiyalage, V.; Lee, Y.-H.; Loesch-Fries, L. S.; Harris, M. T.; Hemmati, S.; Solomon, K. V. Biotechnol. J. 2020, 2000311. doi:10.1002/biot.202000311

275.da Silva Ferreira, V.; ConzFerreira, M. E.; Lima, L. M. T. R.; Frasés, S.; de Souza, W.; Sant'Anna, C. Enzyme Microb. Technol. 2017, 97, 114-121. doi:10.1016/j.enzmictec.2016.10.018

276. Monteiro, C. M.; Castro, P. M. L.; Malcata, F. X. Biotechnol. Prog. 2012, 28, 299-311. doi:10.1002/btpr.1504

277.Barwal, I.; Ranjan, P.; Kateriya, S.; Yadav, S. C. J. Nanobiotechnol. 2011, 9, 56. doi:10.1186/1477-3155-9-56

278.Jena, J.; Pradhan, N.; Prasad Dash, B.; Behari Sukla, L.; Panda, P. Int. J. Nanomater. Biostructures 2013, 3, 1-8.
279. Muthusamy, G.; Thangasamy, S.; Raja, M.; Chinnappan, S.; Kandasamy, S. Environ. Sci. Pollut. Res. 2017, 24, 19459-19464. doi:10.1007/s11356-017-9772-0

280.Galvez, A. M.; Ramos, K. M.; Teja, A. J.; Baculi, R. J. Microbiol., Biotechnol. Food Sci. 2019, 2019, 970-978. doi:10.15414/jmbfs.2019.8.4.970-978

281. Muthulakshmi, K.; Uma, C.; Sivagurunathan, P.; Yoganathan, K.; Satheeshkumar, S. J. Pharmacogn. Phytochem. 2018, 7, 741-747.

282. Wang, C.; Kim, Y. J.; Singh, P.; Mathiyalagan, R.; Jin, Y.; Yang, D. C. Artif. Cells, Nanomed., Biotechnol. 2016, 44, 1127-1132. doi:10.3109/21691401.2015.1011805

283. Gurunathan, S.; Kalishwaralal, K.; Vaidyanathan, R.; Venkataraman, D.; Pandian, S. R. K.; Muniyandi, J.; Hariharan, N.; Eom, S. H. Colloids Surf., B 2009, 74, 328-335. doi:10.1016/j.colsurfb.2009.07.048

284. Nanda, A.; Saravanan, M. Nanomedicine (N. Y., NY, U. S.) 2009, 5, 452-456. doi:10.1016/j.nano.2009.01.012

285. Husain, S.; Afreen, S.; Hemlata; Yasin, D.; Afzal, B.; Fatma, T. J. Microbiol. Methods 2019, 162, 77-82. doi:10.1016/j.mimet.2019.05.011

286. Rolim, W. R.; Pelegrino, M. T.; de Araújo Lima, B.; Ferraz, L. S.; Costa, F. N.; Bernardes, J. S.; Rodigues, T.; Brocchi, M.; Seabra, A. B. Appl. Surf. Sci. 2019, 463, 66-74. doi:10.1016/j.apsusc.2018.08.203

287. Flores-González, M.; Talavera-Rojas, M.; Soriano-Vargas, E.; Rodríguez-González, V. New J. Chem. 2018, 42, 2133-2139. doi:10.1039/c7nj03812g

288. Kumar, B.; Smita, K.; Cumbal, L.; Debut, A. Saudi J. Biol. Sci. 2017, 24, 45-50. doi:10.1016/j.sjbs.2015.09.006

289. Dhand, V.; Soumya, L.; Bharadwaj, S.; Chakra, S.; Bhatt, D.; Sreedhar, B. Mater. Sci. Eng., C 2016, 58, 36-43. doi:10.1016/j.msec.2015.08.018

290. Hu, S.; Hsieh, Y.-L. Int. J. Biol. Macromol. 2016, 82, 856-862. doi:10.1016/j.ijbiomac.2015.09.066

291. Dong, L. Micro Nano Lett. 2019, 14, 137-141. doi:10.1049/mnl.2018.5110

292.Jeevika, A.; Ravi Shankaran, D. J. Colloid Interface Sci. 2015, 458, 155-159. doi:10.1016/j.jcis.2015.07.045

293. Nthunya, L. N.; Derese, S.; Gutierrez, L.; Verliefde, A. R.; Mamba, B. B.; Barnard, T. G.; Mhlanga, S. D. New J. Chem. 2019, 43, 4168-4180. doi:10.1039/c8nj06160b

294. Ahmed, S.; Saifullah; Ahmad, M.; Swami, B. L.; Ikram, S. J. Radiat. Res. Appl. Sci. 2016, 9, 1-7. doi:10.1016/j.jrras.2015.06.006

295. Anjum, S.; Jacob, G.; Gupta, B. Emergent Mater. 2019, 2, 113-122. doi:10.1007/s42247-019-00023-x

296. Ibrahim, H. M. M. J. Radiat. Res. Appl. Sci. 2015, 8, 265-275. doi:10.1016/j.jrras.2015.01.007

297. Alsammarraie, F. K.; Wang, W.; Zhou, P.; Mustapha, A.; Lin, M. Colloids Surf., B 2018, 171, 398-405. doi:10.1016/j.colsurfb.2018.07.059

298. Padalia, H.; Moteriya, P.; Chanda, S. Arabian J. Chem. 2015, 8 , 732-741. doi:10.1016/j.arabjc.2014.11.015

299.Kumar, B.; Smita, K.; Cumbal, L.; Debut, A.; Pathak, R. N. Bioinorg. Chem. Appl. 2014, 2014, 784268. doi:10.1155/2014/784268 300.El-Refai, A. A.; Ghoniem, G. A.; El-Khateeb, A. Y.; Hassaan, M. M. J. Nanostruct. Chem. 2018, 8, 71-81. doi:10.1007/s40097-018-0255-8 301. Qin, Y.; Ji, X.; Jing, J.; Liu, H.; Wu, H.; Yang, W. Colloids Surf., A 2010, 372, 172-176. doi:10.1016/j.colsurfa.2010.10.013 
302.Han, C.; Nagendra, V.; Baig, R. B. N.; Varma, R. S.; Nadagouda, M. N. Appl. Sci. 2015, 5, 415-426. doi:10.3390/app5030415

303.AbdelRahim, K.; Mahmoud, S. Y.; Ali, A. M.; Almaary, K. S.; Mustafa, A. E. Z. M. A.; Husseiny, S. M. Saudi J. Biol. Sci. 2017, 24, 208-216. doi:10.1016/j.sjbs.2016.02.025

304.Amooaghaie, R.; Saeri, M. R.; Azizi, M. Ecotoxicol. Environ. Saf. 2015, 120, 400-408. doi:10.1016/j.ecoenv.2015.06.025

305.Komes, D.; Belščak-Cvitanović, A.; Horžić, D.; Rusak, G.; Likić, S.; Berendika, M. Phytochem. Anal. 2011, 22, 172-180. doi:10.1002/pca.1264

306. Rice-evans, C. A.; Miller, N. J.; Bolwell, P. G.; Bramley, P. M.; Pridham, J. B. Free Radical Res. 1995, 22, 375-383. doi:10.3109/10715769509145649

307.Khan, M.; Khan, M.; Adil, S. F.; Tahir, M. N.; Tremel, W.; Alkhathlan, H. Z.; Al-Warthan, A.; Siddiqui, M. R. H. Int. J. Nanomed. 2013, 8, 1507-1516. doi:10.2147/ijn.s43309

308.Tangahu, B. V.; Sheikh Abdullah, S. R.; Basri, H.; Idris, M.; Anuar, N.; Mukhlisin, M. Int. J. Chem. Eng. 2011, 2011, 939161. doi:10.1155/2011/939161

309.Ali, H.; Khan, E.; Sajad, M. A. Chemosphere 2013, 91, 869-881. doi:10.1016/j.chemosphere.2013.01.075

310.Callahan, D. L.; Hare, D. J.; Bishop, D. P.; Doble, P. A.; Roessner, U. RSC Adv. 2016, 6, 2337-2344. doi:10.1039/c5ra23953b

311.de Oliveira, L. M.; Ma, L. Q.; Santos, J. A. G.; Guilherme, L. R. G.; Lessl, J. T. Environ. Pollut. 2014, 184, 187-192. doi:10.1016/j.envpol.2013.08.025

312.Ma, Y.; Oliveira, R. S.; Nai, F.; Rajkumar, M.; Luo, Y.; Rocha, I.; Freitas, H. J. Environ. Manage. 2015, 156, 62-69. doi:10.1016/j.jenvman.2015.03.024

313.Antoniadis, V.; Levizou, E.; Shaheen, S. M.; Ok, Y. S.; Sebastian, A.; Baum, C.; Prasad, M. N. V.; Wenzel, W. W.; Rinklebe, J. Earth-Sci. Rev. 2017, 171, 621-645 doi:10.1016/j.earscirev.2017.06.005

314.Gardea-Torresdey, J. L.; Gomez, E.; Peralta-Videa, J. R.; Parsons, J. G.; Troiani, H.; Jose-Yacaman, M. Langmuir 2003, 19, 1357-1361. doi:10.1021/la020835i

315. Marchiol, L. Ital. J. Agron. 2012, 7, e37. doi:10.4081/ija.2012.e37

316. Haverkamp, R. G.; Marshall, A. T.; Van Agterveld, D. J. Nanopart. Res. 2007, 9, 697-700. doi:10.1007/s11051-006-9198-y

317. Harris, A. T.; Bali, R. J. Nanopart. Res. 2008, 10, 691-695. doi:10.1007/s11051-007-9288-5

318. Haverkamp, R. G.; Marshall, A. T. J. Nanopart. Res. 2009, 11, 1453-1463. doi:10.1007/s11051-008-9533-6

319. Beattie, I. R.; Haverkamp, R. G. Metallomics 2011, 3, 628-632. doi:10.1039/c1mt00044f

320.Park, Y.; Hong, Y. N.; Weyers, A.; Kim, Y. S.; Linhardt, R. J. IET Nanobiotechnol. 2011, 5, 69-78. doi:10.1049/iet-nbt.2010.0033

321. Ratan, Z. A.; Haidere, M. F.; Nurunnabi, M.; Shahriar, S. M.; Ahammad, A. J. S.; Shim, Y. Y.; Reaney, M. J. T.; Cho, J. Y. Cancers 2020, 12, 855. doi:10.3390/cancers12040855

322. Makarov, V. V.; Love, A. J.; Sinitsyna, O. V.; Makarova, S. S.; Yaminsky, I. V.; Taliansky, M. E.; Kalinina, N. O. ActaNaturae 2014, 6, 35-44. doi:10.32607/20758251-2014-6-1-35-44

323. Mukunthan, K. S.; Balaji, S. Int. J. Green Nanotechnol. 2012, 4, 71-79. doi:10.1080/19430892.2012.676900

324. Antonysamy Johnson, M.; Shibila, T.; Amutha, S.; Menezes, I. R. A.; da Costa, J. G. M.; Sampaio, N. F. L.; Coutinho, H. D. M. Pharmaceuticals 2020, 13, 66. doi:10.3390/ph13040066
325.Sivakumar, M.; Surendar, S.; Jayakumar, M.; Seedevi, P.; Sivasankar, P.; Ravikumar, M.; Anbazhagan, M.; Murugan, T.; Siddiqui, S. S.; Loganathan, S. J. Cluster Sci. 2020, 1-11. doi:10.1007/s10876-020-01775-x

326. Nouri, A.; Tavakkoli Yaraki, M.; Lajevardi, A.; Rezaei, Z.; Ghorbanpour, M.; Tanzifi, M. Colloid Interface Sci. Commun. 2020, 35, 100252. doi:10.1016/j.colcom.2020.100252

327. Tanase, C.; Berta, L.; Mare, A.; Man, A.; Talmaciu, A. I.; Roșca, I.; Mircia, E.; Volf, I.; Popa, V. I. Eur. J. Wood Wood Prod. 2020, 78, 281-291. doi:10.1007/s00107-020-01502-3

328. Mohaghegh, S.; Osouli-Bostanabad, K.; Nazemiyeh, H.; Javadzadeh, Y.; Parvizpur, A.; Barzegar-Jalali, M.; Adibkia, K. Adv. Powder Technol. 2020, 31, 1169-1180. doi:10.1016/j.apt.2019.12.039

329. Ranoszek-Soliwoda, K.; Tomaszewska, E.; Socha, E.; Krzyczmonik, P.; Ignaczak, A.; Orlowski, P.; Krzyzowska, M.; Celichowski, G.; Grobelny, J. J. Nanopart. Res. 2017, 19, 273. doi:10.1007/s11051-017-3973-9

330. Phongtongpasuk, S.; Norasingsatorn, T.; Yongvanich, N. Key Eng. Mater. 2019, 824, 149-155. doi:10.4028/www.scientific.net/kem.824.149

331.Singh, A.; Gaud, B.; Jaybhaye, S. Mater. Sci. Energy Technol. 2020, 3, 232-236. doi:10.1016/j.mset.2019.08.004

332. Handayani, W.; Ningrum, A. S.; Imawan, C. J. Phys.: Conf. Ser. 2020, 1428, 012021. doi:10.1088/1742-6596/1428/1/012021

333.Dada, A. O.; Adekola, F. A.; Adeyemi, O. S.; Bello, O. M.; Oluwaseun, A. C.; Awakan, O. J.; Grace, F.-A. A. Exploring the Effect of Operational Factors and Characterization Imperative to the Synthesis of Silver Nanoparticles. In Silver Nanoparticles:Fabrication, Characterization and Applications; Maaz, K., Ed.; IntechOpen: London, U.K., 2018; pp 165-184. doi:10.5772/intechopen.76947

334.Ghodake, G. S.; Deshpande, N. G.; Lee, Y. P.; Jin, E. S. Colloids Surf., B 2010, 75, 584-589. doi:10.1016/j.colsurfb.2009.09.040

335. Raut, R. W.; Mendhulkar, V. D.; Kashid, S. B. J. Photochem. Photobiol., B 2014, 132, 45-55. doi:10.1016/j.jphotobiol.2014.02.001

336. Kanipandian, N.; Thirumurugan, R. Ind. Crops Prod. 2014, 55, 1-10. doi:10.1016/j.indcrop.2014.01.042

337.Singh, M.; Sinha, I.; Mandal, R. K. Mater. Lett. 2009, 63, 425-427. doi:10.1016/j.matlet.2008.10.067

338. Madivoli, E. S.; Kareru, P. G.; Gachanja, A. N.; Mugo, S. M.; Makhanu, D. S.; Wanakai, S. I.; Gavamukulya, Y. J. Inorg. Organomet. Polym. Mater. 2020, 30, 2842-2850. doi:10.1007/s10904-019-01432-5

339. Anbu, P.; Gopinath, S. C. B.; Yun, H. S.; Lee, C.-G. J. Mol. Struct. 2019, 1177, 302-309. doi:10.1016/j.molstruc.2018.09.075

340.Sun, Q.; Cai, X.; Li, J.; Zheng, M.; Chen, Z.; Yu, C.-P. Colloids Surf., A 2014, 444, 226-231. doi:10.1016/j.colsurfa.2013.12.065

341.Cruz, D.; Falé, P. L.; Mourato, A.; Vaz, P. D.; Luisa Serralheiro, M.; Lino, A. R. L. Colloids Surf., B 2010, 81, 67-73. doi:10.1016/j.colsurfb.2010.06.025

342.Jiang, X. C.; Chen, W. M.; Chen, C. Y.; Xiong, S. X.; Yu, A. B. Nanoscale Res. Lett. 2010, 6, 32. doi:10.1007/s11671-010-9780-1

343. Liu, H.; Zhang, H.; Wang, J.; Wei, J. Arabian J. Chem. 2020, 13, 1011-1019. doi:10.1016/j.arabjc.2017.09.004

344. Hasnain, M. S.; Javed, M. N.; Alam, M. S.; Rishishwar, P.; Rishishwar, S.; Ali, S.; Nayak, A. K.; Beg, S. Mater. Sci. Eng., C 2019, 99, 1105-1114. doi:10.1016/j.msec.2019.02.061 
345. Htwe, Y. Z. N.; Chow, W. S.; Suda, Y.; Mariatti, M. Mater. Today: Proc. 2019, 17, 568-573. doi:10.1016/j.matpr.2019.06.336

346. Khan, S.; Singh, S.; Gaikwad, S.; Nawani, N.; Junnarkar, M.; Pawar, S. V. Environ. Sci. Pollut. Res. 2019, 27, 27221-27233. doi:10.1007/s11356-019-05239-2

347.Parashar, V.; Parashar, R.; Sharma, B.; Pandey, A. C. Dig. J. Nanomater. Biostructures 2009, 4, 45-50.

348. Kowalska, D.; Krajnik, B.; Olejnik, M.; Twardowska, M.; Czechowski, N.; Hofmann, E.; MacKowski, S. Sci. World J. 2013, 2013, 670412. doi:10.1155/2013/670412

349.Ćwik, M.; Buczyńska, D.; Sulowska, K.; Roźniecka, E.; Mackowski, S.; Niedziółka-Jönsson, J. Materials 2019, 12, 721. doi:10.3390/ma12050721

350.Kim, S.; Yun, T. G.; Kang, C.; Son, M.-J.; Kang, J.-G.; Kim, I.-H.; Lee, H.-J.; An, C.-H.; Hwang, B. Mater. Des. 2018, 151, $1-7$. doi:10.1016/j.matdes.2018.04.047

351.Yu, C. H.; Chi, H. H.; Hsiao, C. C. SID Symp. Dig. Tech. Pap. 2019, 50, 476-478. doi:10.1002/sdtp.13533

352.Dai, H.; Spaid, M. SID Symp. Dig. Tech. Pap. 2018, 49, 397-400. doi:10.1002/sdtp.12583

353. Yi, P.; Zhu, Y.; Deng, Y. Fabrication and Applications of Flexible Transparent Electrodes Based on Silver Nanowires. In Flexible Electronics; Rackauskas, S., Ed.; Intechopen, 2018. doi:10.5772/intechopen.77506

354.Zhu, Y.; Deng, Y.; Yi, P.; Peng, L.; Lai, X.; Lin, Z. Adv. Mater. Technol. (Weinheim, Ger.) 2019, 4, 1900413. doi:10.1002/admt.201900413

355. Li, B.; Ye, S.; Stewart, I. E.; Alvarez, S.; Wiley, B. J. Nano Lett. 2015, 15, 6722-6726. doi:10.1021/acs.nanolett.5b02582

356. Du, Y.; Shi, L.; He, T.; Sun, X.; Mo, Y. Appl. Surf. Sci. 2008, 255 , 1901-1905. doi:10.1016/j.apsusc.2008.06.140

357.Bergin, S. M.; Chen, Y.-H.; Rathmell, A. R.; Charbonneau, P.; Li, Z.-Y.; Wiley, B. J. Nanoscale 2012, 4, 1996-2004. doi:10.1039/c2nr30126a

358. Hemmati, S.; Barkey, D. P.; Gupta, N. J. Nanopart. Res. 2016, 18, 249. doi:10.1007/s11051-016-3561-4

359.Govindaraj, A.; Satishkumar, B. C.; Nath, M.; Rao, C. N. R. Chem. Mater. 2000, 12, 202-205. doi:10.1021/cm990546o

360.Zhang, D.; Qi, L.; Ma, J.; Cheng, H. Chem. Mater. 2001, 13, 2753-2755. doi:10.1021/cm0105007

361.Junaidi; Yunus, M.; Harsojo; Suharyadi, E.; Triyana, K. Int. J. Adv. Sci., Eng. Inf. Technol. 2016, 6, 365-369. doi:10.18517/ijaseit.6.3.808

362.Fahad, S.; Yu, H.; Wang, L.; Zain-ul-Abdin; Haroon, M.; Ullah, R. S.; Nazir, A.; Naveed, K.-u.-R.; Elshaarani, T.; Khan, A. J. Mater. Sci. 2019, 54, 997-1035. doi:10.1007/s10853-018-2994-9

363.Sun, Y.; Gates, B.; Mayers, B.; Xia, Y. Nano Lett. 2002, 2, 165-168. doi:10.1021/nl010093y

364.Sun, Y.; Xia, Y. Adv. Mater. (Weinheim, Ger.) 2002, 14, 833-837. doi:10.1002/1521-4095(20020605)14:11<833::aid-adma833>3.0.co;2$\mathrm{k}$

365. Wiley, B.; Sun, Y.; Xia, Y. Langmuir 2005, 21, 8077-8080. doi:10.1021/la050887i

366. Korte, K. E.; Skrabalak, S. E.; Xia, Y. J. Mater. Chem. 2008, 18, 437-441. doi:10.1039/b714072j

367. Hemmati, S.; Harris, M. T.; Barkey, D. P. J. Nanomater. 2020, 2020, 9341983. doi:10.1155/2020/9341983

368. Tian, X.; Li, J.; Pan, S. J. Nanopart. Res. 2009, 11, 1839-1844. doi:10.1007/s11051-009-9700-4
369. Barnaby, S. N.; Yu, S. M.; Fath, K. R.; Tsiola, A.; Khalpari, O. Banerjee, I. A. Nanotechnology 2011, 22, 225605. doi:10.1088/0957-4484/22/22/225605

370.Lin, L.; Wang, W.; Huang, J.; Li, Q.; Sun, D.; Yang, X.; Wang, H.; He, N.; Wang, Y. Chem. Eng. J. 2010, 162, 852-858. doi:10.1016/j.cej.2010.06.023

371.Soleimani, F. F.; Saleh, T.; Shojaosadati, S. A.; Poursalehi, R. Bionanosci. 2018, 8, 72-80. doi:10.1007/s12668-017-0423-1

372.Peng, H.-C.; Park, J.; Zhang, L.; Xia, Y. J. Am. Chem. Soc. 2015, 137, 6643-6652. doi:10.1021/jacs.5b03040

373.Huo, D.; Kim, M. J.; Lyu, Z.; Shi, Y.; Wiley, B. J.; Xia, Y. Chem. Rev. 2019, 119, 8972-9073. doi:10.1021/acs.chemrev.8b00745

374. Bulut, E.; Ozacar, M. Ind. Eng. Chem. Res. 2009, 48, 5686-5690. doi:10.1021/ie801779f

375.Yi, Z.; Li, X.; Xu, X.; Luo, B.; Luo, J.; Wu, W.; Yi, Y.; Tang, Y. Colloids Surf., A 2011, 392, 131-136. doi:10.1016/j.colsurfa.2011.09.045

376. Tian, X.; Wang, W.; Cao, G. Mater. Lett. 2007, 61, 130-133. doi:10.1016/j.matlet.2006.04.021

377.Cruz, B. H.; Díaz-Cruz, J. M.; Ariño, C.; Esteban, M. Electroanalysis 2000, 12, 1130-1137.

doi:10.1002/1521-4109(200010)12:14<1130::aid-elan1130>3.0.co;2-7

378. Ahmad, T. J. Nanotechnol. 2014, 2014, 954206. doi:10.1155/2014/954206

379. Martínez-Castañón, G. A.; Niño-Martínez, N.; Martínez-Gutierrez, F.; Martínez-Mendoza, J. R.; Ruiz, F. J. Nanopart. Res. 2008, 10 , 1343-1348. doi:10.1007/s11051-008-9428-6

380.Liu, J.; Qin, G.; Raveendran, P.; Ikushima, Y. Chem. - Eur. J. 2007, 12, 2131-2138. doi:10.1002/chem.200500925

381.Schuette, W. M.; Buhro, W. E. Chem. Mater. 2014, 26, 6410-6417. doi: $10.1021 / \mathrm{cm} 502827 \mathrm{~b}$

382. Villalpando, M.; Saavedra-Molina, A.; Rosas, G. Mater. Sci. Eng., C 2020, 114, 110973. doi:10.1016/j.msec.2020.110973

383. Tiwari, P.; Joshi, A.; Dubey, B. K. Asian J. Pharm. Educ. Res. 2017, 6, 45-53.

384. Chen, J.; Herricks, T.; Geissler, M.; Xia, Y. J. Am. Chem. Soc. 2004, 126, 10854-10855. doi:10.1021/ja0468224

385. You, H.; Fang, J. Nano Today 2016, 11, 145-167. doi:10.1016/j.nantod.2016.04.003

386.Jang, H.-W.; Hwang, B.-Y.; Lee, K.-W.; Kim, Y.-M.; Kim, J.-Y. AIP Adv. 2018, 8, 025303. doi:10.1063/1.5011263

387.Dong, B.; Hadinoto, K. Int. J. Pharm. 2017, 523, 42-51. doi:10.1016/j.ijpharm.2017.03.021

388. Seaberg, J.; Kaabipour, S.; Hemmati, S.; Ramsey, J. D. Eur. J. Pharm. Biopharm. 2020, 154, 127-135. doi:10.1016/j.ejpb.2020.07.006 


\section{License and Terms}

This is an Open Access article under the terms of the Creative Commons Attribution License (https://creativecommons.org/licenses/by/4.0). Please note that the reuse, redistribution and reproduction in particular requires that the author(s) and source are credited and that individual graphics may be subject to special legal provisions.

The license is subject to the Beilstein Journal of Nanotechnology terms and conditions: (https://www.beilstein-journals.org/bjnano/terms)

The definitive version of this article is the electronic one which can be found at:

https://doi.org/10.3762/bjnano.12.9 